\section{Collaboration interprofessionnelle}

CO 01

\section{Pratiques simulées: apprentissage procédural d'étudiants en médecine encadrés par d'autres étudiants en santé. Quel ressenti ?}

Evelaine LOUIS ${ }^{1}$ Christine AMMIRATI ${ }^{2}$, Carole AMSALLEM ${ }^{3}$, Béatrice JAMAULT ${ }^{4}$, Catherine QUEMENER ${ }^{3}$, Claire FAUVET $^{5}$, Maxime GIGNON ${ }^{6}$

${ }^{1}$ SimUSanté ${ }^{\odot}$, UFR médecine Amiens, Université PicardieJules-Verne, Amiens, France

${ }^{2}$ SimUSanté ${ }^{\oplus}$, Département de médecine d'urgence, CHU Amiens, centre de recherche en psychologie: cognition, psychisme et organisations, Université Picardie-Jules-Verne, Amiens, France

${ }^{3}$ SimUSanté ${ }^{\odot}$, Centre d'enseignement des soins d'urgence, Département de médecine d'urgence, $\mathrm{CHU}$, Amiens, France

${ }^{4}$ SimUSanté ${ }^{\odot}$, Institut de formation des manipulateurs en électroradiologie, $\mathrm{CHU}$, Amiens, France

${ }^{5}$ SimUSanté ${ }^{\oplus}$, UFR médecine, Université Picardie-Jules-Verne, Amiens, France

6 SimUSanté $^{\odot}$, santé des populations, CHU, Amiens, Laboratoire éducation et pratiques en santé, Paris XIII, France

Contexte : L'objectif de SimUSanté, espace pédagogique hospitalouniversitaire étant d' "« apprendre ensemble pour soigner ensemble», nous avons souhaité faire encadrer des étudiants en médecine par des étudiants d'autres professions paramédicales dans le cadre de simulations procédurales

Objectif: L'objectif de notre étude est d'évaluer le ressenti des étudiants-tuteurs paramédicaux et tutorés étudiants en médecine (EM). L'objectif secondaire est d'évaluer le retentissement de ces temps partagés sur la connaissance des métiers paramédicaux par les EM.

Méthode: L'étude est menée à partir de l'optionnel facultaire «Pratiques et soins simulés». Six séances sont animées par une équipe de tuteurs paramédicaux portant sur : ponction veineuse, pose de voie veineuse périphérique, aspiration gastrique et endotrachéale par les étudiants en soins infirmiers (ESI) et radioprotection, analyse de radiographies standard et TDM par les élèves manipulateurs en électroradiologie médicale (EMERM). L'évaluation est basée sur un auto-questionnaire de satisfaction renseigné par les tuteurs paramédicaux et les tutorés EM (Quizz Yourself, échelle de Likert de 1 à 3). Les tuteurs EM (étudiants de second cycle, qui encadrent les autres séances de simulation), observateurs lors de ces séances, sont également interrogés. Quinze questions concernant les compétences réglementaires des deux métiers paramédicaux sont posées aux tutorés EM, avec des réponses sous forme de Vrai/Faux, avant et après la formation.
Résultats : Au total, 31 tuteurs paramédicaux ESI et EMERM, 40 étudiants tutorés (EM) et 13 tuteurs $\mathrm{EM}$ en quatrième ou cinquième année ont participé à l'enquête. Les résultats montrent que $89 \%$ des participants sont favorables à ce type d'ateliers $(85 \%$ très satisfaits des ateliers de soins infirmiers), $35 \%$ demandent d'y consacrer plus de temps pour couvrir plus de thèmes. Un total de $69,7 \%$ des tuteurs paramédicaux (ESI et EMERM) se sont sentis confiants (12,1\% légèrement dépassés, $9,7 \%$ légèrement mal à l'aise). Ils ont déclaré avoir développé les compétences suivantes : enseignement $(45,1 \%)$, aisance à l'oral (35,5\%), accompagnement et écoute (32,3\%). Par ailleurs, les tuteurs EM qui ont observé les ateliers pensent qu'ils sont adaptés : leurs avis sont très favorables à $91,7 \%$ pour les ESI et $50 \%$ pour les EMEM. Les tuteurs EM doutent de leurs capacités à animer eux-mêmes les ateliers pris en charge par ESI $(91,7 \%)$, ou les EMERM $(100 \%)$.

D'autre part, les EM pensent que la formation leur a permis d'améliorer leurs connaissances des métiers paramédicaux. Cette sensation s'est révélée objective pour les compétences des ESI $(83,5 \%$ de bonnes réponses après, $78,2 \%$ avant) mais le métier d'EMERM reste plus méconnu (nombre de bonnes réponses inchangé : $45,2 \%)$.

Conclusion: Les étudiants en santé sont demandeurs de ces moments de formation pluri-professionnelle dès la formation initiale dont ils retirent une réelle satisfaction en termes d'acquisitions de compétences. Il est probable que ces rencontres facilitent la synergie future et changent le regard porté sur la profession de l'autre avec un respect des compétences mutuelles. Des études complémentaires relatives aux représentations sociales pourraient confirmer cette hypothèse.

Mots-clés : évaluation, interprofessionalité, tutorat

$\mathrm{CO} 02$

\section{La collaboration interprofessionnelle dans l'enseignement de la prise en charge d'un patient poly-pathologique en soins premiers}

\section{Emile ESCOURROU, Bruno CHICOULAA, André STILLMUNKES, Marie-Eve ROUGE-BUGAT, Pierre MESTHE, Stéphane OUSTRIC}

Département universitaire de médecine générale, Université Paul-Sabatier - Toulouse 3, Faculté médecine Rangueil, Toulouse, France

Contexte : En 2013, le Collège national des généralistes enseignants publie 11 familles de situation correspondant aux «situations auxquelles les internes devront être confrontés durant leur formation afin d'acquérir les savoir-agir leur permettant de remplir les missions que la société attend d'eux». La prise en charge d'un patient atteint de poly-pathologies en soins premiers était une de ces situations. 
En complément de l'enseignement pratique de terrain, le département universitaire de médecine générale (DUMG) de Toulouse souhaitait construire un enseignement facultaire dédié à ce thème. Cet enseignement s'inscrit dans le cadre du parcours professionnalisant basé sur l'acquisition des ressources, des pratiques professionnelles et de la réflexivité.

Objectif : Construire un enseignement facultaire d'une journée sur la prise en charge d'un patient poly-pathologique en soins premiers.

Méthode: Un groupe de travail de 3 enseignants de médecine générale, un responsable pédagogique du DUMG, 2 représentants d'internes, deux infirmières et un masseur-kinésithérapeute a été constitué.

Le groupe de travail s'est réuni deux fois trois heures pour établir : les objectifs de l'enseignement, le déroulé pédagogique, les messages clefs, l'organisation et le déroulement des interventions de chaque enseignant.

Résultats : Le groupe de travail a proposé un enseignement basé sur une collaboration interprofessionnelle : médecin-infirmier-masseurkinésithérapeute. La collaboration entre ces trois professions était le reflet de la réalité de terrain pour la prise en charge d'un patient polypathologique à domicile.

L'objectif pédagogique principal de l'enseignement était de programmer une prise en charge globale, pluri-professionnelle, hiérarchisée, négociée, et inscrite dans le temps pour un patient polypathologique.

Les objectifs pédagogiques secondaires étaient : (1) établir un plan de santé en collaboration avec les professionnels de santé intervenant au domicile, (2) prévenir la iatrogénie chez un patient poly-pathologique, (3) connaître les objectifs des soins les plus fréquent de kinésithérapie, savoir communiquer avec les masseurs kinésithérapeute, et savoir les prescrire, (4) connaître les principaux pansements, leurs utilisations, savoir communiquer avec les infirmiers, prescrire les soins infirmiers.

L'enseignement était destiné aux internes en dernière année de DES avec quatre groupes répartis, sur 4 dates.

Une pédagogie active devait être favorisée en divisant chacun des 4 groupes en sous-groupes de 10 à 15 internes lors de la journée d'enseignement.

L'apprentissage par résolution de problèmes était basé sur 3 situations cliniques authentiques recouvrant 3 différents temps de prise en charge d'un même patient.

L'évaluation était réalisée par un recueil des attentes des internes en début d'enseignement puis une vérification des réponses aux attentes en fin de journée.

Discussion et conclusion : La collaboration interprofessionnelle pour cet enseignement a pu permettre un échange entre les acteurs de terrain d'aujourd'hui et les futurs médecins généralistes. En plus de l'acquisition de compétences spécifiques à la prise en charge d'un patient poly-pathologique, les internes pouvaient acquérir des compétences communicationnelles et relationnelles avec les professionnels paramédicaux intervenant au domicile de leurs futurs patients.

Il pourrait être pertinent de proposer l'intervention d'un patient expert pour compléter l'enseignement.
$\mathrm{CO} 03$

\section{Projet DÉCLIC (Développement de l'enseignement clinique et communautaire, Canada-Mali); une expérience de formation en interdisciplinarité en urgence, trauma, transferts sécuritaires des patients (UTT)}

\author{
Anne GAGNON ${ }^{1}$, Mamadou Bayo COULIBALY², \\ Aminata SANGARÉ ${ }^{3}$ \\ ${ }^{1}$ Formation continue, services aux entreprises et international, \\ Cégep de Saint-Jérôme, Saint-Jérôme, Canada \\ 2 Encadrement clinique, Centre de santé communautaire \\ universitaire de Konobougou, Bamako, Mali \\ ${ }^{3}$ Animation pédagogique, Institut national de formation en \\ sciences de la santé, Bamako, Mali
}

Contexte : Depuis 2011, le projet DÉCLIC, piloté par un consortium canadien en soins de santé (Centre de coopération internationale en santé et développement, Cégep de Saint-Jérôme et université de Sherbrooke), travaille à l'amélioration de l'offre de services de santé de première ligne, tant en milieu urbain qu'en milieu rural, principalement au bénéfice des femmes et des enfants.

Objectif : La collaboration entre les partenaires du projet DÉCLIC a pour objectif d'offrir une formation spécialisée en UTT, conçue et enseignée en multidisciplinarité, s'adressant au personnel des soins de santé de première ligne.

Méthode: Le projet de formation en UTT est né d'un besoin d'amélioration des capacités d'intervention en soins d'urgence du personnel de première ligne dans les centres de santé communautaires universitaires (CSCom-U). Pour ce faire, les intervenants au projet DÉCLIC ont convenu de produire un premier module de formation interdisciplinaire, avec des médecins, infirmiers et infirmières œuvrant au sein du projet. Les techniques de soins qui semblaient les plus importantes ont été identifiées. Par la suite, ces techniques ont été validées lors d'une rencontre réunissant divers acteurs du milieu de la santé au Mali, notamment des infirmières, des médecins et un représentant de la protection civile. La formation des formateurs fut également planifiée à ce moment.

La $2^{\mathrm{e}}$ phase de préparation a été menée au Canada par Sylvie Marsan, infirmière, en collaboration avec Dr François Couturier. Cette rencontre a rassemblé des spécialistes canadiens et maliens et avait pour objectif de :

- valider le contenu développé pour le module de formation;

- compléter les outils pédagogiques;

- compléter la formation des formateurs ;

- tenir un atelier de validation avec les formateurs identifiés.

Ce travail terminé, la formation s'est déployée en cascade sur le terrain. La cohorte formée au Canada a initié une équipe de formateurs au premier module UTT. Cette dernière était chargée d'enseigner l'UTT au personnel des 5 CSCom-U et aux enseignants de l'Institut national de formation en sciences de la santé (INFSS). Le travail s'est ensuite poursuivi par l'adaptation du module de 
formation UTT pour les différents intervenants en santé dans les villages, avec tous les défis que cela a comportés.

Résultats : Depuis janvier 2016, l'équipe UTT a permis la formation de près de 130 intervenants dans les différents CSCom-U et villages avoisinants.

L'INFSS et les CSCom-U ont développé une importante expertise au niveau de l'enseignement interdisciplinaire des premiers soins en UTT. Le travail se poursuit toujours sur le terrain et le développement d'un deuxième module de formation UTT est en cours.

Discussion et conclusion : Forte de son expérience d'élaboration de formation interdisciplinaire en UTT, les partenaires du projet canadien DÉCLIC, souhaitent la partager. La collaboration canadomalienne a donné lieu à des échanges d'expertises et de pratiques au bénéfice du personnel des CSCom-U et de l'INFSS ainsi qu'à celui de la population malienne. L'expérimentation de ce modèle de formation interdisciplinaire en cascade nous semble très prometteuse pour de futurs projets en santé de $1^{\text {re }}$ ligne.

Mots-clés : interdisciplinarité, soins d'urgence, formation de soins de santé, formation de formateurs, soins de santé $1^{\text {re }}$ ligne en Afrique

\section{$\mathrm{CO} 04$}

\section{Des occasions d'apprentissage interprofessionnel à valoriser dans les milieux de stage}

\section{Véronique LISÉE, Claudia TALBOT-COULOMBE, Diane CLAVET, Chantal LEMIRE, Caroline BOIS, Marie-Josée APRIL, Hélène CORRIVEAU}

Structure d'appui à la formation à la collaboration professionnelle, Vice-décanat au développement pédagogique et professionnel, Université de Sherbrooke, Sherbrooke, Canada

Contexte : Intégrer formellement l'enseignement-apprentissage à la collaboration professionnelle (CP) en prenant appui sur la participation d'étudiants issus de programmes différents représente un défi majeur pour les programmes de formation en santé. Actuellement, le parcours de développement de la compétence à la $\mathrm{CP}$ n'est pas explicite pour les étudiants et les programmes misent principalement sur les stages pour fournir des occasions d'apprentissage interprofessionnel. Toutefois, ces occasions d'apprendre à propos de l'autre, avec l'autre et de l'autre ne sont pas réellement formalisées ni pleinement optimisées. Il devient important de faire émerger le «curriculum caché» présent dans les parcours de professionnalisation, afin de valoriser les pratiques exemplaires déjà en place dans les milieux de stage.

Objectifs : Identifier et rendre explicite les occasions d'apprentissage interprofessionnel vécues lors des stages par les étudiants de $1^{\text {er }}$ cycle universitaire de différents programmes de formation en santé.

Méthode : Sondage électronique comportant 22 questions, dont des choix multiples et des questions à court et long développement, transmis par courriel (printemps et automne 2016) aux étudiants des programmes d'ergothérapie, de physiothérapie, de sciences infirmières et de médecine. Une analyse de contenu avec codage semi-ouvert et une analyse quantitative descriptive ont été réalisées pour identifier les milieux et les situations propices à l'apprentissage de la $\mathrm{CP}$, le niveau d'implication des étudiants dans les situations et les visées d'apprentissage reconnues par les étudiants lors de ces occasions.

Résultats : Le sondage a été complété par 535 participants $(57,34 \%)$ parmi les 933 étudiants approchés. Les étudiants ont principalement mentionné trois milieux de stage comme étant propices à y vivre des situations de CP qui gagneraient à être valorisées, soit: les milieux hospitaliers (unité de soins) $(30 \%)$, le soutien à domicile ( $18 \%)$ et les centres de réadaptation $(11 \%)$. Plus que simples observateurs, la majorité des étudiants (73\%) étaient directement impliqués dans les situations décrites de CP. Les opportunités d'apprentissage soulignées par les répondants sont les rencontres multidisciplinaires récurrentes dans le temps, la réalisation de plans d'intervention et les évaluations conjointes multidisciplinaires. Également, la santé mentale, la pédiatrie et la gériatrie ressortent comme des domaines de pratiques cliniques où la $\mathrm{CP}$ est déjà bien établie. Selon les répondants, les visées d'apprentissages ayant été particulièrement activées lors de ces situations de collaboration sont: «Travailler en collaboration» $(31 \%)$, «Communiquer avec d'autres» $(27 \%)$ et «Clarifier les rôles» (17\%). Lorsqu'on leur demande de décrire ce qu'ils y ont appris, les éléments liés à la clarification des rôles dominent dans l'analyse qualitative de leurs énoncés.

Discussion et conclusion : Cette banque de situations identifiées par les étudiants témoigne d'exemples concrets de manifestations de la $\mathrm{CP}$ dans les milieux de stage et pourra servir ultérieurement au développement de différentes activités de formation y compris de formation professorale. De plus, les situations pourront être formalisées pour faire partie d'une sélection d'activités intégrée à un parcours de développement de la compétence à la $\mathrm{CP}$ et mise à la disposition de l'étudiant pour développer son agir compétent en matière de $\mathrm{CP}$.

Mots-clés : collaboration interprofessionnelle, stages, formation en santé

\section{$\mathrm{CO} 05$ \\ Le modèle interprofessionnel de Lausanne}

\author{
David GACHOUD ${ }^{1}$, Leopoldo LUCARELLI ${ }^{2}$, Serge \\ GALLANT ${ }^{3}$, Nadine OBERHAUSER ${ }^{4}$, Anne-Claude ALLIN ${ }^{5}$ \\ ${ }^{1}$ Faculté de biologie et de médecine, Université de Lausanne, \\ Lausanne, Suisse \\ ${ }^{2}$ Département de médecine, Centre hospitalier universitaire \\ vaudois, Lausanne, Suisse \\ 3 Centre des formations, Centre hospitalier universitaire \\ vaudois, Lausanne, Suisse \\ ${ }^{4}$ Haute École de Santé Vaud, Lausanne, Suisse \\ ${ }^{5}$ Haute École de Santé La Source, Lausanne, Suisse
}

Contexte et problématique : La pratique collaborative ou collaboration interprofessionnelle dans les milieux de soins n'est pas toujours performante et a une incidence sur la qualité des soins et sur la sécurité des patients. Il y a aujourd'hui une corrélation démontrée 
entre la qualité de la collaboration dans les milieux cliniques et la qualité des soins aux patients (Manser, 2009; Mazocco et al., 2009; Gittel et al., 2000; Wiegmann et al., 2007; Saltvedt et al., 2002 ; Borill et West, 2011). Des études montrent que l'éducation interprofessionnelle, à savoir des séquences de formation dans lesquelles « des membres de deux ou plusieurs professions sont réunis pour apprendre avec les autres, grâce aux autres et à propos des autres » (Traduction libre, CAIPE, 2016), a un impact positif sur la collaboration interprofessionnelle (Morey et al., 2002 ; Reeves et al., 2016).

Description : Quatre institutions de formation et de soins vaudoises, la Haute École de Santé Vaud, la Haute École de la Santé La Source, la faculté de biologie et de médecine de l'université de Lausanne et le centre hospitalier universitaire vaudois, se sont regroupées pour fonder le Groupe interinstitutionnel d'Éducation et de Pratique Interprofessionnelles (GEPI). Ce groupe a travaillé au développement de projets-pilotes en éducation interprofessionnelle et, en parallèle, à l'élaboration d'un modèle interprofessionnel, le modèle de Lausanne, modèle reliant éducation et pratique interprofessionnelles.

Dans les milieux de l'éducation, toute une gamme de modèles ont été développés pour illustrer graphiquement une idée, un concept, voire même une théorie. Prenons par exemple la pyramide de Miller ou le cycle d'apprentissage de Kolb. Leurs auteurs recourent à une représentation graphique pour soutenir l'explicitation d'une idée, d'un concept ou d'une théorie qu'ils proposent. En matière d'interprofessionalité, les quelques modèles existants ne semblent pas toujours répondre aux besoins concrets des formateurs désireux de mettre en place une formation interprofessionnelle ou d'améliorer la pratique collaborative dans un milieu clinique. Partant de modèles existants, d'une revue intégrative de la littérature et de leurs expériences pédagogiques et professionnelles, les auteurs ont décrit un modèle innovant comprenant les éléments à considérer impérativement pour mettre sur pieds des séquences d'éducation interprofessionnelle et pour développer de réelles pratiques collaboratives. L'objectif final reste bien sûr celui d'atteindre la meilleure qualité de soins possible.

C'est ce modèle qui sera présenté et il sera illustré par quelques réalisations concrètes en matière d'éducation interprofessionnelle.

Mots-clés : éducation, collaboration, interprofessionalité

\section{CO 06}

\section{Le Continuum des pratiques interprofessionnelles en santé et services sociaux : un outil inestimable pour la formation interprofessionnelle}

\author{
Emmanuelle CAREAU ${ }^{1,2}$, Nathalie BRIÈRE ${ }^{3,4}$, Line \\ PARÉ $^{3,4}$, Élise MILOT ${ }^{1,3}$ \\ ${ }^{1}$ Université Laval, Québec, Canada \\ 2 Centre interdisciplinaire de recherche en réadaptation et \\ intégration sociale (CIRRIS), Québec, Canada
}

${ }^{3}$ Réseau de collaboration sur les pratiques interprofessionnelles (RCPI), Québec, Canada

${ }^{4}$ Centre intégré universitaire de santé et de services sociaux (CIUSSS) de la Capitale-Nationale, Québec, Canada

Contexte: Selon l'OMS, le développement d'une main-d'œuvre ayant développé ses compétences collaboratives est le déterminant central d'une collaboration interprofessionnelle (CIP) efficace. Cependant, pour être en mesure d'encadrer les pratiques interprofessionnelles, nous devons assurément nous appuyer sur une définition claire et précise du phénomène. Or, il n'existe pas nécessairement de conceptualisation consensuelle au sein de la communauté scientifique. Que ce soit dans les milieux de pratique, d'enseignement ou dans les écrits scientifiques, les termes utilisés sont souvent confondus ou utilisés sans distinction : interdisciplinarité, multidisciplinarité, travail d'équipe, coopération... De plus, s'il est assez facile d'identifier dans les écrits scientifiques les facteurs qui facilitent et contraignent la collaboration, il est beaucoup plus difficile de décrire précisément les pratiques devant être adoptées par les professionnels pour répondre de façon cohérente et intégrée aux besoins des patients.

Objectifs: Cette communication sera l'occasion de présenter le processus de développement du cadre de référence «Le Continuum des pratiques interprofessionnelles en santé et services sociaux » qui illustre comment les professionnels devraient collaborer pour obtenir des résultats optimaux de santé et de bien-être pour les populations. Nous exposerons aussi de quelle façon ce cadre illustrant les processus interactionnels de collaboration est utilisé comme outil pédagogique dans les cursus universitaires et les formations continues.

Méthode : Ce cadre de référence a été élaboré par la conjugaison des savoirs théoriques provenant des écrits scientifiques et des savoirs expérientiels de différents acteurs (chercheurs, enseignants, professionnels et patients). Un processus systématique en sept étapes a été utilisé pour son développement et sa validation. Son utilisation depuis maintenant près de six ans auprès d'étudiants de dix programmes des sciences de la santé et services sociaux; ainsi qu'auprès d'une centaine d'équipes interprofessionnelles, a permis de documenter de façon rigoureuse son apport à la formation interprofessionnelle.

Résultats: Ce cadre de référence illustre cinq types de pratiques différentes selon quatre axes distincts: la situation, l'intention, les interactions et les savoirs disciplinaires. La logique générale entre les quatre axes qui évoluent sur un continuum peut être expliquée ainsi : la situation de la personne, de ses proches ou de la communauté nécessite une collaboration plus ou moins étroite entre les professionnels selon son degré de complexité. Les professionnels identifient leur intention de collaboration (ex. échange d'information, concertation, prise de décision commune), ce qui détermine les interactions requises entre les individus, créant une interdépendance plus ou moins intense. Cette interaction et cette interdépendance impliquent une mise en commun des savoirs disciplinaires (approche uni-, multi- ou interdisciplinaire), qui permet de répondre de façon optimale aux besoins de la personne, de ses proches ou de la communauté. 
Discussion et conclusion: Ce cadre de référence est maintenant utilisé pour soutenir la formation interprofessionnelle destinée aux étudiants de toutes disciplines dans plusieurs universités du Québec (Canada), ainsi que la formation continue s'adressant aux professionnels en exercice. Plusieurs chercheurs, consultants et décideurs à travers le monde l'utilisent pour rédiger des documents d'orientation sur la collaboration, pour accompagner les équipes interprofessionnelles ou pour mener des recherches sur le sujet.

Mots-clés : collaboration interprofessionnelle, formation interprofessionnelle, continuum des pratiques interprofessionnelles, réponse cohérente et concertée aux besoins

\section{$\mathrm{CO} 07$}

\section{La pratique réflexive en collaboration interprofessionnelle : apprendre à réfléchir ensemble pour mieux collaborer}

\author{
Amélie RICHARD ${ }^{1,2}$, Emmanuelle CAREAU ${ }^{1,2}$, Mathieu \\ GAGNON $^{3,4}$ \\ ${ }^{1}$ Université Laval, Québec, Canada \\ ${ }^{2}$ Centre interdisciplinaire de recherche en réadaptation et \\ intégration sociale (CIRRIS), Québec, Canada \\ ${ }^{3}$ Université de Sherbrooke, Sherbrooke, Canada \\ ${ }^{4}$ Centre de recherche interuniversitaire sur la formation et la \\ profession enseignante (CRIFPE), Montréal, Canada
}

Contexte: Dans le domaine de la santé et des services sociaux, la collaboration interprofessionnelle (CIP) constitue une façon privilégiée de répondre aux besoins des patients de façon cohérente et adaptée. La concertation entre les partenaires n'est cependant pas toujours simple à actualiser et la nécessité d'adopter des actions concrètes pour soutenir les pratiques de CIP apparaît incontournable. À cet effet, un des moyens identifiés est le développement de la pratique réflexive chez les intervenants. La pratique réflexive est généralement définie comme étant un processus par lequel un professionnel, face à un doute ou à un problème, réfléchit sur son action et recherche des solutions possibles. Peu d'études ont toutefois été menées sur l'utilisation de la pratique réflexive en contexte de CIP, où la réflexion est menée par l'ensemble de l'équipe.

Objectifs: Une recension systématique a été conduite afin de documenter l'efficacité de la pratique réflexive en contexte de CIP (Objectif 1), et de décrire les modalités et les méthodes de pratique réflexive utilisées (Objectif 2).

Méthode : Les articles ont été retenus selon les critères d'inclusion suivants: articles scientifiques avec expérimentation et mesure des résultats dans le domaine de la santé et des services sociaux, contexte de collaboration interprofessionnelle et intégration explicite d'une méthode de pratique réflexive basée sur une théorie ou des critères précis. Concernant l'objectif 1, l'analyse de l'efficacité de la pratique réflexive en IPECP a été faite sur la base de la «Jet Classification of Outcomes » (Freeth et al.). Concernant l'objectif 2, les modalités et les méthodes de pratique réflexive utilisées en IPECP ont été identifiées de façon déductive et inductive.
Résultats : Sur un total de 2231 articles trouvés, six ont été retenus. Concernant l'objectif 1 , des résultats positifs ont été retrouvés dans l'ensemble des niveaux de la Jet Classification of Outcomes (appréciation de l'activité, développement d'habiletés, changements de comportements, amélioration des trajectoires de soins...). Concernant 1'objectif 2, les principales caractéristiques des interventions de pratique réflexives retrouvées dans les différents articles ont été identifiées : une intervention fondée sur une théorie réflexive ou un processus réflexif rigoureux et explicite, le recours à la théorie ou la théorisation pour améliorer les pratiques (theory to practice), l'utilisation de situations cliniques ou de récits comme amorce au processus, la présence d'un facilitateur lors des discussions en groupe de même qu'une rétroaction offerte sur le fonctionnement des équipes et des discussions réflexives.

Discussion et conclusion: Le nombre important de recherches éliminées suite à l'application des critères d'inclusion semble indiquer que la question de la pratique réflexive paraît de plus en plus présente en CIP, mais que son application ou l'évaluation de ses impacts demeurent peu documentés explicitement. La pratique réflexive semble avoir des effets intéressants en CIP, mais les preuves en ce sens sont encore limitées dÛ entre autres à de petits échantillons et à la validité des instruments utilisés. Des recherches supplémentaires sont nécessaires pour documenter l'efficacité et les conditions optimales de la pratique réflexive en CIP.

Mots-clés : pratique réflexive, collaboration interprofessionnelle, formation interprofessionnelle

\section{2. Éducation thérapeutique}

\section{$\mathrm{CO} 08$ \\ Création d'un programme d'éducation des malades obèse sur le parcours préparatoire avant une chirurgie bariatrique}

\author{
Geraud TUYERAS ${ }^{1}$, Mael CHALRET DU RIEU ${ }^{2}$, Nicolas \\ CARRERE $^{2}$, Bertrand SUC ${ }^{1}$, Fabrice MUSCARI ${ }^{1}$ \\ ${ }^{1}$ Chirurgie viscérale, CHU Rangueil, Toulouse, France \\ ${ }^{2}$ Chirurgie viscérale, CHU Purpan, Toulouse, France
}

Contexte: L'obésité représente actuellement un problème de santé publique en constante aggravation. Près de $25 \%$ de la population est en situation d'obésité. La chirurgie représente la manière la plus efficace et durable de perdre du poids avec comme objectifs, une amélioration de la qualité de vie, des comorbidités associées et de l'espérance de vie. Cependant, elle n'intervient qu'au terme d'une préparation longue d'une durée minimale de 6 mois. La première consultation chirurgicale est très importante, son but est de réaliser le dossier médical, le bilan clinique et nutritionnel, d'informer les patients sur les détails du parcours, des interventions, de leur risque et de leurs contraintes.

Objectif : L'objectif de cette étude était d'apporter une information détaillée sur le parcours ainsi que sur les interventions avant la $1^{\text {re }}$ consultation, afin de se focaliser, le jour de la consultation, sur 
l'examen clinique, et la clarification des informations non ou mal comprise.

Méthode : Un référentiel d'éducation a été créé pour les patients. Il leur était envoyé avant la $1^{\text {re }}$ consultation par e-mail. Ce référentiel était composé de 11 vidéos sonorisées et de 1 document écrit. L'e-mail était envoyé aux patients lorsqu'ils prenaient rendez-vous. Un questionnaire d'évaluation des connaissances portant sur les vidéos notées sur 9 points, et un de satisfaction étaient remplis dans la salle d'attente avant la consultation. Nous avons inclus la totalité des patients, satisfaisant aux critères d'une intervention chirurgicale, d'un chirurgien du service de chirurgie viscérale sur la période de janvier 2017 à juin 2018.

Résultat: Sur la période d'analyse, 38 patients (29\% d'homme), pour un âge médian de 40 ans (20 à 65 ans), l'IMC moyen était de $40 \mathrm{~kg} / \mathrm{m}^{2}$. Sur les 38 patients inclus, $23(60 \%)$ se sont présentés à la consultation chirurgicale et ont donc était analysé. Le score médian de connaissance était de 6/9. Une seule patiente n'a pas reçu le mail de formation Internet. Les scores médians de satisfactions (intérêt, clarté, efficience) sont évalués entre 9 et 10 sur un total de 10 , avec une préférence pour la partie vidéo (55\%). En moyenne les patients ont passé environ 45 minutes sur les vidéos. Sur le site Internet, les vidéos ont été vues en moyennes 57 fois (41 à 100 fois en fonction des vidéos). La consultation qui a suivie a pu être personnalisée sur les questions de chaque patient et jugée beaucoup moins répétitive par le praticien sans perte d'information.

Discussion et conclusion : L'éducation des malades obèses concernant le parcours préopératoire avant chirurgie grâce au TICE avant la première consultation semble une information efficace et appréciée par les patients avec des scores de compréhension satisfaisants et des indices de satisfactions élevés. Le nombre de connections sur les différentes vidéos montrent que les patients peuvent lire plusieurs fois les vidéos au cours de leur préparation. Cette étude se poursuit afin de confirmer, sur un effectif plus important, ces résultats.

Mots-clés : éducation thérapeutique, chirurgie bariatrique, obésité

\section{Dispositif de formation}

\section{CO 09}

\section{Le projet DESHMA : penser l'innovation pédagogique dans une perspective inclusive}

\author{
Agnès D'ARRIPE ${ }^{1}$, Cédric ROUTIER ${ }^{1}$, Ingrid FOURNY ${ }^{2}$, \\ Grégory AIGUIER ${ }^{3}$ \\ ${ }^{1}$ Unité HADEPAS, Institut catholique de Lille, Lille, France \\ 2 Ateliers d'Humanicité, Institut catholique de Lille, Lille, \\ France \\ ${ }^{3}$ Centre d'éthique médicale, EA 7446 Ethics, Faculté de médecine \\ et de maïeutique, Université catholique de Lille, Lille, France
}

Contexte et problématique: Dans cette communication, nous présenterons un projet innovant de formation inclusive consacrée à la déficience intellectuelle. Développé dans le cadre d'une campagne de soutien à des projets d'innovation pédagogique pilotée par l'institut catholique de Lille, le projet DESHMA (Développer la Sensibilisation au Handicap Mental par les Auto-représentants) poursuit deux objectifs. Il s'agit tout d'abord de construire un programme de formation original visant à sensibiliser les futurs professionnels du secteur sanitaire et médico-social à l'expérience de vie des personnes atteintes d'une déficience intellectuelle (leur vécu, leurs capacités, leurs compétences); concomitamment, ce projet vise à créer les conditions d'une participation effective des auto-représentants à la dynamique pédagogique.

La démarche engagée est animée d'une conviction forte. Les personnes atteintes d'une déficience intellectuelle participant au projet ne peuvent être uniquement mobilisées pour témoigner de l'expérience d'une vie avec leur déficience. Elles doivent être considérées comme des parties prenantes à la formation. Par conséquent, le développement de compétences pédagogiques de la part de ces personnes déficientes constitue un enjeu essentiel de ce projet. Sur le plan des fondements pédagogiques, ce dernier s'inscrit en cela dans une logique d'empowerment et d'autodétermination des personnes atteintes d'une déficience intellectuelle participant à la démarche. Celle-ci s'inscrit dans les orientations de la Convention de l'ONU relative aux Droits des Personnes Handicapées (CDPH) et dans la perspective d'une société appelée à devenir plus inclusive. Description : L'objectif de cette communication est pluriel. À partir d'un bref rappel des présupposés pédagogiques et des finalités de formation qui en découlent, il s'agira en tout premier lieu d'expliciter la démarche d'ingénierie pédagogique mise en place pour construire ce programme de formation. Nous présenterons notamment les séances d'intelligence collective, réunissant des personnes atteintes d'une déficience intellectuelle, des enseignants-chercheurs, des professionnels du secteur du handicap et des formateurs : ces séances ont permis la construction des objectifs spécifiques de la formation, l'identification des thèmes de cours ainsi que la constitution de binômes (une personne déficiente intellectuelle, un enseignant/ formateur). Nous présenterons le travail des binômes qui construisent leur cours à leur rythme, accompagnés à distance par l'équipe-projet. Des temps de regroupements en session plénière sont également organisés pour assurer une progression collective.

Évaluation: Nous présenterons enfin les premiers résultats de la démarche globale d'évaluation mise en œuvre, qui répond elle aussi à plusieurs objectifs. Une démarche d'évaluation certificative, tout d'abord, qui s'effectue à partir d'une simulation pédagogique et qui vise à évaluer les modalités pédagogiques (contenus, méthodes) choisies par les personnes constituant les binômes, mais aussi leur aptitude à dispenser le cours. Une évaluation autoformative, ensuite, construite à partir d'entretiens individuels qui visent à vérifier l'implication effective des deux personnes du binôme dans la construction du cours. Enfin, une évaluation contributive d'une gouvernance réflexive du projet, permettant à l'équipe-projet de comprendre la dynamique singulière à mettre en place en matière d'ingénierie pédagogique, compte tenu de la spécificité des parties prenantes à la démarche et qui doit permettre à l'équipe-projet de tirer 
les enseignements lui permettant de pérenniser le projet et de l'élargir à l'ensemble des filières de formation.

Mots-clés : empowerment, innovation pédagogique, handicap mental, autodétermination, co-élaboration

CO 10

\section{Proposition d'un cadre théorique pour la formation des médecins généralistes à l'incertitude, et des grilles d'évaluation qui en découlent}

\author{
Baptiste MOTTE ${ }^{1}$, Dominique VAN PEE ${ }^{2}$, Jean-Philippe \\ COBBAUT ${ }^{3}$ \\ 1 Département de médecine générale, Faculté libre de \\ médecine et maïeutique de Lille, Université catholique de \\ Lille, Lille, France \\ 2 Faculté de médecine, Université catholique de Louvain, \\ Bruxelles, Belgique \\ ${ }^{3}$ CEM - ETHICS EA 7446, Université catholique de Lille, Lille, \\ France
}

Contexte et objectifs: Chaque praticien peut être confronté à l'incertitude médicale. Du fait de la précocité de consultation en soins primaires, et de la confrontation à des symptômes isolés et peu spécifiques, pour le médecin généraliste l'incertitude tient une place importante dans le raisonnement clinique et la prise en charge. L'intolérance et/ou la mauvaise gestion de l'incertitude a des conséquences multiples: sur-prescription d'examens complémentaires ou de thérapeutiques à faible niveau de preuve, frein à la décision médicale partagée, insatisfaction professionnelle et risque de burn-out... Les internes en médecine générale devraient recevoir une formation à la gestion de l'incertitude qui permette de faire face à celle-ci. L'impact de cette formation devrait être évalué à l'aide de grilles adossées à un cadre théorique bien défini. Sur quel cadre théorique baser une formation des internes de médecine générale à l'incertitude? Quelles échelles choisir pour évaluer cette formation? Méthodes : Notre cadre théorique a été construit sur base des éléments synthétisant les informations recueillies au cours d'une recherche bibliographique sur l'incertitude, l'ambiguïté (terme recouvrant parfois le même concept dans la littérature), leurs origines, leurs conséquences, leurs (in)tolérances, les recommandations pour les gérer et les éventuels enseignements testés pour y faire face. Les grilles d'évaluation ont été sélectionnées et comparées à partir d'une recherche bibliographique par mots clés dans PUBMED (pour ne sélectionner que les travaux en lien avec le champ médical). Nous avons sélectionné et analysé les articles traitant de l'incertitude dans le contexte de la relation médecin-patient dans lesquels les chercheurs utilisent une échelle d'évaluation de l'incertitude ou de l'ambiguïté.

Résultats : Dans le cadre théorique retenu, l'incertitude est l'état dans lequel le praticien se trouve face à une situation ambiguë. L'intolérance à l'incertitude peut amener le praticien à des conduites d'évitement ou de déni de cette dernière dont la littérature a documenté l'impact négatif sur la prise en charge des patients et le bien-être des médecins. Enrichir l'épistémologie des étudiants à travers une formation contextualisée et s'assurer qu'ils aient les compétences de communication nécessaires pour partager cette incertitude avec le patient ou des confrères nous semblent être les principaux enjeux de cette formation. Concernant le choix de grilles d'évaluation, 530 articles émergent de la base de données avec les mots clés utilisés ; 90 sont sélectionnés d'après nos critères. Dix-neuf échelles sont mises en évidence: 7 investiguant l'ambiguïté et 12 investiguant l'incertitude. Les 2 grilles validées qui investiguent le mieux les enjeux principaux de notre modèle sont la «Physicians' Reaction to Uncertainty Scale» de Gerrity (ressenti vis-à-vis de l'incertitude et capacité à la communiquer) et la «Tolerance of Ambiguity in Medical Students And Doctors» de Hancock et al (évolution de la tolérance à l'ambiguïté en fonction de l'épistémologie).

Discussion et conclusion : Le cadre théorique élaboré, il nous reste à concevoir la formation. La pédagogie par concordance semble très intéressante pour enrichir l'épistémologie des étudiants en les exposant à différents contextes d'incertitude issus de la pratique quotidienne du médecin généraliste.

Mots-clés : incertitude, ambiguïté, évaluation, formation, médecine générale

\section{CO 11}

\section{Jouer pour mieux former les professionnels de santé: Est ce bien sérieux? Retours d'expériences de la Haute École de Santé La Source (Lausanne)}

\section{Dominique TRUCHOT-CARDOT}

Décanat innovation, Haute École de Santé La Source, Lausanne, Suisse

Contexte et problématique : De tout temps l'homme a joué. Le jeu est une des plus ancestrales sources d'apprentissage, de socialisation et de régulation «amiable» de conflits.

C'est au $X V^{\mathrm{e}}$ siècle, que le jeu sérieux prend son envergure pédagogique avec l'oxymore «Serio Ludere» qui renvoie à l'idée de traiter d'un sujet jugé sérieux avec une approche divertissante.

$\mathrm{Au}$ début de $\mathrm{XIX}^{\mathrm{e}}$ siècle, les armées britanniques et prussiennes choisissent de développer de nouvelles tactiques et former leurs officiers par le biais de la simulation ludique. Il en sera de même pour presque toutes les armées du monde jusqu'à l'avènement de l'informatique.

Le concept moderne de jeu sérieux informatisé remonte à 1947 avec la création du premier objet vidéo-ludique et n'a eu de cesse de se développer en intégrant progressivement toutes les fonctionnalités pédagogiques actuelles : diffusion d'un message, dispensation d'un entraînement et échange de connaissances entre joueurs.

Le serious game selon Julian Alvarez est « un dispositif, numérique ou non, visant un marché s'écartant du seul divertissement, proposant 
simultanément des mécaniques de jeu et fonctions utilitaires parmi les trois suivantes : diffusion de message(s), dispense d'entraînement(s) ou collecte de données ».

À ne pas confondre avec les serious diverting ou modding qui sont des jeux classiques, ludiques, détournés de leur usage premier à des fins sérieuses.

De fait, la gamification englobe donc l'action d'utiliser des jeux existants et de les exploiter à des fins pédagogiques.

La place du jeu est un enjeu majeur en santé.

En effet, l'enseignement des soins, tente d'abandonner ses modèles historiques, transmissifs ou comportementalistes, au profit d'une approche socio-constructiviste.

Cette approche impose de fait une interdépendance de l'apprentissage et de son contexte.

Or nous rencontrons de plus en plus de difficultés de mise en œuvre pratique de ce type d'apprentissage en contexte réel, de part:

- Le nombre croissant d'étudiants à nombre constant de places de stage, de patients et d'enseignants ;

- L'autonomisation des patients qui ne veulent plus être des «expérimentateurs» et que leur soient garanti sécurité et excellence en tout lieu et en tout temps;

- La maîtrise des coûts, imposant des formations les plus pointues possible, au plus grand nombre, dans le temps le plus court.

À cette réalité vient s'agréger le fait que nos étudiants sont des digitales natives qui veulent plus que tout un encadrement personnalisé et de proximité, alliant tutorat et réflexions entre pairs. L'écosystème d'enseignement des soins ne doit-il pas être réinventé ? Description : Fort de ce constat et en partant du préambule que la gamification participe à la construction du lien entre la théorie et la pratique, nous avons effectué depuis 2014 une analyse de nos pratiques enseignantes du calcul professionnel, de la sécurité des soins et du travail en équipe.

Cette communication sera l'occasion de vous présenter nos réflexions et nos approches innovantes, technologiques pour certaines, gamifiantes pour d'autres mais délibérément stimulantes pour toutes. Mais également de s'intéresser à l'évaluation du jeu comme outil de médiation pour enseigner en santé.

Mots-clés : gamification, serious game, formation infirmière, calcul professionnel, sécurité des soins, interprofessionalité

\section{CO 12}

\section{Exposition des internes en stage en} médecine générale de niveau 2 (SASPAS) à L'UPEC aux onze grandes familles de situations prévalentes en médecine générale

\section{Laurence COMPAGNON, Elodie CROCCI, Adrian CHABOCHE, Julien LE BRETON, Emilie FERRAT, Vincent RENARD}

Département de médecine générale, Université Paris Est Créteil, Créteil, France
Contexte et objectifs: Notre département de médecine générale (DMG) a fait le choix du modèle d'apprentissage par compétences. Il utilise le référentiel des 11 familles de situations publié en 2013par le Collège national des généraliste enseignants (CNGE) et auxquelles les internes devront être confrontés afin d'acquérir les savoirs agir nécessaires.

Notre hypothèse principale était une sous exposition des internes aux situations cliniques relevant d'une prise en charge de patients chroniques par rapport aux situations aiguës.

Méthode: Notre étude observationnelle a recueilli l'ensemble des actes réalisés par les quinze internes au cours de leur SASPAS à l'université Paris Est Créteil (UPEC) entre novembre 2014 et avril 2015, via l'analyse de leurs journaux de bord où étaient décrits brièvement tous les actes réalisés en autonomie. La cohérence du codage des actes, en familles de situation a été vérifiée sur un échantillon et triangulés en cas de difficultés.

Résultats : Le total d'acte recueilli était de 11251, soit en moyenne de 750 actes par étudiant. Au total, 14295 situations ont été dénombrées. Plus de la moitié des situations cliniques $(56,66 \%)$ rencontrées par les internes était en lien avec des problèmes aigus (programmés ou non, fréquents ou exemplaires), alors que les situations concernant des patients souffrant de pathologies chroniques ou polymorbidité à forte prévalence ne concernaient que $20,64 \%$ des situations rencontrées. La troisième famille de situation la plus rencontrée était en lien avec les aspect légaux, réglementaires ou déontologiques $(7,2 \%)$. Venaient ensuite les situations de génitalité et reproduction $(4,73 \%)$ et de suivi de l'enfant $(4,32 \%)$. Les autres familles de situation étaient très peu représentées (moins de $1 \%$ chacune).

Certaines situations n'ont pas pu être codées dans les 11 familles $(n=270 ; 1,89 \%)$, le plus souvent en rapport avec des situation de prévention chez l'adulte.

Il n'y avait pas de différence significative en corrélation avec le lieu de soin. Lorsque le maître de stage était une femme, les internes rencontraient significativement plus de situations en lien avec le suivi d'enfant, d'IVG, ou de souffrance au travail.

Discussion : La principale force de cette étude réside dans le recueil exhaustif des actes réalisés par les internes en SASPAS sur un semestre (taux de réponse de $100 \%$ ) et le codage systématique de tous ces actes en familles de situation. Le nombre élevé de situations donne de la validité à cette analyse. Des approximations dans les comptes-rendus rédigés par les internes ont pu induire un biais dans le codage. L'activité des médecins généralistes en termes de familles de situations n'a pas été étudiée dans la littérature. Toutefois, différents travaux s'intéressant à l'analyse de leur activité par d'autres outils retrouvent une proportion plus importante de motifs et résultats de consultations en lien avec des pathologies chroniques ou avec la prévention qu'en lien avec des problèmes aigus.

Conclusion: Notre hypothèse de sous exposition des internes SASPAS aux situations de patients atteints de pathologie chronique a été confirmée. Cela doit conduire à augmenter dans nos terrains de stage les files actives de patients chroniques pour les internes en SASPAS.

Mots-clés : famille de situation, compétences, internes, médecine générale 
CO 13

\section{Quels sont les freins et les motivations des jeunes médecins généralistes à devenir maître de stage des universités?}

\author{
Yann BRABANT, Pascal PARTHENAY, José GOMES, \\ Pascal AUDIER, Pierrick ARCHAMBAULT
}

Département de médecine générale, Faculté de médecine de Poitiers, Poitiers, France

Contexte: Les effectifs des maîtres de stage des universités (MSU) restent encore éloignés des besoins indispensables pour encadrer les internes de médecine générale et leur proposer à tous le stage en autonomie supervisée (SASPAS). La problématique est la même pour le $2^{\mathrm{e}}$ cycle avec un accès partiel au stage ambulatoire pour les externes, pourtant obligatoire. Il est donc encore nécessaire de motiver des médecins généralistes à devenir MSU. Les jeunes généralistes pourraient être une cible importante du recrutement car ils semblent exprimer leur volonté d'être MSU après leur installation dans des études récentes. Les motivations et freins des médecins à devenir MSU ont déjà été étudiés mais sans s'intéresser spécifiquement aux jeunes généralistes.

Objectif : L'objectif principal de notre étude était d'explorer et d'identifier les freins et les motivations qui déterminent l'implication des jeunes médecins généralistes de moins de 40 ans dans la maîtrise de stage.

Méthode: Étude qualitative par entretiens semi-dirigés réalisée auprès des médecins généralistes installés et âgés de moins de 40 ans. Résultats attendus : Certains freins pourraient être les mêmes que ceux retrouvés chez l'ensemble des médecins généralistes comme le manque de temps, la présence d'un tiers dans la relation médecinpatient ou la crainte du regard critique de l'interne. De même, certaines motivations communes pourraient être retrouvées comme le partage des connaissances, la stimulation intellectuelle, ou la revalorisation de la médecine générale. Des freins spécifiques aux jeunes médecins pourraient se dégager comme la difficulté à confier sa patientèle, et des motivations comme la satisfaction de leur propre expérience du stage ambulatoire ou le sentiment de «redevance pédagogique» vis-à-vis de la faculté qui les a formés. De nouvelles idées sont attendues comme dans toute méthode exploratoire.

Discussion et conclusion : L'optimisation des freins spécifiques et la valorisation des motivations des jeunes généralistes à devenir MSU pourraient permettre d'améliorer le recrutement dans cette population.

Mots-clés : corps enseignant, stage pratique guidé, internat et résidence, enseignement spécialisé en médecine, renouvellement du personnel

\section{$\mathrm{CO} 14$}

\section{«Renouveau pédagogique» en kinésiologie : implantation d'un programme orienté sur le développement des compétences des étudiants}

\author{
Adrien CANTAT ${ }^{1}$, Geneviève CHARLAND ${ }^{1}$, Francis \\ LALIME $^{2}$, Philippe CORBEIL ${ }^{2}$, Daniel TURPIN ${ }^{1}$
}

${ }^{1}$ Vice-décanat à la pédagogie et au développement professionnel continu, Université Laval, Québec, Canada

2 Département de kinésiologie, Université Laval, Québec, Canada

Contexte et problématique: Le programme de kinésiologie de l'université Laval a entamé son «Renouveau pédagogique » en 2013 à la suite de l'évaluation périodique du programme. Celui-ci a décidé de repenser complètement le design pédagogique du cursus en mettant l'accent sur le développement de compétences inspirées des rôles CanMEDS utilisés en médecine (Le Collège royal des médecins et chirurgiens du Canada, 2015). De fait, un programme articulé autour de six compétences à développer est en cours d'implantation depuis la session d'automne 2016. Le développement d'un programme par compétences nécessite une refonte des méthodes d'enseignement et d'évaluation (Scallon, 2004) et exige une réflexion profonde sur le profil souhaité de l'étudiant diplômé.

Cette communication aura pour objectif de présenter la démarche de développement du programme par compétences et d'évoquer ses impacts sur le design pédagogique.

Aucun ordre professionnel n'existant au Québec en kinésiologie, la première étape fut l'élaboration d'un référentiel de compétences visant à identifier les compétences nécessaires à acquérir par l'étudiant pour exercer la profession de kinésiologue à l'issue du cursus universitaire (Paquay, 1994). Ce référentiel a initié l'instauration d'une approche programme permettant la consolidation des liens logiques entre les cours tout en favorisant la cohérence du programme par l'intégration progressive des apprentissages chez les étudiants (Sylvestre et Berthiaume, 2013).

Description : Des modifications dans l'architecture pédagogique du programme ont donc été entreprises afin de privilégier le développement et l'évaluation des compétences des étudiants. De nouveaux cours longitudinaux intitulés «cours intégrateurs» (cinq cours) et « démarche clinique ( (trois cours), répartis sur l'ensemble du cursus, seront insérés à chacune des trois années du programme. Leur rôle est primordial puisqu'ils visent le développement des compétences à travers la mobilisation, par les étudiants, de différentes ressources (Roegiers, 2000; Tardif, 2006) et la mise en application concrète des notions théoriques abordées dans les cours «traditionnels » davantage axés sur l'acquisition de connaissances. En outre, ces deux types de cours longitudinaux ont en commun l'exposition des étudiants à des situations d'apprentissage reflétant la réalité clinique de leur future profession.

Des méthodes d'évaluation différentes, comme des ECOS (Harden et Gleeson, 1979), des vignettes cliniques, des études de cas, permettant de porter un jugement sur les aspects cliniques ainsi que sur des compétences plus génériques (communication, professionnalisme), sont aussi en cours d'élaboration.

Par ailleurs, celles-ci permettront de documenter formellement le développement des compétences des étudiants en alimentant une plateforme numérique de suivi intitulée «Suivi du développement des compétences ». Durant les trois années d'études, chaque étudiant recevra régulièrement un tableau de suivi lui fournissant un portrait global du développement de ses compétences. En outre, dès le début du cursus, chaque étudiant sera associé à un tuteur avec qui il 
effectuera un suivi formel lors de rencontres planifiées. L'étudiant devra préalablement réaliser son auto-évaluation avant d'en discuter avec son tuteur et de déterminer si nécessaire, sur la base du tableau de suivi, des mesures d'amélioration (exercices à réaliser, exposition clinique à augmenter, etc.). Le programme de kinésiologie intègre ainsi dans son cursus un processus d'encadrement personnalisé des étudiants.

Mots-clés : approche programme, design pédagogique, compétence

CO 15

\section{Mise en place d'un nouveau programme de baccalauréat en sciences biopharmaceutiques : défis et réalisations 7 ans après le lancement}

Brian WHITE-GUAY, Sylvie PERREAULT, Marc SERVANT, Denis DE BLOIS

Faculté de pharmacie, Université de Montréal, Montréal, Canada

Contexte et problématique: La formation dans la $R \& D$ du médicament varie d'un pays à l'autre et se concentre le plus souvent sur une formation disciplinaire spécialisée au $2^{\mathrm{e}}$ ou $3^{\mathrm{e}}$ cycle universitaire. La mise en place d'un programme de formation de trois ans offert au $1^{\text {er }}$ cycle universitaire en sciences biopharmaceutiques à la faculté de pharmacie de l'université de Montréal constitue une approche inédite visant à offrir des connaissances de base multidisciplinaires sur le médicament permettant de mieux comprendre les enjeux importants allant de l'identification de la cible au remboursement du produit. Ce programme a été conçu pour répondre à un besoin de main d'œuvre qualifiée suite à une consultation élargie avec les milieux industriels du secteur biopharmaceutique au Québec alors que la faculté de pharmacie connaissait un profond changement avec l'introduction d'une nouvelle formation de premier cycle en pharmacie (Pharm. D) davantage orientée sur les soins et services pharmaceutiques.

Description: Depuis son lancement en 2009, le programme de Baccalauréat en sciences biopharmaceutiques offre une formation fondamentale et appliquée ayant comme fil conducteur le processus de découverte et développement du médicament. En revanche, cette formation ne permet pas d'accéder à la pratique professionnelle de la pharmacie.

Sur le plan pédagogique, ce programme comporte plusieurs types d'activités d'apprentissage: cours disciplinaires de type magistral, des travaux pratiques en chimie analytique, pharmacologie, pharmacocinétique et en biologie moléculaire, des cours d'intégration sur le développement préclinique et clinique (Phase I-IV) à l'aide d'études de cas réels puisées directement sur les sites publics d'agences internationales de réglementation du médicament. Il comporte également des projets de recherche en équipe, des présentations orales et finalement des ateliers pratiques visant à mieux connaître les opportunités de carrière dans le domaine biopharmaceutique.
Durant la troisième année, les étudiants peuvent opter pour un cheminement «Honor» conduisant aux études graduées, ou bien effectuer un stage de 4 mois dans des milieux industriels, gouvernementaux ou hospitalo-universitaires et soumettre un rapport détaillé selon les exigences généralement applicables pour une publication. Une importance particulière est accordée tout au long du programme au développement des compétences transversales avec des évaluations régulières comportant une rétroaction d'équipe et/ou individualisée. Par ailleurs, une collaboration originale de formation appliquée avec la bibliothèque de la santé de l'université de Montréal permet des interventions répétées visant la formation en recherche documentaire et en pratique factuelle reliée à des objectifs de cours offerts tout au long de la formation. Le programme reçoit annuellement près de 500 candidatures provenant majoritairement du niveau collégial pour environ 75-80 admissions. Parmi les étudiants admis, environ $50 \%$ atteignent la graduation, $35 \%$ transfèrent dans d'autres programmes (pharmacie, médecine, etc.) et $15 \%$ abandonnent. Après la graduation, plus de $70 \%$ poursuivent aux études supérieures, $25 \%$ s'orientent vers d'autres programmes professionnels et une minorité seulement entrent directement sur le marché du travail.

L'expérience acquise depuis le lancement du programme et les modifications apportées pour améliorer sa pertinence nous permettent de conclure qu'il s'agit d'une offre de formation originale, pertinente et susceptible de contribuer favorablement aux besoins de la relève dans le secteur biopharmaceutique.

Mots-clés : biopharmaceutique, médicaments, recherche et développement, formation

\section{CO 16}

\section{L'enseignement de la médecine narrative : un retour vers le futur?}

\section{François GOUPY ${ }^{1,2}$, Gaëlle ABGRALL ${ }^{3}$, Anne CHAHWAKILIAN $^{4}$, Elisabeth ASLANGUL ${ }^{5,6}$, Serge PERROT $^{7,8}$, Claire LE JEUNNE ${ }^{9,10}$}

${ }^{1}$ Service de santé publique, Hôtel Dieu de Paris, Assistance publique-Hôpitaux de Paris, Paris, France

2 Santé publique, Faculté de médecine Paris Descartes, Paris, France

${ }^{3}$ Service de psychiatrie, hôpital Tenon, Assistance publiqueHôpitaux de Paris, Paris, France

${ }^{4}$ Service de gérontologie, hôpital Broca, Assistance publiqueHôpitaux de Paris, Paris, France

${ }^{5}$ Service de médecine interne, hôpital Louis-Mourier, Assistance publique-Hôpitaux de Paris, Paris, France

${ }^{6}$ Médecine interne, Paris, France

${ }^{7}$ Service d'évaluation de la douleur, hôpital Tenon, Assistance publique-Hôpitaux de Paris, Paris, France

${ }^{8}$ Rhumatologie, Faculté de médecine Paris Descartes, Paris, France

9 Service de médecine interne, Hôpital Cochin, Assistance publique-Hôpitaux de Paris, Paris, France 
10 Médecine interne, Faculté de médecine Paris Descartes, Paris, France

Contexte et problématique : Dans le cadre de l'enseignement de médecine narrative proposé aux étudiants inscrits en quatrième année de médecine à la faculté de médecine Paris Descartes, une étude d'intervention par tirage au sort visant à comparer différentes modalités pédagogiques (écriture réflexive versus lecture critique d'articles, et lecture de trois textes écrits par des patients versus lecture de trois textes d'écrivains) a été organisée en 2012-2013.

Description: Partant des résultats de cette expérience, tous les étudiants de quatrième année participent désormais à des ateliers d'écriture réflexive et doivent lire deux textes choisis librement parmi les trois textes écrits par des patients et trois textes d'écrivains qui avaient été retenus dans l'étude initiale. La présentation de cette expérience sera faite en trois temps. D'abord la maquette de cet enseignement optionnel proposé pour la première fois en 2009-2010 à cinquante étudiants sera décrite et située par rapport aux enseignements de sciences humaines et sociales existants dans le cursus. Ensuite les enseignements de cette expérimentation qui a duré trois ans avant d'être proposée à tous les étudiants de quatrième année seront discutés en termes d'acceptabilité par les étudiants et de formation des chargés d'enseignements dirigés. Enfin les résultats seront donnés sous la forme d'une analyse des scores de satisfaction et des commentaires des étudiants vis-à-vis des ateliers d'écriture et des trois types de lecture testés pendant l'année 2012-2013, et d'une analyse des préférences de lecture des étudiants quand ils furent libres de choisir leurs lectures les années suivantes.

Ces résultats seront analysés en fonction du sexe et du projet de carrière de l'étudiant, et discutés par rapport aux objectifs pédagogiques d'apprentissage de l'écoute des patients et de réflexion sur le métier de médecin.

Mots-clés : relation médecin-malade, empathie, médecine narrative

CO 17

\section{Master sciences cliniques infirmières: retour d'expérience et perspectives}

\author{
Sébastien COLSON ${ }^{1,2}$ \\ 1 DUSI, Faculté de médecine, Aix-Marseille Université, \\ Marseille, France \\ 2 Faculté de sciences infirmières, Université de Montréal, \\ Montréal, Canada
}

Contexte et problématique: Le Master sciences cliniques infirmières d'Aix-Marseille université existe depuis 2009. Il s'agit d'un des rares dispositifs de formation en sciences infirmières, permettant aux infirmiers de poursuivre sur un niveau Master après le diplôme d'Etat en soins généraux. Il permet également un accès aux études doctorales par la suite.

Son objectif principal est de former des infirmiers de pratique avancée (IPA), exerçant auprès des patients et de leur entourage, en synergie avec les autres membres de l'équipe interdisciplinaire et en adéquation avec les contingences du système de santé. Il s'appuie sur le cadre de référence international, réglementaire et normatif du Conseil International des Infirmières, la pratique avancée étant reconnue dans plusieurs pays.

Description : Le nombre de diplômés s'élève aujourd'hui à 94 (à l'année universitaire 2015-2016). L'implantation de leur rôle dans les milieux cliniques s'organise progressivement, portée notamment par la reconnaissance de la pratique avancée dans la loi de modernisation de notre système de santé du 26 janvier 2016.

Un tronc commun est proposé en première année de Master, puis les étudiants ont le choix entre 4 spécialisations à compter de la rentrée 2017:

- Infirmier coordinateur de parcours complexes de soins ;

- Infirmier de pratique avancée en cancérologie;

- Infirmier de pratique avancée en gérontologie;

- Infirmier de pratique avancée en néphrologie, dialyse et transplantation rénale.

Basés sur une approche par compétence, la formation utilise des méthodes pédagogiques comme l'analyse réflexive sur des situations de soins vécues ou imaginées, le perfectionnement des connaissances, l'observation et l'application clinique durant les stages. Le Master se déroule en alternance d'une activité professionnelle, favorisant le réinvestissement par l'étudiant des compétences acquises durant sa formation.

Le dispositif de formation est engagé dans un processus d'amélioration continue de la qualité, par l'évaluation de la satisfaction des étudiants. Il a fait l'objet d'une évaluation par le Haut Conseil de l'Évaluation de la Recherche et de l'Enseignement Supérieur, qui en a précisé les points forts et les axes d'amélioration. Ces résultats seront présentés lors de la communication orale.

Les perspectives de l'évolution du Master seront discutées, notamment sur sa place dans le paysage national et international de l'offre de formation. Un dispositif d'accompagnement des diplômés du Master à l'implantation des nouveaux rôles de pratique avancée au sein des milieux cliniques est en cours de réflexion.

Mots-clés : Master, sciences infirmières, Aix-Marseille université, dispositif LMD

\section{CO 18}

\section{Parcours universitaires des sages-femmes enseignantes et directrices des écoles de sages-femmes en France: enquête descriptive}

\author{
Christine MORIN ${ }^{1}$, Marie-Christine LEYMARIE ${ }^{2}$ \\ 1 École de sages-femmes, CHU de Bordeaux, Bordeaux, \\ France \\ 2 École de sages-femmes, CHU de Clermont-Ferrand, \\ Clermont-Ferrand, France
}

Contexte: Les exigences académiques pour les sages-femmes enseignantes ne sont pas les mêmes en France que celles d'autres pays européens, comme le Royaume Uni et la Suède par exemple. 
Malgré l'harmonisation européenne des formations de l'enseignement supérieur qui s'est traduite en France par une organisation des études de sages-femmes en cinq ans, la formation des sages-femmes enseignantes n'a pas encore été accompagnée d'exigences universitaires. Il n'existe aucune donnée nationale sur le sujet.

Objectif : Décrire les diplômes universitaires détenus par les sagesfemmes enseignantes et directrices des écoles de sages-femmes en France en 2014.

Méthode: Sondage par questionnaire envoyé par e-mail aux directrices des écoles de sages-femmes pour être rempli par les enseignantes et les directrices.

Participants : Sages-femmes enseignantes et directrices.

Résultats : Les 241 (100\%) sages-femmes enseignantes et directrices en activité dans les écoles de sages-femmes ont participé à l'enquête. La moitié des sages-femmes directrices (17/34) et 88/207 (42,5\%) des sages-femmes enseignantes possédaient un diplôme de Master. Une enseignante et une directrice étaient titulaires d'un doctorat. Au moment de l'étude, 37 (15,3\%) sages-femmes enseignantes ou directrices suivaient un programme de Master et $20(8,3 \%)$ un programme de doctorat dans des disciplines variées (sociologie, santé publique/épidémiologie, philosophie, sciences de l'éducation, droit, management/gestion). Un total de 142 (58,9\%) sages-femmes enseignantes ou directrices avaient validé ou suivaient un programme de Master et $22(9,1 \%)$ étaient titulaires ou suivaient un programme de doctorat.

Discussion : Il s'agit de la première enquête nationale décrivant le niveau académique des sages-femmes enseignantes françaises. Elle s'inscrit dans le cadre des revendications actuelles des sages-femmes françaises pour une meilleure reconnaissance de leur profession et de leur rôle. Cet état des lieux du niveau académique du corps professoral exerçant dans les écoles apporte des éléments utiles pour la demande d'intégration de celles-ci à l'université dans des composantes autonomes et la reconnaissance d'un statut d'enseignant universitaire.

Conclusion : Ce sondage a permis de décrire le parcours académique des sages-femmes enseignantes en France. Le nombre de sagesfemmes enseignantes titulaires d'un doctorat est faible comparé à celui du Royaume Uni ou de la Suède.

Mots-clés : écoles de sages-femmes françaises, sage-femme enseignante, qualifications, université, recherche maïeutique, LMD

\section{CO 19}

\section{Développement d'un programme universitaire pour la supervision clinique: comparaison de deux formats de formation}

\author{
Jehanne DE GRASSET ${ }^{1}$, Jehanne DE GRASSET ${ }^{1}$, Nadia \\ BAJWA2 ${ }^{2}$ Nicole JASTROW ${ }^{3}$, Hélène RICHARD-LEPOURIEL ${ }^{4}$, \\ Marie-Claude AUDETAT ${ }^{5}$, Mathieu NENDAZ ${ }^{6}$ \\ ${ }^{1}$ IMPR, HUG, Genève, Suisse \\ ${ }^{2}$ Pédiatrie, HUG, Genève, Suisse \\ ${ }^{3}$ Gynécologie-obstétrique, HUG, Genève, Suisse
}

${ }^{4}$ Psychiatrie, HUG, Genève, Suisse

${ }^{5}$ UIGP, Université de Genève, Genève, Suisse

${ }^{6}$ UDREM, Université de Genève, Genève, Suisse

Contexte : L'Institut suisse pour la formation médicale post-graduée et continue (ISFM) demande que l'évaluation des performances des médecins en formation post-graduée se fasse en milieu clinique à l'aide des mini-CEX et DOPS. Cependant, les jeunes superviseurs cliniques trouvent souvent difficile de superviser et donner des rétroactions aux médecins en formation post-graduée par manque de compétences pédagogiques, notamment dans les domaines du raisonnement clinique, du professionnalisme, de la communication et des relations interprofessionnelles.

Objectifs : L'objectif de ce projet était d'évaluer si, et en quoi un programme de formation de formateurs à la supervision individualisé serait plus efficace qu'un programme plus traditionnel par ateliers sur les compétences pédagogiques.

Méthode: Nous avons mené une étude d'intervention randomisée contrôlée. Les superviseurs cliniques issus de 5 départements ont été invités à suivre un programme de formation à la supervision sur 6 mois aux hôpitaux universitaires de Genève. La distribution dans l'un des deux formats de formation a été faite de manière randomisée : (1) traditionnel avec 3 ateliers $(7 \mathrm{~h}) ;(2)$ expérimental avec 2 ateliers et $3 \times 1 \mathrm{~h}$ de coaching individualisé basé sur des vidéos de supervision tournées avant l'intervention $(8 \mathrm{~h})$. Les compétences pédagogiques des participants ont été analysées durant 4 séances d'enseignement objectif et structuré (OSTEs) filmées avant et après formation. L'analyse s'est faite à l'aide de grilles développées sur la base des principes pédagogiques relatifs à la rétroaction ainsi qu'à la supervision clinique.

Résultats: $\mathrm{Au}$ total, 86 superviseurs cliniques ont terminé la formation (niveau de participation $95 \%$ ). Les participants des deux groupes ont amélioré leurs compétences pédagogiques à l'issue de la formation. Cependant, l'amélioration a été plus importante dans le groupe « expérimental » pour les dimensions suivantes : Rétroaction : - participation active des apprenants dans la résolution du problème (différence de gain en faveur du groupe expérimental $+0,4$ sur une échelle de Likert 1-5); - utilisation du jeu de rôle $(+0,31)$ et - vérification de la compréhension des éléments discutés durant la rétroaction $(+0,85)$.

Supervision clinique: la différence de gain en faveur du groupe expérimental est globalement moins significative avec toutefois une amélioration pour: - l'exploration des besoins $(+0,69),-$ la stimulation du raisonnement de l'apprenant $(+0,74)$; - le feedback $(+0,41)$ et - le plan d'apprentissage pédagogique $(+0,48)$.

Discussion et conclusion : Former les jeunes superviseurs cliniques aux techniques de supervision et de feedback est efficace selon les deux formats. Une approche individualisée se montre néanmoins supérieure pour quelques éléments spécifiques de la rétroaction et de la supervision clinique. Ces dimensions doivent être encore confirmées en incluant un plus grand nombre de participants, mais elles soulignent d'ores et déjà des possibilités de renforcer la formation des superviseurs sur des aspects déterminants.

Mots-clés : formation, superviseurs clinique, évaluation, dispositif 
$\mathrm{CO} 20$

\section{Méthode d'apprentissage combinée interactive par problèmes cliniques: apprendre par l'erreur collective}

\author{
Antoine AUBRION ${ }^{1,2}$, Anais REICHLING ${ }^{3}$, Marie \\ AUBRION $^{4}$, Vladimir MANSOUR ${ }^{2}$, Eric ROUPIE ${ }^{1}$ \\ ${ }^{1}$ URGENCES - SAMU - SMUR, CHU de Caen, Caen, France \\ ${ }^{2}$ URGENCES - SMUR, CH Robert-Bisson - Lisieux, Lisieux, \\ France \\ ${ }^{3}$ Pharmacie à usage intérieur, Centre hospitalier Robert- \\ Bisson, Lisieux, France \\ ${ }^{4}$ Faculté de médecine de Caen, Université de Caen, Caen, \\ France
}

Contexte: La formation des étudiants paramédicaux est un enjeu crucial des études de santé. Ceux-ci sont en première ligne dans la prise en charge des patients, tant dans leur évaluation à l'admission que dans leur suivi pendant le séjour. Le triage est un processus d'évaluation clinique initiale, qui vise à prendre en charge au mieux chaque patient. C'est un processus de prise de décision complexe permettant d'organiser efficacement le système de soins, lui donnant une place centrale dans le fonctionnement d'un service. Plusieurs échelles de triage ont été conçues comme des systèmes de support de décision pour guider l'infirmière à un choix correct. Ses enjeux d'apprentissage sont donc importants. De nouveaux modèles d'enseignements peuvent nous aider dans cet objectif. Ils préconisent la confrontation, l'expérimentation et le vécu des compétences par l'implication du sujet. L'étudiant doit se poser ses propres questions pour construire ses réponses et pouvoir les intégrer comme fondements de son raisonnement clinique. L'apprentissage par réflexion de groupe est un processus à la fois opposé et complémentaire de la démarche individuelle. La dynamique de groupe appuie la construction de la réflexion individuelle. Les activités récurrentes et la rétroaction offrent également un cadre structurant permettant de rendre plus efficaces l'enseignement et l'apprentissage.

Objectif : Nous avons souhaité tester la combinaison de ces principes généraux d'apprentissage dans cette compétence particulière du triage, en évaluant les buts identifiés de la formation et le ressenti de l'activité pédagogique.

Méthode : Dans le cadre de la formation d'une promotion d'étudiants infirmiers aux principes d'évaluation d'un patient, nous avons mis en place une méthode d'apprentissage en quatre étapes. Dans un premier temps les étudiants assistaient à une présentation théorique des principes du triage, dans l'échelle française puis américaine, qui comportent chacune 5 stades de gravité. Chaque échelle était accompagnée de trois exemples cliniques avec leurs résultats de tris. Dans un deuxième temps les élèves étaient invités à se confronter individuellement à 60 situations cliniques référentes, à classer dans chacune des deux échelles dans un temps limité. Après une période de latence, dans un troisième temps les cas les moins réussis étaient présentés à nouveau aux mêmes étudiants, répartis en plusieurs groupes. Après débat chaque groupe devait se concerter pour présenter un choix de tri. Dans un dernier temps les étudiants étaient confrontés à nouveau à 20 présentations cliniques du panel initial. Les réponses individuelles étaient comparées aux réponses par concertation de groupe. Les réponses individuelles, de la première et la dernière étape, étaient également comparées. Le ressenti des étudiants sur ce mode de formation était recueilli au moyen d'un questionnaire validé. Résultats: Le taux de bonnes réponses des 68 étudiants s'est amélioré entre l'épreuve individuelle (18\%) et la concertation de groupe (37\%); $p<0,01$. Au décours de ces étapes, les réponses individuelles étaient également améliorées (48\% puis $66 \%$; $p<0,01)$. Le ressenti des étudiants sur cette méthode pédagogique en quatre étapes combinées était très favorable.

Conclusion : La combinaison de plusieurs principes pédagogiques nous a permis de développer un dispositif original, répondant aux objectifs fixés d'enseignements et d'apprentissages.

Mots-clés: pédagogie, apprentissage, erreur, formation en santé, échelle de triage

\section{CO 21}

\section{Les récits de situations cliniques authentiques dévoyés}

\section{Daniel BUCHON}

DUMG, Faculté de médecine, Limoges, France

Contexte et problématique: Les récits de situations cliniques authentiques (RSCA) sont des travaux d'écriture, qui doivent mener vers une réflexion personnelle, en démontrant une capacité d'analyse et de synthèse de l'étudiant. La situation vécue est décrite de telle sorte que le contexte de soins primaires, les éléments constitutifs de la complexité sont bien identifiés, la validité scientifique (EBM), la démarche de recherche documentaire nécessaire sont exposés, les compétences mises en œuvre sont clairement identifiées. Ces récits sont intégrés dans le portfolio des internes de DES de médecine générale. À la faculté de médecine de Limoges, un RSCA est demandé par semestre (par stage), contribuant à la validation du DES. Description : L'enseignement facultaire est assuré par les enseignants de médecine générale de la faculté lors de séances de travaux pratiques, d'une durée de quatre heures environ, dans les locaux de la faculté, pour un total de 44 heures environ, par année du DES. Cet enseignement est très interactif, donnant le plus possible la parole aux étudiants. Les thèmes de ces enseignements sont précisés à l'avance, mais restent assez larges, afin de prendre en compte la complexité et la diversité des situations de soins primaires (exemple: «le raisonnement clinique», «l'enfant», «l'adolescent», « la déprescription», «la personne âgée», «le risque cardiovasculaire», etc.). En troisième année, l'enseignement est proposé sous forme de «groupes d'échange de pratique».

Afin de rendre ces travaux pratiques de première et deuxième année le plus actif possible, il a été institué l'utilisation de RSCA à chacune de séances d'enseignement. Deux internes par séance doivent rédiger un récit sur le thème choisi et le présenter en groupe. Une large interprétation du récit est effectuée par le groupe, sous la direction discrète mais active d'un enseignant. L'explicitation du RSCA 
permet de retrouver les problèmes liés aux soins primaires soulevés et de pointer les compétences mobilisées lors de cette situation clinique. Une recherche documentaire sur le thème du récit est habituellement confiée à chacun des deux internes.

Résultats : Cette méthode pédagogique a permis l'implication très active des étudiants dans la préparation et l'animation de ces enseignements facultaires, chaque interne étant mobilisé au moins une fois, au cours des deux premières années du DES. Le groupe est attentif au récit, mais aussi au travail d'interprétation qui l'accompagne. La méthode est plébiscitée par les étudiants, d'autant plus qu'elle permet de comprendre et améliorer la qualité rédactionnelle des RSCA.

Le caractère d'engagement personnel du RSCA est en partie perdu lors de la rédaction commune du récit par les deux internes, mais cette collaboration est le plus souvent fructueuse. Le regard critique des autres membres du groupe est très actif et facilite la compréhension des problèmes posés par un effet analytique et comparatif.

Même si l'utilisation de ces récits ne répond plus à l'objectif initial des RSCA, la qualité pédagogique des enseignements est largement confortée.

Mots-clés : enseignement facultaire, portfolio, écriture

\section{$\mathrm{CO} 22$}

\section{Évolution des séminaires de collaboration interprofessionnelle du pôle santé de l'université Libre de Bruxelles}

\section{Marie BLONDEAU, Marco SCHETGEN}

Faculté de médecine, Université libre de Bruxelles, Bruxelles, Belgique

Contexte et problématique : Depuis 2013, les différentes facultés du pôle santé de l'université Libre de Bruxelles ont mis sur pied des séminaires de formation à la pratique interprofessionnelle. Les étudiants concernés sont ceux qui terminent leurs études en médecine, pharmacie, kinésithérapie, ostéopathie et santé publique, auxquels se sont ajoutés en 2014 les étudiants en soins infirmiers de la HELB. Au total, 600 étudiants sont concernés.

Description : Chaque étudiant participe à 2 séminaires organisés en sous-groupes pluridisciplinaires. Les objectifs visés sont : connaître le travail effectué par les autres professionnels, comprendre l'intérêt de chaque discipline dans le cadre d'une prise en charge interdisciplinaire et communiquer entre acteurs des différentes disciplines. Les étudiants organisés en sous-groupes multidisciplinaires de 8 ou 9 échangent autour de cas cliniques. Lors du premier séminaire, ils sont amenés à expliquer aux autres participants les spécificités de leur métier au travers du cas étudié. Le second séminaire exige de chaque groupe d'élaborer de manière collaborative un projet de prise en charge du patient. Chacun des séminaires se termine par une séance plénière qui vise à synthétiser les apprentissages réalisés.

Évaluation : Chaque année le dispositif est évalué par un pré-test et un post-test et par un questionnaire de satisfaction. Voici en quelques lignes le bilan de ces quatre dernières années, ce qui a bien fonctionné et ce que nous pouvons encore améliorer.
Ce qui fonctionne bien:

- l'intégration aux cursus des étudiants dans des unités d'enseignement existantes ;

- le travail en deux étapes, avec des objectifs plus complexes (connaître les autres, puis collaborer);

- l'animation par des enseignants issus des différentes disciplines représentées ;

- la création d'un guide à destination des étudiants reprenant les consignes, le calendrier...;

- l'utilisation de la plateforme d'apprentissage en ligne pour communiquer avec l'ensemble des étudiants et évaluer le dispositif;

- l'organisation d'une séance de préparation pour les étudiants en santé publique;

- le focus sur les rôles et fonctions, puis sur la collaboration lors des synthèses (et non la résolution clinique de la vignette);

- l'intégration de nouveaux participants, en sciences infirmières, et bientôt assistants sociaux;

- la participation de quelques patients partenaires lors du deuxième séminaire.

Ce qui peut encore être amélioré :

- l'évaluation des apprentissages via le pré-test et post-test;

- l'évaluation de l'impact des séminaires sur le développement des compétences (long terme);

- les outils, traces et pistes concrètes laissées aux étudiants ;

- la mise en place d'un réel partenariat avec des patients formateurs, sélectionnés et formés.

Résultats : Une formation à la collaboration interprofessionnelle est de plus en plus indispensable pour les futurs acteurs de notre système de santé. Le dispositif mis en place, évalué chaque année par les différents acteurs, a beaucoup évolué en 4 ans. Les séminaires atteignent un taux élevé de satisfaction. Malgré ça, le corps enseignant souhaite encore améliorer les séminaires en y développant le partenariat patient (Pomey, 2015). Il s'agira donc, dès l'année prochaine, de recruter et former des patients formateurs qui seront impliqués dans l'entièreté du dispositif (préparation, animation et évaluation).

Mots-clés : collaboration interprofessionnelle, communication, patient partenaire, dispositif de formation

$\mathrm{CO} 23$

Accompagner le développement professionnel d'étudiants en psychologie: présentation d'un dispositif mis en place à l'université de Liège

\section{Stéphanie PETERS, Trecy MARTINEZ-PEREZ, Anne-Marie ETIENNE}

Faculté de psychologie, logopédie et sciences de l'éducation, Université de Liège, Liège, Belgique

Contexte et problématique : Depuis quelques années, l'American Psychological Association (APA) suggère d'introduire dans les 
cursus de formation en psychologie des cours qui permettent aux étudiants d'anticiper la diversité des opportunités professionnelles qui s'offrent à eux, d'une part, et de mieux s'approprier les champs d'action, les compétences, les rôles et limites de leur future profession, d'autre part (APA, 2013).

Description : Cette contribution s'inscrit dans cette démarche. Elle présente un dispositif d'accompagnement du développement professionnel d'étudiants en psychologie de l'Université de Liège (Belgique). Ce dispositif est proposé durant les trois premières années de la formation (Bachelier). Sa conception a été principalement guidée par un souci de triple concordance (Leclercq et Denis, 1998), c'est-à-dire une adéquation optimale entre les objectifs d'apprentissage, les activités d'apprentissage, et l'évaluation de ces apprentissages.

Ainsi, l'objectif est d'accompagner les étudiants dans leur développement professionnel en suscitant une réflexion dynamique. Les activités d'apprentissage sont conçues de manière à accroître progressivement l'implication de l'étudiant dans son développement professionnel. Le dispositif se décline de la sorte :

- première année: le cours vise à (1) découvrir un éventail de réalités professionnelles du psychologue, (2) initier une réflexion sur des liens entre les cours et la future pratique, (3) se rencontrer, créer du lien. Pour ce faire, les étudiants assistent à des conférences métiers durant lesquelles des professionnels témoignent de leur travail au quotidien et échangent avec les étudiants. Un journal de bord permet aux étudiants de consigner leurs réflexions tout au long de l'année. En outre, un séminaire en petits groupes est proposé pour les aider à mener une réflexion critique ;

- deuxième année : le cours vise à (1) élargir l'éventail de réalités professionnelles du psychologue, (2) approfondir les liens entre les cours et la future pratique, (3) créer du lien et travailler ensemble. Pour ce faire, les étudiants doivent, en groupe, mener à bien un projet de découverte de la profession qui passe par la rencontre avec un professionnel sur son lieu de travail. En outre, ce projet doit être partagé avec les autres étudiants par le biais d'une capsule vidéo de 3 minutes;

- troisième année: les étudiants ont la possibilité de faire l'expérience de réalités professionnelles, en s'inscrivant dans un projet de recherche et/ou une intervention en cours au sein d'une des unités de la faculté.

Résultats : L'évaluation, pour les trois années, témoigne quant à elle d'un véritable renversement de la preuve, car ce sont les étudiants qui doivent montrer ce qu'ils ont appris par le biais d'une synthèse écrite. L'enseignant note l'étudiant sur son niveau de réflexivité autour des apprentissages (Jorro, 2005), et non sur le contenu de ces apprentissages. Une procédure d'évaluation du dispositif pour les deux premières années a été mise en place. Elle porte sur la satisfaction des étudiants, l'organisation pédagogique du cours et les apprentissages réalisés par les étudiants. Ces données seront présentées et discutées dans le cadre de cette communication.

Mots-clés : psychologues, développement professionnel, réflexivité
$\mathrm{CO} 24$

\section{Une formation continue innovante de l'université de Genève: le Certificat (CAS) de formateur dans les sciences de la santé}

\section{Anne BAROFFIO}

Unité de développement et de recherche en éducation médicale, Faculté de médecine, Université de Genève, Genève, Suisse

Contexte et problématique : L'enseignement de la médecine et des sciences de la santé en général, ainsi que les besoins de la société, ne cessent d'évoluer. Un article fondateur publié en 2010 dans le Lancet [1] est venu rappeler que même si la formation des médecins a énormément évolué au cours du $20^{\mathrm{e}}$ siècle, elle est aujourd'hui inadaptée aux défis du $21^{\mathrm{e}}$ siècle. Une commission de 20 leaders professionnels et académiques de différents pays ont planché sur une vision et une stratégie commune pour la formation des professionnels de la santé. Ils ont proposé 9 réformes dont 6 concernent la formation : (a) Adopter des programmes d'études axés sur l'acquisition de compétences qui sont capables de répondre à des évolutions rapides ; (b) Promouvoir la formation interprofessionnelle qui permet d'améliorer les relations collaboratives et le travail d'équipe; (c) Exploiter la puissance des technologies de l'information pour l'apprentissage à distance, l'apprentissage collaboratif, les simulations, etc.; (d) Exploiter les ressources globales tout en les partageant et les adaptant aux contextes locaux; (e) Renforcer et développer les ressources éducatives qui sont les instruments nécessaires pour développer les compétences; (f) Promouvoir un nouveau professionnalisme, incluant un ensemble de valeurs et de comportements communs aux professionnels de santé, et permettant de développer leurs rôles d'agents de changement, de gestionnaires des ressources compétents et de promoteurs de politiques fondées sur des preuves. Description: L'université de Genève, conjointement avec le département de médecine de l'université de Fribourg et en partenariat avec la Haute École de Santé des Hautes Écoles de Suisse occidentale développe un nouveau CAS qui s'adresse aux formateurs des professions de la santé. Les programmes CAS («certificate of advanced studies»/certificat de formation continue) sont des diplômes d'études supérieures offrant une formation diplômante qui permet d'acquérir une qualification supplémentaire dans sa profession. Ce CAS novateur répond à la plupart des défis soulevés dans l'article du Lancet: il est basé sur les compétences, est élaboré par des enseignants de différentes professions de la santé et s'adresse à un public interprofessionnel, donne l'occasion de travailler en équipe, favorise une part de travail à distance en utilisant les technologies de l'information, offre une formation sur les pratiques simulées, permet aux participants d'appliquer ce qu'ils apprennent à un projet personnel qu'ils seront amenés à développer dans leur contexte local, et finalement est organisé autour d'un fil rouge qui est le professionnalisme.

La communication décrira les principes pédagogiques de cette formation, sa structure modulaire, l'articulation entre travail 
présentiel et à distance, ainsi que les compétences qui seront développées par les participants.

\section{Référence}

[1] Frenk et al. Health professionals for a new century: transforming education to strengthen health systems in an interdependent world. Lancet 2010;376:1923-58.

Mots-clés : formateur dans les sciences de la santé, formation continue

CO 25

\section{Un «Massive Open Online Course» (MOOC) sur des travaux pratiques en histologie: le grand écart entre son intégration dans l'enseignement universitaire et l'intérêt du grand public}

Sylvie MULTON ${ }^{1}$, Laurence PESESSE ${ }^{1}$, Alodie WEATHERSPOON ${ }^{1}$, Sandra FLORQUIN ${ }^{1}$, Jean-François VAN DE POEL ${ }^{2}$, Pierre MARTIN², Grégoire VINCKE ${ }^{2}$, Dominique VERPOORTEN ${ }^{2}$, Quatresooz PASCALE ${ }^{1}$, Pierre BONNET $^{3}$, Valérie DEFAWEUX ${ }^{4}$

${ }^{1}$ Faculté de médecine, histologie, Université de Liège, Liège, Belgique

2 IFRES, Université de Liège, Liège, Belgique

${ }^{3}$ Faculté de médecine, anatomie, Université de Liège, Liège, Belgique

4 Faculté de médecine, histologie anatomie, Université de Liège, Liège, Belgique

Contexte et problématique: L'acronyme anglophone MOOC désigne les «Massive Open Online Courses» que l'on pourrait traduire par «formations en ligne massives ouvertes à tous ». Nés aux États-Unis avec un programme sur l'intelligence artificielle dispensé par l'université de Stanford, ces cours en ligne accessibles gratuitement font miroiter la possibilité d'une démocratisation maximale des savoirs. À ce titre, les MOOC bénéficient des innovations issues du Web 2.0 : ils tirent le meilleur de la rencontre entre les nouveaux usages induits par les réseaux sociaux et les apports de la pédagogique active. Description: Ces cinq dernières années, nous avons dispensé les travaux pratiques en histologie aux 500 étudiants de première année d'étude de la faculté de médecine de l'université de Liège par une méthodologie hybride alliant e-learning et présentiel (Multon, 2015). Forte de cette expérience réussie, notre équipe a réalisé un MOOC faisant partie intégrante du cursus de nos étudiants et qui répond à la volonté de partager nos ressources numériques avec un public plus large. Ce MOOC, intitulé «Introduction à l'histologie, exploration des tissus du corps humain », est dédié à l'étude des 5 familles de tissus biologiques. Deux parcours pédagogiques permettent d'approfondir différemment la matière et ainsi de mieux correspondre aux objectifs ou motivations de chacun. La première session, hébergée sur la plateforme FUN (France Université Numérique), s'est déroulée en 6 semaines à partir de février 2017. Au travers de vidéos explicatives contenant des dessins construits au fur et à mesure et des liens avec l'anatomie suivie d'activités interactives telles que la manipulation d'un microscope virtuel, de nombreux quiz et d'activités réalisées avec les pairs, les participants seront capables de reconnaître les différents types cellulaires et tous les éléments qui les entourent afin de comprendre comment ils s'organisent entre eux pour former des tissus aux fonctions spécifiques. À l'issu de chaque module, les apprenants ont la possibilité de réaliser des activités certificatives. L'obtention de $70 \%$ à chacune donne lieu à une attestation de suivi avec succès du MOOC.

Résultats : Notre MOOC a attiré 5250 personnes issues de 82 pays. Un sondage à l'inscription $(n=841)$ indique que $42 \%$ sont des étudiants et $41 \%$ ont une activité professionnelle. Un forum actif a permis de nombreux échanges entre apprenants ou avec l'équipe pédagogique (170 fils de discussion). De nombreux participants ont souligné les aspects didactique, accessible, dynamique et captivant du MOOC. Nos premières données montrent que $32 \%$ des apprenants ont utilisé le microscope virtuel et que $20 \%$ se sont engagés dans les activités certificatives, la moitié d'entre eux les ayant réussies.

Nos étudiants ont apprécié la possibilité de travailler où et quand ils le souhaitaient tout en étant guidés par la structure des différents modules. Un sondage final est actuellement analysé, il permettra d'étudier leur ressenti sur les différentes composantes de l'expérience d'apprentissage. Dix pour cent des participants ont obtenu l'attestation finale, la moyenne se situant généralement autour de $5 \%$ pour les autres MOOC (Oliveri, 2016).

Conclusion: Notre MOOC a réussi le pari d'intéresser un vaste public en étant intégré dans un enseignement universitaire.

Mots-clés : histologie, MOOC, microscopie virtuelle

\section{$\mathrm{CO} 26$}

\section{La sémiologie en 11 signes}

\section{Iris NAUDIN}

\section{Chirurgie vasculaire, Hôpital Édouard-Herriot, Lyon, France}

Contexte et problématique : L'apprentissage de l'examen clinique d'un patient est une étape fondamentale dans le cursus d'un étudiant en médecine. Il n'est cependant pas toujours facile à acquérir du fait d'un manque de temps et de ressources des services hospitaliers où les étudiants se retrouvent de plus en plus nombreux. Ce projet est basé sur un article paru dans «La revue de médecine interne» en 2014 qui montre que les étudiants de DCEM4 ont des lacunes dans la pratique clinique et la connaissance des signes séméiologiques fondamentaux. Description : Onze signes cliniques ont été retenus dans cette étude. Il s'agit de: palpation des pouls périphériques aux membres inférieurs, évaluation des signes de lutte et d'épuisement respiratoire, palpation abdominale, interrogatoire d'une douleur abdominale, recherche d'un syndrome cérébelleux statique, percussion des réflexes ostéo-tendineux, évaluation des signes d'insuffisance hépato-cellulaire, palpation des aires ganglionnaires superficielles, 
évaluation des signes d'insuffisance cardiaque droite, évaluation de l'attention. Ils ont été sélectionnés par 4 médecins internistes des universités Pierre-Marie-Curie et Paris Descartes impliqués dans l'enseignement en fonction des objectifs du stage de séméiologie clinique de deuxième année du premier cycle des études médicales. Les résultats montrent qu'un faible pourcentage de ces étudiants de DCEM4 sait rechercher ces tableaux cliniques pourtant basiques. Nous proposons un projet de courts films explicatifs basés sur ces onze signes sémiologiques, mis à la disposition des étudiants en médecine sur le site Internet de la faculté de médecine de Lyon et sur une plateforme de partage de vidéos en ligne.

Les vidéos font intervenir un médecin spécialiste qui recherche le symptôme/syndrome sur un patient anonyme présentant les signes d'intérêt. La vidéo est agrémentée de schémas animés en infographie pour expliquer la physiopathologie du signe. L'intérêt du signe, sa spécificité et sa sensibilité sont aussi discutés. Un QCM de 5 questions à la fin de la vidéo permet une auto-évaluation de l'étudiant sur les caractéristiques majeures du signe clinique concerné.

Discussion : Nous voulons ainsi faciliter l'apprentissage séméiologique des étudiants en médecine à travers un support numérique facile à manier et à comprendre. Il pourra être utilisé par les enseignants en travaux pratiques ou en stage hospitalier.

Mots-clés : sémiologie, premier cycle des études médicales, vidéo, examen clinique

$\mathrm{CO} 27$

\section{Tensions identitaires et conflits professionnels : implications pour la formation médicale post-grade}

\author{
Naike BOCHATAY ${ }^{1}$, Nadia BAJWA ${ }^{1,2}$, Katherine \\ $B_{\text {BLNDON }}^{3}$, Noelle Junod PERRON ${ }^{1,4}$, Stephane \\ CULLATI ${ }^{5}$, Mathieu NENDAZ ${ }^{1}$
}

${ }^{1}$ Unité de développement et recherche en éducation médicale, Faculté de médecine, Université de Genève, Genève, Suisse

${ }^{2}$ Pédiatrie, Hôpitaux Universitaires de Genève, Genève, Suisse

3 Direction médicale, Hôpitaux universitaires de Genève, Genève, Suisse

${ }^{4}$ Institut de médecine de premier recours, Hôpitaux universitaires de Genève, Genève, Suisse

5 Qualité des soins, Hôpitaux universitaires de Genève, Genève, Suisse

Contexte: La profession médicale s'est historiquement construite autour de valeurs telles que le dévouement aux patients, l'altruisme, et le désir d'aider et soulager, avec une activité impliquant également une forte composante relationnelle. Néanmoins, de nos jours, les médecins en formation post-grade doivent également gérer des tâches, administratives notamment, d'une complexité croissante dans un temps de travail restreint. De plus, ils doivent satisfaire aux exigences propres à leur formation et leur carrière. Ces éléments peuvent être perçus comme des freins à l'expression des valeurs de base liées à l'identité professionnelle et peuvent générer des tensions dans les équipes médico-soignantes.

Objectif: Nous avons cherché à comprendre quels aspects de l'identité professionnelle de médecins hospitaliers étaient touchés par les tensions au travail, et ce, dans le but d'identifier des éléments permettant d'améliorer les conditions de la formation post-grade.

Méthode: Dans le cadre d'une étude sur les expériences de conflits vécues par les professionnels de la santé, nous avons réalisé des entretiens semi-directifs avec des médecins internes et chefs de clinique travaillant dans différents services des hôpitaux universitaires de Genève. L'analyse inductive de nos entretiens nous a amenés à constater que de nombreux aspects identitaires étaient impliqués dans les situations de conflits rapportées, soit comme source, soit comme conséquence des conflits. Ces aspects ont été approfondis à travers une analyse de contenu.

Résultats : Parmi les 43 médecins ayant participé à notre étude, 16 ont rapporté des situations de conflits impliquant les valeurs à la base de la profession médicale. Ces situations, vécues par les jeunes participants ou observées par les plus expérimentés, indiquaient que le début de la carrière du médecin représente une période critique. L'entrée dans le monde professionnel des médecins était, selon eux, caractérisée par des aspects identitaires tels que l'incertitude quant à leur avenir, le risque d'être mal considérés par leurs supérieurs (ce qu'ils percevaient comme pouvant nuire à leur formation et leur carrière), les tensions entre les exigences de formation et les conditions de travail, et la difficulté à maintenir un équilibre entre vie privée et vie professionnelle. Les conflits rapportés avaient amené certains répondants à se sentir humiliés, épuisés, ou à se distancer de leur travail. Ceci pouvait les amener à se détacher de leurs valeurs, parfois jusqu'au cynisme. Ces tensions pouvaient également étioler la confiance entre les membres des équipes médico-soignantes.

Discussion et conclusion: Nous avons identifié certains aspects identitaires, sources de conflits et de frustrations chez les jeunes médecins, qui pouvaient affecter leur bien-être et la qualité de leur travail. Les conflits ainsi générés traduisaient un décalage entre l'idéal de pratique des répondants et leur contexte de travail. Nos résultats soulignent l'importance de soutenir les médecins au début de leur formation post-grade et de se soucier des conditions de formation.

Mots-clés : formation médicale post-grade, conditions de formation, identité professionnelle

\section{$\mathrm{CO} 28$}

\section{La réalité virtuelle: un outil d'enseignement universitaire pour les modèles de croyances liés à la santé, à destination de futurs psychologues et d'acteurs en santé publique}

Anne-Marie ETIENNE

Psychologie de la santé, Université de Liège, Liège, Belgique 
Contexte et problématique : L'université a un rôle à jouer dans la transition vers la société numérique. Le support numérique permet un accès aux savoirs moins contraint temporellement et géographiquement (Goastellec, 2014) et en même temps, il est porteur d'une culture du foisonnement et de l'instantané aux antipodes de la tradition académique (Loisy, 2014). En conséquence, un des enjeux d'une transition numérique réussie réside dans la transformation du métier de l'enseignant, tout en laissant une belle place à la créativité (Psotka, 2013).

Les référentiels de compétences définissent ce qui doit être appris aux différents moments du parcours universitaire et le niveau de maîtrise à atteindre. Ces compétences supposent tant l'éducation « au » numérique que «par » le numérique (Merchant et al., 2014; Pacte d'excellence, 2017). C'est pourquoi à l'université de Liège (Belgique), un nouveau projet transversal d'enseignement par le numérique est en cours de construction; un de ses objectifs sera de faciliter la progression des compétences et apprentissages critiques des étudiants pendant leur cursus universitaire: du Bloc $1 \mathrm{du}$ Bachelier au Bloc 2 du Master.

Description : Le cours de psychologie de la santé constituera un des pilotes de cette nouvelle façon d'enseigner. Il s'agira d'intégrer la réalité virtuelle comme outil d'aide à la compréhension. Les environnements virtuels permettront d'incorporer des concepts abstraits (les attitudes, les normes subjectives, etc.) dans des expériences concrètes (l'adoption d'un comportement de santé par l'usager).

Pour illustrer ces concepts abstraits, nous utiliserons les principes du dispositif de l'échafaudage (scaffolding). La compétence de l'étudiant ira en augmentant: nous partirons d'environnements virtuels «secure» et généraux vers des environnements plus complexes : par exemple mettre en place un comportement de santé, seul ou en présence d'autrui, à son domicile puis dans un restaurant; ou encore en état de «bonne» santé versus malade.

Les étudiants (psychologues et acteurs en santé publique) recevront les concepts associés aux modèles de santé à l'intérieur d'un module de cours qui comprendra des exposés classiques, un chapitre de livre, des exercices et plusieurs séances d'immersion en réalité virtuelle en grand auditoire. Il y aura uniquement trois temps d'évaluation : avant de commencer ce dispositif, une semaine après avoir terminé le module et au cours de la session d'examen (Merchant, 2014). Les étudiants $(n= \pm 200)$ répondront à plusieurs questionnaires : sentiment de présence, connaissances théoriques et pratiques à propos des concepts ainsi que les compétences acquises (cf. Référentiel de psychologie / santé publique),

Discussion : Nous discuterons de la plus-value de ce dispositif pour l'enseignant. Ainsi, selon Quales et al. (2009), les résultats attendus pourraient être que la fusion des espaces réels et virtuels devraient offrir « un niveau unique d'échafaudages éducatifs », ainsi qu'un transfert d'apprentissage amélioré des domaines abstraits et concrets. Nos attentes vont dans ce sens et même nous pensons que la réalité virtuelle peut ne pas être juste efficace, elle peut donner envie de poursuivre l'apprentissage de manière informelle et en dehors de l'université.

Mots-clés : réalité virtuelle, enseignement, psychologie de la santé, santé publique

\section{CO 29}

\section{Un curriculum orienté vers le professionnalisme. Défis d'une faculté de médecine algérienne}

\author{
Mohamed Ridha GUEDJATI, Boubaker KERMICHE, Naouel \\ CHIBOUT
}

Faculté de médecine de Batna, Université Batna 2, Batna, Algérie

Contexte : Les facultés de médecine algériennes suivaient l'orientation innovante des années 1990. «L'étudiant doit être mis dans son environnement pour favoriser son apprentissage intégré de l'éthique, de la déontologie, de la psychologie médicale et de l'économie de santé ». Cette orientation s'intègre dans les dimensions du professionnalisme. Les réformes des années 1992-1995 ont conduit des programmes disciplinaires modulaires imbriqués dans un cursus déjà alourdi par des enseignements spécialisés.

Objectif : Une réflexion critique du cursus de médecine générale a été engagée afin d'identifier les acquis et les insuffisances des réformes (1992-1995) concernant le professionnalisme.

Méthode : Quatre groupes focalisés se sont attachés à identifier les traits du professionnalisme dans le cursus des études de médecine. Les recommandations des $15^{\mathrm{e}}$ Journées de la Conférence Internationale des Doyens des Facultés de médecine d'expression française (CIDMEF) Nancy 2003 nous ont servi de canevas de travail. Les onze recommandations ont été observées lors de la réflexion critique des cinq phases des programmes portant sur le professionnalisme (conception, planification, mise en œuvre, développement et évaluation). Un rapport a été soumis à la conférence des doyens des facultés de médecine algériennes.

Résultats et discussion: Lors de la conception des programmes portant sur le professionnalisme, les facultés de médecine suivaient l'évolution des tendances pédagogiques; ainsi l'orientation intégrée relevant du courant constructiviste des années 1990 se faisant sentir. Le professionnalise était une priorité au début des réformes par l'introduction des dimensions comme l'éthique médicale, la déontologie, la psychologie et l'économie de santé. Le manque de clarté dans la conception des programmes, a conduit de manière automatique à des insuffisances dans les phases de planification et mise en œuvre. Des questions fondamentales telles que «Que mettre dans le programme du professionnalisme?» en faveur de la relation médecin-patient et médecin avec son entourage. Ou bien «Comment mettre en place le programme intégré de l'éthique médicale?» en utilisant un carnet de stage avec la possibilité de portfolio et/ou un apprentissage au raisonnement éthique (ARE). Ces questions ont été peu envisagées lors des phases planification et mise en œuvre des programmes.

Conclusion : L'introduction d'un programme portant sur le professionnalisme était une priorité lors des réformes (1992-1995) du cursus de médecine générale. Les phases planification et mise en œuvre du programme portant sur le professionnalisme, ont été peu observées. 
Mots-clés: professionnalisme, un apprentissage intégré, ARE, planification

$\mathrm{CO} 30$

\section{Stratégies éducationnelles vers une approche par compétence utilisant une plateforme e-learning et le problem-based learning d'un Master en pratique infirmière avancée en onco-hématologie}

Idrissa DIAWARA ${ }^{1}$, Lamia SLIMANI ${ }^{1}$, Ali JAFRII ${ }^{1}$ Rachid GOUIFRANE ${ }^{2}$, Jihane TOUGHZA', Nabil ISMAILI', Wafaa KAIKANI ${ }^{3}$, Fatima DEHBI ${ }^{1}$

1 Faculté des sciences et techniques de santé, Université Mohammed VI des sciences de la santé, Casablanca, Maroc

2 Faculté des sciences Ben M'Sik, Université Hassan II, Casablanca, Maroc

3 Laboratoire national de référence (LNR), Université Mohammed VI des sciences de la santé, Casablanca, Maroc

Contexte: Les professions de santé notamment en sciences infirmières et techniques de santé constituent les métiers de l'avenir. Objectif : L'objectif de cette expérimentation pédagogique est de développer un Master novateur en pratique infirmière avancée en onco-hématologie à la faculté des sciences et techniques de santé de l'Université Mohammed VI des sciences de la santé (UM6SS) de Casablanca, Maroc, en collaboration avec le College of Nursing de l'Université de Tennessee aux USA.

Méthode: Le master développé est constitué de 4 semestres dont deux semestres de formation en sciences fondamentales, un semestre de spécialisation en pratique infirmière avancée en onco-hématogie et un semestre de stage professionnel. L'organisation de ce master à l'UM6SS tient compte de la plateforme blackboard learn et le International Medical Simulation Center (IMSC) disponibles à l'université. Le curriculum du master a été élaboré selon l'approche par compétence utilisant le problem-based learning, le e-learning et la simulation médicale comme méthodes d'apprentissage. L'élaboration de la stratégie éducationnelle était basée sur :

- Élaboration d'un tableau de spécification définissant les référentiels de compétence décomposés en objectifs pédagogiques d'apprentissage fondés sur le raisonnement clinique, les gestes et procédures et la relation soignant-soigné. Pour chaque objectif pédagogique, les différents types d'activité d'apprentissage (AP) ont été définis. Les AP définis étaient :

- Pour le e-learning sur blackboard learn: exposé illustré, quiz, exercice, didacticiel, film, simulation médicale virtuelle, document pdf,

- Pour le présentiel : étude de cas, jeu de rôle, travaux de petit groupe en atelier et séminaire, simulation médicale en présentiel. Les cours en présentiel étaient organisés en raison de 1 weekend sur 2;
- Élaboration d'indicateur de suivi pour l'évaluation de la stratégie éducationnelle. Les indicateurs choisis étaient: le nombre de consultation et le temps d'accès aux contenus digitalisés.

Résultats: Dans cette expérimentation pédagogique, tous les modules relatifs aux connaissances fondamentales ont été digitalisés en e-learning sur blackboard learn. Les objectifs en savoir-faire relatifs aux gestes et procédures ont fait l'objet d'apprentissage par simulation médicale, des études de cas en présentiel sous forme de problem-based learning et des autoformations en e-learning tutoré. Quant aux objectifs en savoir être, relatif au résonnement clinique, les activités étaient de type e-learning avec didacticiel présentant des pratiques infirmières avancées. Le volume horaire des modules des semestres 1 et 2 était comme suit : $30 \%$ du contenu digitalisé en elearning, $30 \%$ de présentiel sous forme de travaux dirigés, travaux pratiques et de simulation et $30 \%$ pour l'autoformation et $10 \%$ pour l'évaluation. Quant aux modules du semestre 3, seules les compétences métacognitives et de créativité ont été retenues selon le même rapport que les semestres précédents.

Conclusion: Cette stratégie a permis de tenir compte de tous les problèmes de santé relatifs à la pratique infirmière avancée en oncohématologie, de les prioriser et de les enseigner en e-learning et selon une approche participative en présentiel sur le problem-based learning.

Mots-clés : onco-hématologie, blackboard learn, problem-based learning, Master en sciences infirmières, E-learning

\section{CO 31}

\section{La responsabilité de la pédagogie inversée dans les bons résultats à $\mathrm{l}^{\prime} \mathrm{ECNi}$}

\section{Jean-Jacques MOREAU, P.-Y. ROBERT, I. PHILIPPONNET, S. CHALIFOUR, A. BELLATI, D. VALLEIX}

Faculté de médecine, Département universitaire d'enseignement numérique en santé 2, rue du Docteur-Marcland, 87025 Limoges France

Contexte et problématique : L'examen national classant informatisé (ECNi) ponctuant la fin des études médicales a profondément modifié l'organisation pédagogique des facultés de médecine. Le classement obtenu par l'étudiant est déterminant pour le choix de sa future spécialité et le lieu de sa formation professionnelle. Une réforme, initiée suite aux bouleversements docimologiques créés par l'ECNi, a modifié les formes d'enseignement de la faculté de médecine de Limoges en s'appuyant sur la pédagogie inversée.

Description : La réforme pédagogique, débutée en 2013, avait pour but d'obtenir un meilleur classement des étudiants de Limoges à l'ECNi.

Les 3 dernières années des études médicales ont été concernées par cette réforme pédagogique qui a comporté 3 « tours » d'enseignement de difficulté croissante.

Le $1^{\text {er }}$ tour sous la forme d'un tutorat faisait appel à la pédagogie inversée : 
- Les cours sont enregistrés sur une plateforme (MOODLE) sous forme de diaporamas commentés que les étudiants devaient avoir appris avant la séance de tutorat;

- Le tutorat est réalisé en présentiel et comporte un exercice sur tablette, une correction, une analyse des statistiques de l'exercice en temps réel, un feedback;

- La banque locale et la banque nationale d'exercices et d'examens (SIDES) où sont stockées les évaluations produites en local et au niveau national.

Le $2^{\mathrm{e}}$ tour, comporte des exercices à distance et une correction en présentiel.

Le $3^{\mathrm{e}}$ tour se fait complètement à distance avec exercices, corrections et commentaires des enseignants.

La pédagogie inversée a visé à développer l'autonomie et la responsabilité de l'étudiant dans son apprentissage. Dans ce contexte, le $3^{\text {e }}$ tour d'entraînement est significatif, car programmé avec des critères particuliers: hebdomadaire, facultatif, aucune évaluation sommative, aucune validation, des dossiers transversaux, des exercices et corrections délocalisées.

Cette forme d'enseignement a permis d'améliorer de $25 \%$ le taux de réussite aux examens facultaires en première session depuis 2014 . Pour l'ECNi, la faculté de médecine de Limoges réalise son meilleur score depuis 2004 en gagnant 7 places au classement des facultés en 2016 et 15 places en 2017 par rapport à 2015 .

Discussion : Des ajustements ont été nécessaires en cours de réforme pour répondre aux difficultés rencontrées par les étudiants: formations de formateurs, amélioration de la forme des diaporamas, accès au PDF des diaporama, etc. Cette progression dans l'utilisation du numérique est nécessaire ce qui évite les abandons d'étudiants, le renoncement des enseignants réfractaires au changement et aux nouvelles technologies et la surcharge d'activité des administratifs. La mise en place d'un tel modèle nécessite un soutien des autorités facultaires, la création d'un environnement numérique (plateforme pédagogique, studio d'enregistrement, e-learning) et l'organisation de formations spécifiques.

En 2016, l'intérêt suscité et la réussite aux examens ont permis de structurer un département d'enseignement numérique en santé (DUENES) pour répondre aux demandes exponentielles de l'utilisation des outils numériques dans l'enseignement supérieur.

$\mathrm{CO} 32$

Développement majeur de la formation interprofessionnelle à Genève : étudiants de la santé, en médecine humaine, dentaire, en pharmacie et en santé communautaire tous ensemble

Petra MÈCHE, Claire Lise MEYENBERG, Liliane DOUCHAMPS, Michael ATTIAS, Adela IBRAHIMOVIC, Carolyn WYNDHAM-WHITE, Anna PILLARD, Emilien JEANNOT

Haute école de santé, Genève, Suisse
Contexte et problématique : Depuis 2012, la Haute école de santé de Genève offre un curriculum interprofessionnel impliquant ses 750 étudiants Bachelor de 5 filières différentes (nutrition et diététique, physiothérapie, sage-femme, soins infirmiers et technique en radiologie médicale). Une équipe pédagogique interprofessionnelle assure le suivi des étudiants transversalement sur l'ensemble des trois ans de formation. Basé sur les guidelines et la définition internationale de l'interprofessionalité selon le CAIPE, l'objectif principal est : « ... de réunir 2 professions ou plus, dans un processus d'apprentissage conjoint... ». Le programme est organisé autour d'une vision et des missions fortes, décrites et inspirées d'auteurs tels que Frenck et al. (2010) et 1'OMS (2010).

Description : Dès 2018, 300 étudiants en médecine et pharmacie (niveau Bachelor puis Master) rejoindront ce programme décomposé en différentes modalités pédagogiques selon les années d'études. Les apprentissages visés et partagés entre la Haute école de santé et les facultés de médecine et de pharmacie poursuivent trois axes majeurs : créer des synergies de compétences interprofessionnelles, métamorphoser une culture professionnelle cloisonnée et faciliter une pratique collaborative en santé-social à Genève. Le but étant, de former de futurs professionnels capables d'interagir et de mobiliser leurs ressources individuelles et collectives pour faire face à des situations de plus en plus complexes, souvent chroniques, sur le plan individuel ou familial, en intra ou extrahospitalier. À travers des mises en situations virtuelles ou réelles, les étudiants développeront leurs outils et une intelligence interprofessionnelle incluant les patients/ clients comme partenaires. Pour structurer la communication, nous utilisons TeamStepps ${ }^{\circledR}$, en particulier lors de pratiques simulées au centre interprofessionnel de simulation.

Le programme 2017-2018 va être le théâtre d'une recomposition majeure en janvier 2018. Durant une semaine, nous allons réunir pas moins de 600 étudiants entre la $1^{\text {re }}$ année Bachelor en santé et la $2^{\mathrm{e}}$ année de médecine humaine, dentaire et pharmacie. Une participation des assistantes en santé et soins communautaires est également envisagée. Pour entrer en relation et réaliser des travaux d'équipes interprofessionnels, les journées débuteront par une séance plénière thématique et la projection d'un scénario patient-centré filmé. Les thèmes de santé choisis sont abordables en début de formation: maladies infectieuses et vaccination, prévention de la dépression et du suicide chez le jeune adulte, prévention du cancer du sein, propagation des infections sexuellement transmissibles et stressépuisement. Les activités pédagogiques proposées par groupes interprofessionnels reposeront sur de l'analyse collective, les partage d'options et décisions, des débats d'équipes et déboucheront, en fin de journée, sur des communications restituées sur différents types de supports média. Les étudiants mobiliseront leurs connaissances sur les politiques socio-sanitaires, l'organisation des soins, l'empowerment du patient, l'exploration d'une problématique de santé, des questions éthiques et les outils du e-health. L'évaluation sera centrée sur les interactions interprofessionnelles et l'engagement dans les productions thématiques journalières. Ce type de programme est encouragé par la Confédération suisse et l'Académie suisse des sciences médicales.

Mots-clés : formation interprofessionnelle, Bachelor 
$\mathrm{CO} 33$

\section{Évaluation d'un dispositif «hybride» (e-learning/présentiel) d'évaluation par les pairs pour l'apprentissage de la pharmacovigilance, des interactions et des contre-indications des médicaments}

Hélène GÉNIAUX', Roland LAWSON², Serge BAILLY', Christelle POUGET $T^{4}$, Marie-Laure LAROCHE ${ }^{1,5}$, Nicolas PICARD ${ }^{2,6}$

${ }^{1}$ Centre régional de pharmacovigilance, de pharmacoépidémiologie et d'information sur les médicaments de Limoges, centre hospitalier universitaire (CHU) de Limoges, Limoges, France

2 Département de pharmacologie, Faculté de pharmacie, Limoges, France

3 Département CvTIC (Campus Virtuel, Technologie de l'Information et de la Communication), Faculté des sciences et techniques, Limoges, France

${ }^{4}$ Département de chimie organique et thérapeutique, Faculté de pharmacie, Limoges, France

5 Département de pharmacologie, Faculté de médecine, Limoges, France

6 Département universitaire d'enseignement numérique en santé (DUENES), Université de Limoges, Limoges, France

Contexte : La pédagogie transmissive et l'évaluation par questions à choix multiples sont très présentes en santé. L'évaluation par les pairs (EP) est une alternative qui permet de solliciter les apprenants pour des productions riches en les plaçant dans une situation de pédagogie active, susceptible de développer leur capacité à argumenter.

Objectif: Notre objectif était d'évaluer le bénéfice d'un dispositif d'EP hybride pour l'apprentissage de la pharmacovigilance, des interactions et contre-indications médicamenteuses sur deux groupes d'apprenants de l'université de Limoges, l'un en $3^{\mathrm{e}}$ année de pharmacie (diplôme de formation générale en sciences pharmaceutique : DFGSP3 filière officine, $n=36$ ) et l'autre en formation initiale ou continue de $3^{\mathrm{e}}$ cycle (diplôme d'université [DU] e-PICIM, $n=40$ ). Méthode : L'activité consistait en un «atelier» sur la plateforme d'apprentissage en ligne Moodle (version 2.9) et portait sur l'analyse de situations cliniques en rapport avec les enseignements théoriques reçus. Chaque apprenant produisait un devoir de structure définie, redistribué anonymement à 2 (DU) ou 3 (DFGSP3) de ses pairs pour évaluation selon une grille construite par les enseignants. Selon la formation (DFGSP3 ou DU), le délai accordé pour la phase de production variait de 6 à 8 jours ; celui réservé à la phase d'évaluation, de 6 à 10 jours. L'activité était suivie d'une phase présentielle: les étudiants ayant travaillé sur le même sujet présentaient, après prise en compte des EP, une synthèse orale aux enseignants. Trois notes étaient attribuées : une par les pairs, une pour l'évaluation et une de groupe, donnée par les enseignants. Á l'issue de l'activité, les apprenants ont répondu à un questionnaire d'évaluation qualitative.
Résultats : Le principe de l'EP était connu par 17,6\% des DFGSP3 et $27,5 \%$ des DU avant l'atelier et 2 étudiants (DU) avaient une expérience pédagogique antérieure utilisant cette approche. Les délais de production et d'évaluation ont été jugés «adaptés » pour plus de $94 \%$ des DFGSP3. En DU, 42,5\% des inscrits auraient souhaité plus de temps pour réaliser le devoir (15-30 jours) et $20 \%$ pour les évaluations (10-15 jours). L'EP a été jugée contraignante pour $14,7 \%$ des DFGSP3 ; ils étaient plus nombreux en DU (47,5\%). Cent pour cent des DFGSP3 (82,5\% des DU) jugent avoir tiré parti personnellement du travail d'évaluateur. La phase présentielle est apparue bénéfique pour plus de 9 étudiants sur 10 puisque $37,8 \%$ des interrogés l'ont jugée « indispensable» et $55,4 \%$ «utile». Le bénéfice global s'exprimait en termes de remise en question de son travail (79,7\% des apprenants), de développement d'un esprit critique (77\%) et d'acquisition de connaissances supplémentaires (81\%).

Conclusion : L'EP par Moodle est un dispositif bien perçu par les étudiants et bénéfique pour la formation en santé, dès le premier cycle des études. En position de correcteur/conseiller (préconisations bienveillantes), sollicitant son intelligence interpersonnelle, l'étudiant, quel que soit son niveau de formation, remet en question son propre travail, développe son esprit critique et acquiert des connaissances nouvelles. Une phase présentielle avec les enseignants contribue à optimiser l'expérience pédagogique.

Mots-clés: pharmacologie, évaluation par les pairs, Moodle, apprentissage hybride, pharmacie

\section{$\mathrm{CO} 34$}

\section{Amélioration du parcours de soin des patients atteints d'un cancer par la réalisation d'un SPOC}

\author{
Charlotte VAYSSE ${ }^{1}$, Louise MORINEAU ${ }^{2}$, Elodie \\ CHANTALAT ${ }^{1}$, Odile BEYNE-RAUZY' ${ }^{3}$, Jean-Marc \\ BACHAUD ${ }^{4}$, Fabien DESPAS ${ }^{5}$, Nathalie CAUNES- \\ HILARY $Y^{6}$, Muriel POUBLANC ${ }^{7}$, Elie SERRANO ${ }^{8}$, \\ Roland BUGAT ${ }^{9}$, Marie-Eve ROUGÉ-BUGAT ${ }^{10}$, \\ Anne-Laure FIZE ${ }^{9}$
}

${ }^{1}$ Département de chirurgie oncologique, Institut universitaire du cancer Toulouse-Oncopole, Toulouse, France

${ }^{2}$ Centre de recherche en cancérologie de Toulouse, Institut universitaire du Cancer Toulouse-Oncopole, Toulouse, France ${ }^{3}$ Département de médecine interne, Institut universitaire du cancer Toulouse-Oncopole, Toulouse, France

4 Département de radiothérapie, Institut universitaire du cancer Toulouse-Oncopole, Toulouse, France

${ }^{5}$ Département de pharmacologie, CHU Toulouse, Toulouse, France

${ }^{6}$ Département des soins de support, Institut universitaire du cancer Toulouse-Oncopole, Toulouse, France

${ }^{7}$ Département de recherche clinique, Institut universitaire du cancer Toulouse-Oncopole, Toulouse, France

${ }^{8}$ CAPTOR WP 4 research programm, Université Paul-Sabatier III, CHU Toulouse, Toulouse, France 
${ }^{9}$ CAPTOR WP 4 research programm, Institut universitaire du cancer Toulouse-Oncopole, Toulouse, France

10 Département de médecine générale, Université PaulSabatier III, Toulouse, France

Contexte: La communication entre l'hôpital et la ville, souvent fragile, nécessite d'être renforcée. Pour cela, nous avons choisi d'utiliser la pédagogie par digital-learning avec la réalisation d'un SPOC (small private online course). Cet outil n'est qu'à son balbutiement dans les facultés de médecine. À la différence d'un MOOC (massive open online course), il s'adresse à un public «ciblé ». Ce SPOC, par la formation dispensée sur le parcours de soin des patients atteints d'un cancer permettrait de fluidifier les relations entre l'hôpital et la ville.

Objectifs: Nous avons construit puis évalué ce dispositif de formation au travers la satisfaction, des caractéristiques et de la participation des apprenants.

Méthode: Ce projet collaboratif a été développé par le PHUC CAPTOR entre l'université Paul-Sabatier III, le CHU et l'institut Claudius-Regaud, choisissant une pédagogie active, collaborative, interprofessionnelle avec multiplicité des apprentissages.

Chaque session comprenait 6 semaines d'enseignement avec 6 thématiques : parcours personnalisé du patient, chirurgie des cancers, radiothérapie, traitements médicaux oncologiques, recherche clinique et soins de supports.

Pour cela, 22 experts, médicaux ou paramédicaux ont participé : 12 travaillaient dans une institution de lutte contre le cancer, 8 en ambulatoire, un médecin du réseau «Hôpital-Ville», un industriel et un institutionnel.

Ce SPOC s'adressait au public «ciblé» suivant: professionnels de santé (médicaux et paramédicaux), de l'accompagnement (assistantes sociales, membres d'une association), industries pharmaceutiques et sociétés de services aux patients. L'inscription se faisait via une plateforme permettant de recueillir les caractéristiques des apprenants.

Des vidéos sur les thématiques étaient délivrées. Des QCM, quizz, exercices collaboratifs avec apprentissage par résolution de problèmes complexes leur ont été proposés avec un forum de discussion. Cette formation offrait la possibilité de valider un DPC (Développement Professionnel Continu) et d'obtenir un certificat de réussite. La satisfaction des apprenants a été évaluée, au préalable, par un questionnaire de 29 questions validé par le groupe formation et communication et la participation par le taux de complétion, de réussite et d'engagement.

Résultats : Ce SPOC a été dispensée du 24 octobre au 31 décembre 2016 et du 27 mars au 31 mai 2017. Parmi les 1574 apprenants, 446 ont rempli un questionnaire ; la majorité était âgée de 31 à 45 ans. Il y avait 56 infirmières, 103 pharmaciens, 26 du corps médical, 53 appartenant à des associations de patients, 28 enseignants et 13 étudiants. Au total, 24,7\% travaillaient en libéral, 38,5\% dans une institution publique et $36,8 \%$ dans une institution privée.

À la fin de leur formation, $86 \%$ considéraient qu'ils avaient acquis de nouvelles connaissances, $90,8 \%$ considéraient que ce SPOC répondait à leurs attentes et $90,37 \%$ pensaient que cette formation avait un impact sur leur pratique professionnelle. Le taux de complétion était de $35,5 \%$, le taux de réussite de $71,5 \%$ et le taux d'engagement de $64,7 \%$.

Discussion : Ce SPOC est révélateur de l'intérêt positif porté à l'ensemble des thématiques concernant le parcours de soin des patients atteints d'un cancer. Ce dispositif innovant permet une large diffusion de l'information et répond à un réel besoin de formation.

Mots-clés : SPOC, cancérologie, dispositif de formation, parcours patient

\section{$\mathrm{CO} 35$}

\section{Carnet de stage informatisé : quelle évaluation par les étudiants en médecine? À propos d'une expérience en médecine d'urgence}

\section{Florian CANU, Laure DOMISSE, Bénédicte DOUAY, Paulo HENRIQUES, Carole AMSALLEM, Christine AMMIRATI}

Département de médecine d'urgence, CHU Amiens Picardie, Amiens, France

Contexte: Le second cycle des études médicales doit permettre l'acquisition de compétences cliniques et favoriser le lien entre théorie et pratique au lit du patient. Les épreuves classantes nationales (ECNi) en fin de second cycle reposent sur des dossiers progressifs (DP) avec questions à réponses multiples ( $\mathrm{QRM})$ et des QRM isolés (QI). Les étudiants semblaient avoir des difficultés à faire le lien entre le stage et les épreuves théoriques

Objectifs : Nous avons souhaité réajuster le parcours de l'étudiant en stage en médecine d'urgence et mettre en place un carnet de stage informatisé (CSi) favorisant l'auto-évaluation et le lien entre expérience en stage, savoirs théoriques et modalités d'évaluation de l'ENCi. L'objectif de ce travail est d'évaluer le ressenti de l'étudiant face à ce nouveau parcours, au carnet de stage et d'objectiver les résultats

Méthode: Un parcours en cinq phases a été établi sur 8 semaines :

- À partir d'une situation clinique vécue, chaque étudiant rédige le script clinique (SC) sur le CSi ;

- Il sélectionne le ou les item(s) correspondants de l'ECNi ;

- À partir de ce travail, l'enseignant extrait de la base informatique SIDES l'ensemble des QI correspondants ;

- L'étudiant répond par informatique ;

- La semaine suivante, l'enseignant organise une séance de synthèse et de correction. L'étudiant devait écrire un script chaque semaine (SCS1 à 8 ) et rédiger 2 à 3 cas cliniques durant son stage (CC1 à 3).

Pour évaluer ce parcours, un questionnaire de satisfaction, une autoévaluation des compétences le premier jour (M0), premier mois (M1) et fin de stage (M2) ont été réalisés ainsi que l'évaluation des réponses aux QI.

Résultats: Cinquante étudiants ont été inclus. Au total, 5541 questions issues de 750 QI rattachées à 145 items $(40 \%$ du 
programme de l'ECNi) ont été traitées avec une réussite QI de 56\% qui est supérieure au deuxième mois par rapport au début du stage $(p<0,001: 1165 / 2539(66 \%)$ au deuxième mois - 1421/3002(47\%) au premier mois). Les QI relatives à l'UE11 (urgences et défaillances viscérales aiguës), correspondantes au stage, sont mieux réussies que les autres UE ( $p=0,003: 1052 / 1700(62 \%)$ pour l'UE11 versus 2035/ $3841(53 \%)$ aux autres UE).

Le nombre de compétences déclarées «totalement maîtrisées 》 ou « maîtrisées » augmentent entre M2 et J0 ( $p=0,001$ : moyenne de 41/ 70 au M2, 22/70 à J0).

La totalité des étudiants a déclaré que le CSi a fait évoluer leurs habitudes de travail et 41/50 qu'il a répondu totalement à leurs besoins. Quatre-vingt pour cent pensent qu'il a permis de faire des liens entre clinique et connaissance théorique, physiologie et pathologie. Tous indiquaient qu'un carnet de stage informatisé sans tutorat n'avait pas d'intérêt.

Discussion et conclusion : Ces résultats montrent une progression et une adhésion des étudiants. Le CSi permet une évaluation continue et semble modifier leur façon de travailler. L'évolution future du CSi vers un système complet distanciel permettrait un gain de temps enseignant (génération et correction automatique des QI), et de proposer des contenus adaptatifs et personnalisés. Une généralisation est actuellement envisagée pour tous les stages hospitaliers.

Mots-clés: carnet de stage informatisé, étudiants en médecine, DFASM, formation pratique, e-learning

\section{4. Évaluation des apprentissages}

\section{$\mathrm{CO} 36$}

\section{Comment améliorer l'évaluation des compétences communicationnelles dans le cadre d'un ECOS sanctionnant: leçons tirées d'un symposium international}

\author{
Matteo MONTI ${ }^{1}$, Raphael BONVIN ${ }^{1}$, Christina Kloeckner \\ CRONAUER ${ }^{1}$, Soeren HUWENDIEK ${ }^{2}$ \\ ${ }^{1}$ Unité pédagogique, Faculté de biologie et médecine UNIL- \\ Lausanne, Lausanne, Suisse \\ ${ }^{2}$ Abteilungsleiter AAE, Institut für Medizinische Lehre IML, \\ Berne, Suisse
}

Contexte: La communication joue un rôle central pour les professions médicales. Elle influence l'adhérence thérapeutique et les coûts de la santé. Depuis 2011, la Suisse a un examen national de fin de formation de médecine qui évalue les compétences cliniques et communicationnelles à l'aide d'un examen clinique objectif structuré (ECOS). La communication est évaluée à l'aide d'une «grille critériée », comprenant quatre dimensions génériques $(1,2)$. Les résultats montrent une forte «cohérence interne» de ces quatre dimensions (Alpha de Cronbach 0,88 à 0,91 (3)). Cependant une faible discrimination entre les quatre items nous a incité à explorer des mesures d'amélioration (3). Malheureusement la littérature ne propose pas de modèle de référence pour une évaluation de la communication dans le contexte d'un examen sanctionnant (4).

Objectif : Ainsi nous avons organisé un symposium avec des experts suisses et internationaux afin de discuter comment les différents éléments d'un ECOS devraient être structurés afin d'évaluer au mieux les compétences communicationnelles.

Méthode: Un symposium de deux jours a été organisé en 2016, en présence de quatre experts internationaux en communication et 25 experts suisses, provenant de chacune des cinq facultés de médecine et travaillant dans les domaines de la communication ou de la pédagogie médicale. Organisés en quatre groupes de travail trois questions leurs ont été soumises :

- Est-ce que l'actuelle grille critériée est adaptée?

- La conception et le contenu des stations ECOS doivent-ils être adaptés?

- Comment améliorer la formation des évaluateurs?

Les présentations et les discussions en plénière ont été enregistrées en vidéo et étaient disponibles pour l'analyse thématique.

Résultats : Concernant la conception des stations ECOS :

- Chaque ECOS devrait inclure des stations «somatiques» incluant des défis de communication sous forme de «cues and concerns» (indices et problèmes);

- La conception de ce type de station devrait se faire en collaboration entre experts en communication et cliniciens ;

- Chaque ECOS devrait également inclure des stations avec des techniques spécifiques de communication (par ex. annonce de mauvaise nouvelle, entretien motivationnel, partage de décision);

- Un équilibre doit être cherché entre l'authenticité du jeu de rôle (qui implique une certaine liberté de réaction de la part du PS en fonction de l'attitude du candidat) et la nécessite de standardisation.

Concernant la grille d'évaluation :

- Se concentrer sur les résultats observables d'une bonne communication (par ex. « le patient est rassuré ») plutôt que sur l'adhérence à un modèle de communication spécifique;

- S'assurer qu'au-delà des critères génériques la grille d'évaluation tient compte des éventuels éléments spécifiques testés dans une station donnée;

- Concernant l'évaluation des réactions des étudiants aux cues il serait bénéfique de fournir aux examinateurs des exemples concrets de réponses «adaptées, intermédiaires et inadaptées »;

- Afin de familiariser les examinateurs et les étudiants avec le système de notation il faudrait utiliser la même grille d'évaluation tout au long des études de médecine.

Conclusion: En absence de données claires dans la littérature, le symposium international a permis de proposer des stratégies d'amélioration de l'évaluation de la communication dans le contexte d'un ECOS sanctionnant.

Mots-clés : ECOS, évaluation sanctionnante, communication 


\section{$\mathrm{CO} 37$}

\section{Intérêt de l'évaluation par les pairs pour l'apprentissage et l'évaluation des connaissances durant le premier cycle des études en sciences pharmaceutiques}

Roland LAWSON ${ }^{1}$, Hélène GÉNIAUX ${ }^{2}$, Christelle POUGET ${ }^{3}$ Jacques MONTEIL ${ }^{4,5}$, Nicolas PICARD ${ }^{1,5}$

1 Département de pharmacologie, Faculté de pharmacie, Limoges, France

${ }^{2}$ Centre régional de pharmacovigilance, de pharmacoépidémiologie et d'information sur les médicaments de Limoges, Centre hospitalier universitaire (CHU) de Limoges, Limoges, France

${ }^{3}$ Département de chimie organique et thérapeutique, Faculté de pharmacie, Limoges, France

${ }^{4}$ Département de biophysique médicale, Faculté de médecine, Limoges, France

${ }^{5}$ Département universitaire d'enseignement numérique en santé (DUENES), Université de Limoges, Limoges, France

Contexte: L'évaluation par les pairs (EP) est une approche conventionnelle pour apprécier la validité des publications ou l'originalité des projets de recherche. L'EP est également utilisée au cours des formations en ligne à grande échelle (MOOC: massive open online course). C'est également une méthode d'apprentissage reposant sur le principe du socio-constructivisme, amenée à se développer à l'université grâce aux plateformes d'apprentissage en ligne ou LMS (Learning Management System).

Objectif: Notre objectif était d'évaluer quantitativement l'intérêt de l'EP pour une formation de niveau licence en sciences pharmaceutiques. Méthode: Trente-six étudiants de l'Université de Limoges, en $3^{\mathrm{e}}$ année de pharmacie (filière officine), ont participé à l'étude. L'activité consistait en un « atelier» sur le LMS Moodle (version 2.9) et portait sur l'analyse de 8 cas d'interactions médicamenteuses rattachés à 2 sous-thématiques : pharmacocinétique (PK) $(n=4)$ et pharmacodynamie (PD) $(n=4)$. Une randomisation des étudiants en PK ou PD a été réalisée. Chaque apprenant produisait un devoir de structure définie, redistribué anonymement pour évaluation, à 3 de ses pairs ayant travaillé dans la même sous-thématique. La phase d'EP était suivie d'une phase présentielle : les étudiants ayant travaillé sur le même cas présentaient une synthèse orale aux enseignants après prise en compte des EP. Trois notes sur 20 étaient attribuées : une par les pairs, une pour l'activité de correcteur et une de groupe donnée par les enseignants. Un examen écrit anonyme de 40 QCM (20 par sous-thématique) a ensuite été réalisé. Les résultats à l'EP, à l'oral et à l'écrit ont été comparés. Les notes aux QCM des étudiants ayant bénéficié de l'EP pour l'une des sous-thématiques ont été comparées à celles des étudiants n'ayant pas bénéficié du dispositif pour cette sous-thématique.

Résultats : Les notes attribuées par les pairs s'échelonnaient de 14,6à 19,6 (médiane $=17,5)$ sans différence selon la sous-thématique. Les notes des oraux (attribuées par les enseignants) étaient également peu discriminantes $(13,6$ à 19,2; médiane $=16,7)$. Elles étaient significativement moins bonnes en $\mathrm{PD}$ qu'en $\mathrm{PK}(15,5 \pm 1,3$ vs. $16,9 \pm 2,0 ; p<0,05)$. Les notes à l'examen écrit étaient plus dispersées et non corrélées à celles de l'EP (7,7 à $16,1$; médiane $=11,4)$. Comme à l'oral, les notes aux QCM PD étaient moins bonnes que celles aux QCM PK $(10,5 \pm 2,4$ vs. $12,5 \pm 2,1$; $p<0,05$ ). Pour les étudiants ayant fait l'EP en PD, une tendance (non significative) à un bénéfice du dispositif était observée, avec des notes aux QCM PD environ $10 \%$ supérieures à celles des étudiants n'ayant pas eu d'EP dans cette sous-thématique ( $11,0 \pm 2,1$ vs. $10,0 \pm 2,7)$. Les étudiants ayant fait l'EP en PK avaient en revanche des notes aux QCM de cette sous-thématique comparables à celles des autres étudiants $(12,4 \pm 2,2$ vs. $12,7 \pm 2,1)$.

Discussion et conclusion : Dans notre étude, l'EP ne semble pas un dispositif d'évaluation des connaissances suffisamment discriminant pour se dispenser d'un contrôle individuel des connaissances. C'est en revanche un dispositif d'aide à l'apprentissage dont le bénéfice pourrait être plus important pour l'acquisition des compétences les plus complexes (interactions médicamenteuses pharmacodynamiques dans notre étude). Ces résultats nécessitent d'être confirmés sur un effectif plus important d'apprenants.

Mots-clés: pharmacologie, évaluation par les pairs, Moodle, pharmacie, contrôle des connaissances

\section{$\mathrm{CO} 38$}

\section{Dissection de l'évolution du dispositif d'évaluation en anatomie macro- et microscopique : une réponse à l'évaluation de compétences complexes dans les grands groupes} Pierre BONNET ${ }^{1}$, Vinciane CRAHAY ${ }^{2}$, Jean-Philippe
HUMBLET $^{2}$, Valérie DEFAWEUX ${ }^{1}$, Adrienne DERNIER ${ }^{1}$

${ }^{1}$ Anatomie humaine, Université de Liège, Liège, Belgique

${ }^{2}$ SMART IFRES, Université de Liège, Liège, Belgique

Contexte et problématique: L'apprentissage de l'anatomie aboutit à l'acquisition de compétences complexes. Outre la maîtrise de connaissances factuelles (noms, circuits...) l'apprenant se construit progressivement une vision interne tridimensionnelle de l'architecture du corps humain qu'il mettra en pratique au contact du corps du patient ou des représentations de celui-ci (imagerie médicale, coupes microscopiques). Le dispositif d'apprentissage mis en place entraîne ces compétences de vision spatiale par le recours à la schématisation, l'utilisation de ressources vidéo, la pratique de la dissection...

Une évaluation en phase avec le dispositif d'enseignement (alignement pédagogique) se heurte à la problématique des grands groupes : 1500 étudiants sont évalués en première année, 300 en seconde et en troisième rendant impossible le recours à une évaluation orale. L'évaluation écrite mise en place comportait dans un premier temps des questions de type QCM (connaissances factuelles) combinées à une question ouverte (QO) (réalisation d'un schéma, d'une coupe). Au départ, la correction de la $\mathrm{QO}$ était basée sur une grille de critères d'importance pondérée dont la présence ou non (mode binaire) est 
encodée dans un fichier FMP (File Maker Pro) élaboré à cet effet par l'enseignant. À chaque évaluation correspondait une fiche encodée sur ordinateur.

Description : La lourdeur de l'élaboration du fichier correctif propre à chaque question et la multiplicité des demandes de ce type de solution de correction au sein de l'institution ont permis l'élaboration d'une solution institutionnelle, le logiciel SQORE développé grâce à l'appui du Service de Méthodologie et d'Aide à la Réalisation de Test (SMART). L'étudiant est évalué sur base d'une QO rédigée dans un cadre désigné sur une feuille à lecture optique. La réponse se présente sous forme de texte, de schéma, de graphique... suivant les consignes données. En anatomie, l'énoncé comporte un ou des schémas de base afin de baliser la réponse. L'enseignant a fourni au SMART sa grille de correction établie sur base de critères divisés en indices multiples. La correction s'effectue une fois les copies scannées. Via Internet, l'enseignant accède à ses corrections. Il dispose à l'écran de la copie et de sa grille à scorer. Le dispositif est utilisé en anatomie, histologie, physiologie, psychiatrie, médecine vétérinaire, droit, Agro-Bio Tech et HEC. Une fois la correction réalisée, les résultats sont accessibles, et l'enseignant peut définir un intervalle de cotes totales dans le but de vérifier sa correction initiale (cas limites entre 9 et 11/20 par exemple).

Discussion: La dématérialisation, la traçabilité, le calcul et le transfert automatiques des notes vers le système de gestion de notes de l'institution facilitent la tâche du correcteur, mais la plus-value docimologique concerne la démarche qualité qui sous-tend l'outil, ayant pour but d'améliorer l'objectivité des corrections, notamment en imposant une grille de correction, en rendant les copies anonymes et en obligeant à corriger par question ou groupe de critères.

Conclusion: À terme le dispositif permettra le recours à des correcteurs multiples pour tout ou partie de l'évaluation permettant de s'assurer de la cohérence inter-correcteurs. À plus longue échéance, l'outil pourra être utilisé pour de l'auto-évaluation.

Mots-clés : anatomie, compétences complexes, évaluation en grands groupes, correction sur ordinateur

\section{CO 39}

\section{L'ECOS en orthophonie : exercer et évaluer les attitudes professionnelles}

\section{Trecy MARTINEZ-PEREZ, Christelle MAILLART}

Département de logopédie, Université de Liège, Liège, Belgique

Contexte : L'Examen Clinique Objectif Structuré (ECOS) est utilisé comme un outil pour exercer et évaluer les compétences cliniques des étudiants dans les formations en sciences de la santé, essentiellement en médecine (par ex. Hastie et al., 2015) et en soins infirmiers (par ex. Zhu et al., 2017). L'expérience de ce type de dispositif au niveau de la formation en logopédie/orthophonie est par contre limitée, notamment en Belgique francophone.

Dans sa forme classique, l'ECOS consiste en une consultation ou partie de consultation en présence d'un patient simulé (un malade formé, un acteur ou un enseignant), d'un étudiant et d'un évaluateur. Avant de pénétrer dans la pièce de consultation, l'étudiant reçoit quelques informations sur la consultation à réaliser. Ensuite, pendant un laps de temps déterminé, l'étudiant exécute différentes tâches cliniques avec le patient simulé.

Les compétences exercées et évaluées chez l'étudiant renvoient majoritairement à des actes techniques et du raisonnement clinique (par ex. Lukas et al., 2012; Zhu et al., 2017). Les compétences communicationnelles et relationnelles peuvent également être exercées et évaluées mais ces compétences soulèvent davantage de questions au niveau de la fiabilité des jugements réalisés par l'évaluateur (Selim et al., 2012).

Notre communication s'inscrit dans la lignée des études qui se sont plus particulièrement intéressées à l'ECOS comme outil d'évaluation des attitudes professionnelles (et pas seulement cliniques) des étudiants (Bagnasco et al., 2016; O'Sullivan et al., 2008; Saraiva et al., 2016)

Objectifs : L'objectif de notre communication est double : d'une part, partager notre expérience d'un ECOS formatif avec des étudiants en $4^{\mathrm{e}}$ année d'orthophonie à l'Université de Liège (Belgique); d'autre part, présenter les analyses de la fidélité inter-juge de la grille utilisée afin d'évaluer spécifiquement les attitudes professionnelles mobilisées par les étudiants.

Méthode: Au total, 52 étudiants de 4e année en orthophonie à l'Université de Liège (Belgique) ont participé à un ECOS formatif dans le courant de l'année académique 2016-2017. Chaque étudiant a réalisé une station de 7 minutes autour d'une vignette clinique classiquement rencontrée par les orthophonistes. Des secrétaires de l'Université de Liège ont simulé les patients et des orthophonistes ont évalué les comportements des étudiants.

Trois questionnaires ont été proposés aux étudiants : un questionnaire préalable à la réalisation de l'ECOS afin d'évaluer leur niveau d'anxiété, de motivation et leur préparation; un questionnaire complété dans la suite de l'ECOS afin de connaître leur regard sur leurs performances; un questionnaire complété trois jours après l'ECOS afin d'obtenir leur avis sur les aspects organisationnels de l'ECOS. Les réponses des étudiants sont actuellement en cours d'analyse et seront discutées lors de la communication.

Par ailleurs, 18 étudiants choisis aléatoirement ont été filmés pendant leur réalisation de l'ECOS. Actuellement, ces vidéos sont visionnées par plusieurs orthophonistes qui complètent la grille d'évaluation des compétences communicationnelles et relationnelles utilisées lors de l'ECOS (dans des conditions standardisées et identiques à celles de l'ECOS réalisé). La fidélité inter-juge sera analysée et les résultats seront discutés lors de la communication.

\section{$\mathrm{CO} 40$}

\section{Formation en réanimation néonatale : rétention des connaissances et des gestes à un an}

\section{Nathalie BESSON, Delphine RICROS}

École de sages-femmes, CHU de Bordeaux, Bordeaux, France 
Contexte et objectifs: Les sages-femmes sont souvent les professionnels de santé de première ligne pour la réanimation des nouveau-nés puisqu'en France, elles effectuent plus de la moitié des accouchements et sont présentes pour la quasi-totalité des naissances. La formation initiale des étudiants sages-femmes en réanimation néonatale est donc primordiale.

À l'école de sages-femmes de Bordeaux, la formation en réanimation néonatale est un dispositif qui se déroule de la $3^{\mathrm{e}}$ à la $5^{\mathrm{e}}$ année de formation. Ce dispositif est progressif dans l'apprentissage des gestes et des connaissances théoriques nécessaires à la réanimation néonatale.

En $3^{\mathrm{e}}$ année, la formation de réanimation est dispensée selon le modèle de l'European Resuscitation Council sur une journée qui comprend une partie théorique, des ateliers procéduraux et des mises en situation pratique de simulation basse fidélité. Cette formation est réitérée en $4^{\mathrm{e}}$ et $5^{\mathrm{e}}$ année en augmentant progressivement le nombre de gestes techniques à acquérir, l'apport théorique et en proposant des scénarios de simulation de plus en plus complexes.

Ce dispositif mobilise de nombreux professionnels de santé: pédiatres, sages-femmes et puéricultrices.

Mais ce dispositif est-il efficace? Permet-il la rétention à moyen terme des connaissances et des gestes techniques?

Méthode : Pour répondre à ces interrogations, nous avons réalisé une étude quantitative auprès des étudiants sages-femmes de $4^{\mathrm{e}}$ année pour évaluer le niveau de rétention des connaissances théoriques et des gestes techniques un an après la journée de formation.

La population étudiée comprend tous les étudiants de $4^{\mathrm{e}}$ année ayant assisté à la formation l'année précédente.

Pour la partie théorique, l'évaluation est une épreuve écrite de $10 \mathrm{~min}$ composée de 10 QCM à choix simple : 0 pour une réponse fausse et 1 pour une réponse juste (note maximale : 10 points).

Pour la partie pratique, l'évaluation est une observation directe des gestes techniques (ouverture des voies aériennes, ventilation au masque et ballon, compressions thoraciques) par un formateur à l'aide d'une grille comportant 6 items notés de 1 à 5 (note maximale: 30 points).

Pour chaque partie, la note a été convertie en pourcentage par rapport à la valeur maximale et le niveau de rétention est considéré comme satisfaisant si le score était supérieur ou égal à $80 \%$.

Résultats : Les résultats montrent, pour la partie théorique, que 22/32 étudiants ont obtenu au moins $80 \%$ de bonnes réponses. Les erreurs les plus souvent retrouvées concernent le matériel (69\%) et la posologie de l'adrénaline (53\%). Pour la partie pratique, 25/32 ont obtenu un score de $80 \%$ et plus.

La rétention est moins bonne en ce qui concerne la ventilation (tenue du masque et respect de la première séquence de ventilation).

Discussion et conclusion: Ces résultats sont en accord avec la littérature qui montre une décroissance partielle des acquisitions au fur et à mesure que l'on s'éloigne du temps de formation et la nécessité de rappels réguliers théorique et pratique. Ils montrent, en outre, l'efficacité du dispositif mis en place au sein de l'école et justifient la répétition des enseignements année après année ainsi que l'investissement des professionnels de santé dans cette formation.

Mots-clés : enseignement, sages-femmes, simulation, réanimation néonatale, évaluation
$\mathrm{CO} 41$

\section{Le perfectionnement/remédiation des médecins en exercice : une collaboration de plus de 15 ans entre un ordre professionnel et les facultés de médecine québécoises}

\author{
François GOULET, Roger LADOUCEUR, Johanne \\ THIFFAULT
}

Direction de l'amélioration de l'exercice, Collège des médecins du Québec, Montréal, Canada

Contexte et objectifs : Présenter les résultats de plus de 15 ans de collaboration entre l'Ordre professionnel des médecins (Collège des médecins du Québec) et les 4 facultés de médecine québécoises dans le but d'offrir des activités de perfectionnement/remédiation à des médecins en exercice pour la mise à jour de leurs compétences.

Méthode: Avec la collaboration des 4 facultés de médecine québécoises, le Collège des médecins du Québec (CMQ) a développé un processus qui lui permet:

- D'identifier les médecins qui nécessitent une mise à jour de leurs compétences ;

- De déterminer leurs objectifs éducatifs ;

- De proposer des interventions éducatives personnalisées ;

- D'évaluer les impacts de ces interventions éducatives.

De leur côté, les facultés de médecine doivent relever le défi d'identifier un maître de stage/tuteur pour le candidat, d'offrir des activités cliniques et éducatives dans leurs milieux cliniques, d'enseigner, de superviser et d'évaluer les médecins au terme de leurs activités éducatives.

Résultats: De 2001 à 2016, plus de 800 médecins (650 médecins de famille et 230 médecins spécialistes) ont complété un programme éducatif adapté à leurs besoins. La grande majorité était des hommes ( $81 \%)$. Trente pour cent des médecins ont effectué une intervention éducative en raison d'un retour à l'exercice après un arrêt de plus de 3 ans ou pour une modification de leur domaine d'exercice; $24 \%$ à la suite d'une plainte à la Direction des enquêtes pour un problème de qualité de soins; et $46 \%$ à la suite d'une visite d'inspection professionnelle (évaluation par les pairs de la compétence clinique). Le taux de succès des interventions éducatives était de $78 \%$. Le taux de succès variait en raison de l'âge ( $97 \%$ avant 50 ans ; $86 \%$ entre 50 et 70 ans et $62 \%$ après 70 ans).

Conclusion: Le processus éducatif développé conjointement par le CMQ et les 4 facultés de médecine québécoises est original, efficient et permet aux médecins en exercice nécessitant une mise à jour d'améliorer leurs compétences cliniques par des activités éducatives personnalisées.

Mots-clés : perfectionnement, médecins en exercice, remédiation

CO 42

Un outil didactique pour analyser les scores issus de questions à choix multiples (QCM)

\section{Eric DIONNE, Julie GRONDIN}

Faculté de médecine, Université d’Ottawa, Ottawa, Canada 
Contexte et problématique: L'évaluation des compétences en éducation médicale se fait encore aujourd'hui abondamment en mettant à profit les questions à choix multiples (QCM). Ce format d'item possède de nombreux avantages dont, entre autres, de couvrir un large éventail de contenus disciplinaires et d'être facile à corriger. Par ailleurs, ces items se révèlent être plus difficiles à bâtir qu'il n'y paraît ; pour en déterminer la valeur, leur analyse édumétrique est une opération essentielle. Ces analyses édumétriques sont parfois réalisées par des experts de la mesure, mais ce n'est pas toujours le cas. Alors que faire dans un tel cas, puisque l'étude des propriétés édumétriques des items est une étape importante du processus de validation?

Description : C'est ce dernier contexte qui nous a incités à réaliser cette recherche développement visant à créer un outil didactique s'adressant à des novices de la mesure afin de les aider à : (1) produire des analyses édumétriques s'appuyant sur la théorie classique des tests (TCT) et (2) leur offrir un accompagnement didactique afin d'aider ces apprenants novices à réaliser de façon autonome ces analyses réputées complexes. C'est dans ce contexte qu'AnDIE (Analyse didactique des items avec Excel) a été créé. AnDIE permet, non seulement, de fournir les principaux indices statistiques (ex. difficulté et discrimination d'un item, score de chacun des étudiants, intervalle de confiance, indice de Sato modifié), mais il est également conçu pour offrir une première interprétation de ces indices. L'analyste n'est donc pas laissé à lui-même dans l'interprétation, parfois rébarbative pour des non-initiés, de ces informations.

Discussion : L'approche préconisée se veut hautement didactique et l'outil se destine à des personnes qui n'ont aucune base en mesure ou en théorie des tests. Dans le cadre de cette communication, nous présenterons d'abord les fondements théoriques de ce projet de recherche développement qui s'appuient autant sur la mesure que sur la didactique. Nous ferons ensuite une brève présentation de l'outil que nous avons développé en précisant la démarche méthodologique que nous avons retenue pour y arriver. Nous conclurons en exposant les forces et les limites de ce nouvel outil didactique et les étapes que nous comptons éventuellement mettre en œuvre pour l'améliorer et le bonifier.

Mots-clés : théorie classique des tests, QCM, didactique, scores

$\mathrm{CO} 43$

\section{La certification des internes de médecine générale de Toulouse (France)}

\section{André STILLMUNKES, Emile ESCOURROU, Pierre MESTHE, Michel BISMUTH, Brigitte ESCOURROU, Stéphane OUSTRIC, Marie-Eve ROUGE-BUGAT}

Département universitaire de médecine générale, facultés de médecine, Toulouse, France

Contexte: Malgré une exigence sociétale, il n'existe pas dans le monde de modèle de certification en médecine générale.

En France, il existe un Répertoire national des certifications professionnelles (RNCP). La certification des métiers de ce répertoire repose sur une formation initiale et une évaluation sanctionnante.
Objectif : Notre objectif a été d'identifier, dans le RNCP, les métiers certifiés ayant des compétences communes avec la médecine générale, afin de pouvoir comparer leurs modalités de formation initiale et d'évaluation sanctionnante avec celles du département universitaire de médecine générale.

Méthode: Revue d'une base de données (RNCP) par deux internes de médecine générale (novembre 2015 à août 2016), avec calcul du coefficient kappa de concordance inter-observateurs.

Première étape : élimination des métiers en doublon, application des critères d'exclusion (diplômes sans finalité professionnelle, validation d'acquis d'expérience).

Deuxième étape : en comparant les compétences des métiers avec les 21 du Référentiel métier et compétences médecine générale, identification de ceux ayant plus de 2 compétences communes, puis sélection des métiers ayant des compétences communes au-delà du troisième quartile (au moins 8 compétences communes).

Troisième étape: pour les métiers sélectionnés, recueil (tableaux Word) de leurs modalités de formation (enseignement théorique, stages, portfolio, carnet de stages, tutorat), de leurs modalités d'évaluation sanctionnante (intermédiaires, finale).

Résultats: Sur les 22643 fiches du RNCP, 15 métiers ont été sélectionnés : 3 métiers ont 8 compétences communes, 3 en ont 9, 6 en ont 10, 2 en ont 11, 1 a en 13 .

Cent pour cent des métiers ont une formation théorique et des stages, $46,6 \%$ un carnet de stages, $13,3 \%$ un portfolio, $95,3 \%$ un tutorat. Cent pour cent des métiers ont des évaluations intermédiaires, $100 \%$ une évaluation finale (13\% par sommation de notes, $47 \%$ par soutenance d'un mémoire, $27 \%$ par mise en situation simulée, $13 \%$ par mise en situation réelle). Pour les métiers ayant au moins 10 compétences communes, l'évaluation sanctionnante comporte des mises en situations simulées ou réelles.

Discussion et conclusion: Au département universitaire de médecine générale :

- Les modalités de formation sont comparables à celles des 15 métiers sélectionnés ;

- Les modalités d'évaluation sanctionnante ne comportent d'évaluation intermédiaire et l'évaluation finale ne comporte pas une mise en situation simulée ou réelle, contrairement aux métiers certifiés ressemblant aux soins premiers.

Ces résultats vont guider le département universitaire de médecine générale à construire les modalités de certification des internes de médecine générale, en tant que professionnels compétents.

Mots-clés : certification, formation initiale, évaluation, médecine générale

CO 44

Élaboration d'une grille d'évaluation des stages cliniques en dentisterie

\author{
Sabine GEERTS ${ }^{1}$, Anne COLLARD ${ }^{1}$, Audrey GUÉDERS ${ }^{1}$, \\ Françoise PETERS ${ }^{2}$, Pascal DETROZ \\ ${ }^{1}$ Département de dentisterie, Liège université, Liège, Belgique
}


${ }^{2}$ Bureau pédagogique faculté de médecine, Liège université, Liège, Belgique

${ }^{3}$ Institut de formation et recherche en enseignement supérieur (IFRES), Liège université, Liège, Belgique

Contexte et problématique : Jusqu'il y a peu, l'évaluation des stages pratiques en dentisterie consistait en une appréciation globale mais personnelle de l'encadrant surveillant les étudiants dans les travaux de précliniques et les soins cliniques. Bien que tous les étudiants étaient évalués de cette façon par tous les encadrants, il va sans dire que cette façon de faire était pour le moins subjective ce qui a conduit à une profonde réflexion et à la mise en place d'un outil d'évaluation le plus objectif possible.

Le département de dentisterie de l'université de Liège avait la volonté de standardiser au mieux leur système de cotation (évaluateurs multiples) des stages cliniques ainsi qu'objectiver et énoncer clairement les critères pris en considération pour cette évaluation.

Description : Une table de spécification transversale des objectifs et des acquis d'apprentissage pendant les stages pratiques (laboratoire, préclinique et clinique) en dentisterie (depuis le Bloc 2 du Bac jusqu'au Bloc 2 du Master) a tout d'abord été réalisée. Ensuite, un référentiel de compétences à acquérir durant les stages pratiques a été rédigé.

Sur cette base, une grille d'évaluation des stages cliniques a été élaborée. Cette grille d'évaluation est fondée sur le développement de 6 compétences: comportement général (Professionnalisme); relation au patient; communication et collaboration; organisation, ergonomie et asepsie; expertise en dentisterie; coefficient de difficulté). Elle est standardisée et pondérée de manière à réduire au maximum la subjectivité associée à la notation de soins/traitements prodigués aux patients : patients non comparables à divers égards, type de soins/traitements très différents (diagnostic, techniques, difficultés...), différents enseignants cliniques évaluant les étudiants.

Ces grilles d'évaluation sont accompagnées de la partie du référentiel de compétences qui rappelle les acquis antérieurs, les apprentissages en cours et les objectifs fixés. Elles sont d'application depuis décembre 2016.

Résultats et discussion: L'utilisation de ces grilles a permis d'assurer un meilleur feedback pour l'étudiant qui sait à tout moment où en est son apprentissage (ses forces, ses faiblesses) en lien avec les compétences à développer dans le cadre des stages. Après une année académique d'utilisation, un feedback des évaluateurs ainsi que des étudiants sera récolté afin d'apporter les modifications nécessaires. Une comparaison des notes obtenues en stage lors des 2 dernières années académiques sera également réalisée afin d'identifier si cette nouvelle grille permet une évaluation plus discriminante des étudiants. Parallèlement, une table de spécification transversale des objectifs et des acquis d'apprentissage des cours théoriques tout au long du cursus sera élaborée et la cohérence de l'ensemble du programme réévaluée à la lumière des compétences à développer chez les futurs dentistes.

Mots-clés : stage, grille d'évaluation, compétences

\section{$\mathrm{CO} 45$ \\ Qu'est-ce qui motive les étudiants en médecine libanais dans leurs stages cliniques?}

\author{
Eliane AYOUB, Joanna TOHME, Hisham JABBOUR, \\ Hicham ABOU ZEID, Fadia HADDAD, Nicole \\ NACCACHE
}

Anesthésie et réanimation, Hôtel Dieu de France, Beyrouth, Liban

Contexte : La motivation des étudiants en médecine au cours de leur cursus médical, varie tellement avec les années particulièrement durant les deux dernières années d'externat, où les stages cliniques prennent une dimension considérable dans leur formation.

Objectifs: Nous avons souhaité, d'une part analyser le profil motivationnel de nos étudiants durant les 2 années d'externat et, d'autre part, étudier les différents facteurs qui pourraient susciter la motivation des externes aux stages cliniques.

Méthode : Les étudiants de $6^{\mathrm{e}}$ et $7^{\mathrm{e}}$ année de médecine, ont été soumis à un questionnaire anonyme portant sur les caractéristiques individuelles qui les ont poussé à s'engager dans les études médicales, sur l'évaluation et la détermination de leur motivation aux stages cliniques allant de la $2^{\mathrm{e}}$ à la $5^{\mathrm{e}}$ année, et enfin sur leur motivation et l'appréciation du terrain de stage de la $6^{\mathrm{e}}$ et $7^{\mathrm{e}}$ année ainsi que l'évaluation des enseignants- experts qui les encadrent au cours de leur stage.

Résultats : Sur les 130 étudiants de $6^{\mathrm{e}}$ et de $7^{\mathrm{e}}$ année, 77 étudiants ont répondu au questionnaire. Les motifs d'engagement au cours des études médicales sont fortement autodéterminés chez nos étudiants. Les facteurs qui favorisent leur motivation intrinsèque sont le choix d'entreprendre des études médicales pour cause humanitaire ou l'affinité préalable pour une spécialité chirurgicale, l'impact positif du premier contact clinique notamment le stage infirmier en $2^{\mathrm{e}}$ année, un terrain de stage diversifié, riche et varié en pathologies médicochirurgicales, ainsi que la qualité de l'encadrement clinique basé sur la réflexion.

Discussion: La motivation intrinsèque repose sur le besoin d'autonomie, de compétence et la perception de soi et de l'environnement. Au cours des études médicales, le stage clinique est une étape charnière qui suscite la motivation des étudiants en présence d'un encadrement favorisant la démarche réflexive des étudiants.

Conclusion: La motivation des externes aux stages cliniques est étroitement liée à la qualité pédagogique des stages. Cette dernière est variable et doit être une des préoccupations majeures des instances facultaires. Par ailleurs, il est important de favoriser le contact précoce avec les malades et d'adopter les principes fondamentaux basés sur l'authenticité du contexte clinique et le compagnonnage cognitif.

Mots-clés : motivation, étudiants en médecine, stages cliniques 
$\mathrm{CO} 46$

\section{Les enjeux d'une formation à et par la recherche en filières médicales professionnalisantes}

\author{
Marjan NADJAFIZADEH
}

École de sages-femmes, CHRU de Nancy, Nancy, France

Contexte: La conception des étudiants de leur apprentissage ainsi que les pratiques d'étude propres à chaque filière peuvent expliquer le type d'approche développée par l'apprenant pendant ses études. Dans la mesure où la validation d'un cursus universitaire de niveau Master passe par la réalisation d'un travail de recherche, nous nous sommes intéressés au sens donné par des étudiants en filières de médecine et de maïeutique à leur mémoire de fin d'études en tant qu'activité scientifique réflexive. L'approche souvent complexe des situations de santé nécessite non seulement l'aptitude à juger, à raisonner et à réfléchir mais aussi le développement des capacités à problématiser. Dans quelle mesure, ces étudiants qui ne semblent pas toujours bien cerner l'utilité de cet exercice peuvent-ils en saisir les fondements et en cerner les retombées sur leurs pratiques professionnelles?

Objectif: L'étude a testé si la valeur attribuée par l'étudiant à sa formation à la recherche pouvait davantage s'expliquer par sa conception initiale et son profil individuel d'apprentissage plutôt que par la spécificité du contexte pédagogique propre à sa filière d'études?

Méthode: Dans une démarche qualitative et inductive, 34 entretiens semi-dirigés ont été menés auprès de 19 étudiants sages-femmes en cinquième année et de 15 internes de gynécologie-obstétrique sur une base de volontariat. Le choix de deux collectifs d'étudiants différents s'explique d'une part par une proximité de leur champ disciplinaire, tout en offrant des différences notables en termes de variétés de situations d'apprentissages et la nature même des structures de formation. Après une retranscription intégrale, une fois anonymisé le corpus recueilli a fait l'objet d'une analyse de contenu.

Résultats : Une typologie de trois approches a été identifiée ayant permis de mieux différencier le profil d'apprentissage de ces étudiants et de comprendre leur rapport à l'apprentissage de la recherche. L'approche stratégique est la plus représentative de notre population d'étude (2/3 en médecine, $1 / 2$ en maïeutique) attribuant à la recherche, l'amélioration des pratiques professionnelles comme principal objectif. Si l'approche utilitariste est plus fréquente en maïeutique (6/19 vs. $2 / 15)$, celle qui caractérise l'individu épistémique à la recherche de sens est retrouvée de façon très marginale dans les 2 populations (4/34). Nous avons pu constater que la formation à et par la recherche a contribué à faire évoluer la conception des étudiants, bien qu'en maïeutique ils restent majoritairement attachés à l'utilité du savoir.

Conclusion : Bien que le saut qualitatif attendu à l'issue du travail de recherche ne se produit pas avec la même intensité chez tous les étudiants, un accompagnement pédagogique pertinent permettrait à l'apprenant de mieux saisir le sens de sa formation et de gagner en réflexivité. Nous posons une relation causale entre la pratique de la recherche et le développement d'une forme évoluée de la conception du savoir qui se construit au fur et à mesure de l'avancée dans l'enseignement supérieur et du passage par les différents niveaux d'apprentissage. L'acculturation à la recherche et son intériorisation relève d'un processus qui nécessite du temps.

Mots-clés : formation à la recherche, rapport au savoir, médecinemaïeutique

\section{CO 47 \\ Évaluation de l'introduction des patients enseignants dans la formation des internes de médecine générale}

\author{
Sophie HAGHIGHI ${ }^{1}$, Maria José AIRES ${ }^{2}$, Claire \\ MARCHAND ${ }^{2}$, Olivia GROSS ${ }^{2}$, Yannick RUELLE ${ }^{3}$, Cam- \\ Anh KHAU ${ }^{1}$, Alain MERCIER ${ }^{1}$, Jean-Charles VERHEYE ${ }^{2}$, \\ Remi GAGNAYRE ${ }^{2}$ \\ ${ }^{1}$ DUMG Paris 13, Paris, France \\ ${ }^{2}$ LEPS, Université Paris 13, Paris, France \\ ${ }^{3}$ Département universitaire de médecine générale, université \\ Paris 13, Bobigny, France
}

Contexte : En lien avec le développement de la démocratie sanitaire, le département universitaire de médecine générale de l'Université Paris 13 a intégré en 2014 des patients comme enseignants (PE) pour la formation des internes de médecine générale (IMG). Ils interviennent en binôme avec un médecin enseignant lors des groupes d'enseignement à la pratique réflexive entre internes (GEPRI). Ces GEPRI ont lieu 1 fois par mois, par groupe de 10 étudiants et ils permettent de discuter collectivement de situations cliniques vécues.

Objectifs : 1. Évaluer l'évolution des représentations des IMG au cours de leur formation concernant les caractéristiques et les capacités des PE ; 2. Recueillir l'opinion des IMG sur l'utilité de l'intervention des PE et leur contribution sur le développement de compétence intégrant la perspective patient.

Méthode: Un questionnaire a été spécifiquement construit à partir d'études qualitatives préalablement menées auprès des différents acteurs concernés (PE, médecin enseignant, IMG). Le questionnaire comportait 3 parties : une partie sollicitant l'opinion des IMG sur les caractéristiques (23 questions) et les capacités (11 questions) des PE (questionnaire de type PERPE sollicitant dans le même temps l'opinion qu'avaient les IMG avant la formation puis après l'avoir suivi); une partie explorant la perception d'utilité des PE; une partie évaluant la contribution du PE dans l'acquisition de compétences en lien avec la perspective patient (14 questions). Les réponses étaient recueillies selon l'échelle de Likert à 4 modalités. Le questionnaire a été administré auprès de deux promotions d'IMG $\left(2^{\mathrm{e}}\right.$ et $3^{\mathrm{e}}$ année de DES), soit 124 étudiants.

Résultats : Au total, 89,5\% (111/124) des IMG ont répondu : $51 \%$ de $2^{\mathrm{e}}$ année et $49 \%$ de $3^{\mathrm{e}}$ année. Soixante-quinze pour cent avait suivi 
entre 3 ou 4 GEPRI avec un PE. Plus de $50 \%$ des répondants étaient d'accord avec 17/23 caractéristiques et 17/20 capacités du PE avant la formation, résultat qui s'est confirmé après la formation. On note pour certaines propositions une augmentation allant de 1 à $18 \%$ d'accord après la formation. Pour 3 caractéristiques et 2 capacités des PE, les accords sont devenus majoritaires après avoir suivi la formation. Trois caractéristiques et 1 capacité n'ont pas été retenus par les IMG (plus de $50 \%$ de désaccord) après la formation. En dehors des GEPRI, l'intervention des PE sans médecin enseignant dans la formation n'est pas retenue (plus de $60 \%$ de désaccord). L'intervention des PE est jugée utile pour 9 des 11 domaines proposés (plus de $50 \%$ d'accord). Les IMG estiment avoir développé l'ensemble des compétences permettant d'intégrer la «perspective patient» dans leur pratique (entre 62 et $91 \%$ d'accord selon les compétences) et la contribution des PE est jugée élevée pour toutes ces compétences, sauf une: «la prise en compte de l'entourage lors des prises de décisions médicales ».

Conclusion : Cette étude permet de mieux caractériser l'intervention des PE dans la formation des IMG. Ces derniers reconnaissent les qualités et capacités des PE ainsi que leur utilité pour développer des compétences leur permettant de mieux intégrer «la perspective patient» dans leurs consultations.

Mots-clés : évaluation, DES de médecine générale, démocratie sanitaire, patient expert

\section{$\mathrm{CO} 48$}

\section{L'apprentissage des sciences infirmières: difficultés et remédiations}

Rachid GOUIFRANE ${ }^{1}$, Ali JAFRI ${ }^{2}$, Said BENMOKHTAR ${ }^{3}$, Lamia SLIMANI' ${ }^{2}$ Said BELAAOUAD ${ }^{3}$, Idrissa DIAWARA', Fatima DEHBI ${ }^{2}$, Mohamed RADID ${ }^{3}$

${ }^{1}$ Laboratoire chimie physique des matériaux, Faculté des sciences infirmiers et techniques de santé, Casablanca, Maroc ${ }^{2}$ Faculté des sciences infirmiers et techniques de santé, Casablanca, Maroc

${ }^{3}$ Laboratoire chimie physique des matériaux, Faculté des sciences Ben M'sik, Casablanca, Maroc

Contexte: L'apprentissage des sciences infirmières, soit en milieu académique ou en milieu clinique, est souvent jugé difficile et pénible par beaucoup d'étudiants. Certains cours sont considérés comme des cours difficiles dont la réussite demande un effort important. Les enseignants sont conscients de ces problèmes mais se renferment, souvent, dans une logique qui culpabilise l'étudiant sur la base des notes obtenues lors des examens et des évaluations. Ainsi, les problèmes liés à l'apprentissage sont rarement définis en termes de difficultés mais sont décrits comme une ignorance, un problème de niveau ou une question d'aptitude. C'est dans cette perspective que vient ce travail de recherche préalable qui vise à identifier et à décrire l'ensemble des difficultés d'apprentissage - encore peu explorées - que vivent les étudiants infirmiers. Ce diagnostic constitue, en fait, une condition primordiale pour espérer garantir un enseignement de qualité.

Objectifs : Les objectifs de la présente étude, descriptive de type mixte, étaient d'identifier les principales difficultés d'apprentissage des sciences infirmières au niveau de la faculté des sciences et techniques de santé de Casablanca, de trouver des causes explicatives et de proposer des actions de remédiation.

Méthode: L'étude s'est déroulée en deux phases. Nous avons d'abord entamé des entretiens semi structurés avec 11 encadrants: sept enseignants et quatre tuteurs de stage pour décrire les difficultés d'apprentissage observées par les encadrants. Ensuite, et sur la base des résultats obtenus, un questionnaire a été élaboré et destiné aux 54 étudiants des trois années d'étude pour explorer et estimer la fréquence des difficultés d'apprentissage perçues par ces derniers.

Résultats: L'analyse des donnés a confirmée 15 principales difficultés d'apprentissage et 21 mesures de remédiation. Les difficultés évoquées comprennent des difficultés cognitives dont les lacunes théoriques et les difficultés de raisonnement clinique, et des difficultés non cognitives: procédurales et comportementales. Les difficultés de raisonnement cliniques restent les plus fréquentes quel que soit le niveau d'étude. Pour n'en citer que les difficultés de recueil des données ( $66 \%$ des enquêtés), les difficultés à formuler et à justifier des jugements cliniques infirmiers $(72 \%)$, les difficultés à prioriser les jugements cliniques identifiés $(62 \%)$ et les difficultés d'élaboration des plan de soins ( $83 \%)$.

Discussion et conclusion: Les solutions évoquées concernent le contenu du programme, les stratégies d'enseignement, les stratégies d'évaluation et le développement personnel des étudiants. Les résultats de cette étude ouvrent plusieurs pistes de recherche, dont notre projet de digitalisation du contenu pédagogique de la deuxième année d'étude de l'option infirmier polyvalent dans le cadre d'une approche blended learning qui favorise notamment la mise en lien entre les connaissances théoriques et cliniques des étudiants.

\section{Références}

[1] Chapados C, et al. Le raisonnement clinique de l'infirmière. Rev Recherche 2014;11(1). https://www.oiiq.org/sites/default/files/uploads/ periodiques/Perspective/vol11no1/10-recherche.pdf.

[2] Charlin B. L'évaluation du raisonnement clinique. Pedagogie Médicale 2003;4:42-52. http://www.pedagogie-medicale.org/articles/ pmed/pdf/2003/01/pmed20034p42.pdf

[3] Houde C. 2014. Démarche de création d'une formation en ligne sur la pratique transfusionnelle dans le cadre d'un programme de baccalauréat en sciences infirmières en approche par compétences. Université de Montréal. https://papyrus.bib.umontreal.ca/xmlui/bit stream/handle/1866/13151/Houde_Cathy_2014_travaildirig\%C3\% A9.pdf? sequence $=4 \&$ isAllowed $=\mathrm{y}$.

Mots-clés : apprentissage, sciences infirmières, diagnostic, difficultés, remédiations 


\section{Raisonnement clinique}

CO 49

Je joue, tu joues, nous observons, nous raisonnons: développement progressif des habiletés cliniques en pratique infirmière avancée

\author{
Line LANGLOIS ${ }^{1}$, Mélanie COUPAL ${ }^{2}$, Cécile MICHAUD ${ }^{2}$ \\ 1 Département de médecine de famille, Université de \\ Sherbrooke, Sherbrooke, Canada \\ 2 École des sciences infirmières, Université de Sherbrooke, \\ Sherbrooke, Canada
}

Contexte: Au cours de la formation des infirmières en pratique avancée, le développement d'habiletés cliniques (HC) de niveau avancé occupe une place prédominante. Au Québec, chez les infirmières praticiennes spécialisées en première ligne (IPSPL), l'acquisition de ces habiletés particulièrement en lien avec l'examen physique revêt une grande importance puisque les IPSPL auront à poser des diagnostics ou appliquer des traitements qui s'appuient sur ces habiletés. Traditionnellement, tant dans la pédagogie médicale qu'en sciences infirmières, l'examen physique est enseigné sous un ordre pré établi, de la tête aux pieds. Cette approche, bien qu'utile dans certains contextes, présente plusieurs limites au niveau du raisonnement clinique et de la rétention des connaissances. Nous avons donc innové pour développer un modèle d'enseignement ainsi qu'un guide qui assure un apprentissage progressif des HC.

Objectifs :

- Créer un modèle d'enseignement novateur contribuant au développement d'HC et au développement du raisonnement clinique associé.

- Basé sur ce modèle d'enseignement, développer un guide des HC à maîtriser par les IPSPL assurant un continuum pédagogique pour les enseignants et un continuum d'apprentissage pour les étudiantes.

Méthode: Diverses approches pédagogiques dont l'apprentissage par anticipation et discrimination (Yudkowky et al., 2009; Nishigori et al., 2011), l'examen physique ciblé sur les hypothèses diagnostiques (Yudkowsky et al., 2009), le jeux de rôle et l'apprentissage en trio (Martineau et al.,2013) ainsi que l'entraînement délibéré avec rétroaction (Ericsson, 1993, 2000, 2007, 2009; Ambrose, 2010) ont été combinées pour créer le modèle d'enseignement.

Pour développer le guide des HC nous avons d'abord inventorié celles à maîtriser dans la formation en fonction de leur degré de complexité et des habiletés acquises dans la formation antérieure. Nous avons ensuite construit des canevas de base pour chaque type d'habileté qui comprend: les objectifs de la séance, la durée, le matériel et la préparation préalable (lectures, vidéos, grilles à compléter). Ces grilles comportent la séquence de l'HC en incluant une confrontation entre les hypothèses diagnostiques. La version de l'enseignant inclut, en plus, le déroulement, les jeux de rôles, de même que le corrigé des grilles.

Résultats : Des évaluations par l'entremise de groupes de discussion focalisées $(n=10)$, d'un sondage $(n=18)$ et d'entrevues individuelles $(n=2)$ auprès de professeurs et d'étudiants ont permis de recueillir quelques perceptions. Selon les professeurs, le modèle d'enseignement implanté ainsi que le guide contribuent 1) au développement du raisonnement clinique 2) à assurer la cohésion de l'apprentissage des habiletés cliniques de façon longitudinale et 3) à assurer une progression dans la complexité de l'apprentissage. Du point de vue des étudiants, les outils pédagogiques favorisent leurs apprentissages et sont utiles tout au long du parcours de formation. Les deux parties considèrent que des améliorations quant à la plateforme du guide sont nécessaires afin d'améliorer son accessibilité et rendre son utilisation plus conviviale.

Discussion : La création d'un modèle d'enseignement novateur ainsi que d'un guide pour les $\mathrm{HC}$ ont engendré des retombées pédagogiques positives tant du point de vue des professeurs que des étudiants. Les approches pédagogiques préconisées semblent améliorer le processus d'apprentissage, le développement du raisonnement clinique ainsi que le développement des HC.

Mots-clés: raisonnement clinique, hypothesis driven physical examination, examen physique ciblé sur les hypothèses diagnostiques, évaluation par les pairs, jeux de rôle, entraînement délibéré avec rétroaction, habiletés cliniques

CO 50

\section{Construction et validation d'un modèle multidimensionnel explorant le développement du raisonnement clinique chez les médecins en formation}

Hubert MAISONNEUVE ${ }^{1}$, Virginie MULLER-JUGE ${ }^{1}$, Estelle MICHELET ${ }^{2}$, Noelle JUNOD-PERROND ${ }^{1}$, Anne BARROFFIO ${ }^{3}$, Catherine JUNG ${ }^{4}$, Thierry PELACCIA ${ }^{5}$, Mathieu NENDAZ ${ }^{3}$, Marie-Claude AUDETAT ${ }^{1,3}$

${ }^{1}$ Unité des internistes généralistes et pédiatres, Faculté de médecine, Université de Genève, Genève, Suisse

${ }^{2}$ CHEM, Brest, France

${ }^{3}$ Unité de développement et recherche en éducation médicale, Faculté de médecine, Université de Genève, Genève, Suisse

${ }^{4}$ Département de médecine générale, Faculté de médecine, Université de Strasbourg, Strasbourg, France

${ }^{5}$ Centre de formation et de recherche en pédagogie des sciences de la santé, Faculté de médecine, Université de Strasbourg, Strasbourg, France

Contexte : Le raisonnement clinique $(\mathrm{RC})$ est considéré comme une compétence centrale de la pratique médicale. Appréhender sa complexité reste un challenge, tant pour les théoriciens que pour 
les superviseurs cliniques. Bien que le RC soit au cœur des préoccupations des enseignants, il existe relativement peu de littérature en ce qui concerne son développement, particulièrement lors des études médicales. De plus, aucune des théories existantes ne permet de modéliser le développement du RC dans sa globalité, ce qui explique l'absence de consensus, à ce jour, pour décrire les différentes étapes et processus d'un tel développement.

Notre recherche vise à mettre en lumière les liens théoriques et empiriques entre ces différentes théories, de façon à élaborer un modèle intégrateur susceptible de décrire la réalité et la complexité des étapes et des processus de développement du RC des médecins en formation.

Objectifs : Élaborer et valider un modèle intégrateur décrivant le niveau de développement du RC chez des médecins en formation.

Méthode: Nous avons utilisé une méthode de recherche-action participative structurée autour de cycles réflexifs. Nous avons constitué un groupe restreint (GR), composé de six chercheurs et cliniciens experts en éducation médicale, puis un groupe élargi (GE), composé du GR et de 3 experts en supervision.

Nous avons procédé par étapes: 1) recension des écrits (GR);2) construction du modèle intégrateur au cours de cycles itératifs de discussion avec confrontation entre les données de la littérature et l'expérience des participants (GR); 3) validation du contenu du modèle par le GE puis auprès d'experts du RC lors de la conférence internationale sur le RC ; 4) adaptation du modèle $(\mathrm{GR})$; 5) validation du contenu du modèle par le GE et validation d'apparence par le GE. Résultats: Notre modèle s'adresse à des médecins supervisant directement ou indirectement une entrevue clinique impliquant un étudiant en médecine ( $2^{\mathrm{e}}$ ou $3^{\mathrm{e}}$ cycle $)$.

Le modèle contient 3 dimensions : l'échelle «ORIME», la qualité du discours en fonction de l'utilisation de qualificatifs sémantiques, la notion de script.

Il contient aussi 9 descripteurs : la familiarité de l'étudiant avec la situation clinique rencontrée, la décision médicale partagée avec le patient au cours de la situation clinique rencontrée, la réflexion avant l'action, la réflexion après l'action, la démarche de genèse des hypothèses et de recueil des données, l'intégration des autres professionnels dans le RC, le niveau de connaissances factuelles de l'étudiant, l'accompagnement de l'étudiant par le superviseur, la supervision.

Une définition générale et une description de chacun des niveaux explorés, accompagnée par un exemple, sont proposés dans un mode d'emploi.

Discussion : Par sa capacité à mettre en évidence, à représenter et à expliciter les aspects fondamentaux des processus en jeu, notre modèle intégrateur permettra aux cliniciens superviseurs de mieux appréhender la complexité du RC. Il les aidera à mieux apprécier le développement du RC de leurs étudiants. Le recueil de multiples situations nous donnera une image du développement du $\mathrm{RC}$ entre les premières expériences en clinique et la fin du post-grade.

Mots-clés : raisonnement clinique, développement, supervision, modèle intégrateur
CO 51

\section{Description d'un outil innovant d'évaluation formative du raisonnement clinique chez les professionnels de santé}

\author{
Mathieu LORENZO ${ }^{1,2}$, Thierry PELACCIA ${ }^{1}$, Bernard \\ CHARLIN $^{3}$, Emmanuel TRIBY ${ }^{4}$ \\ 1 CFRPS, Faculté de médecine de Strasbourg, Strasbourg, \\ France \\ ${ }^{2}$ Département de médecine générale, Faculté de médecine de \\ Strasbourg, Strasbourg, France \\ ${ }^{3}$ CPASS, Université de Montréal, Montréal, Canada \\ 4 LISEC, Faculté des sciences de l'éducation, Université de \\ Strasbourg, Strasbourg, France
}

Contexte et problématique : La formation au raisonnement clinique chez les professionnels de santé est un défi important en éducation médicale. En utilisant des consultations simulées et les principes des formations par concordance, les auteurs ont conçu un outil informatique d'évaluation formative du raisonnement clinique.

Description : Sur le plan conceptuel, cet outil repose le modèle MOT de l'Université de Montréal, pour distinguer différentes étapes de la démarche de raisonnement. Il est demandé à l'étudiant de renseigner à chacune de ces étapes sa représentation du problème de santé du patient simulé. Un panel d'expert a été constitué afin de répondre aux mêmes questions. L'étudiant peut ainsi bénéficier d'un feedback reposant sur la comparaison de ses réponses et de leurs justifications avec celles du panel d'expert, ainsi qu'avec celles de ses pairs. Ce dispositif de formation vise à améliorer la formation des futurs professionnels de santé (1) au processus de raisonnement clinique et (2) à favoriser l'organisation des connaissances de l'étudiant selon des modèles utiles au raisonnement (scripts, prototypes et cas concrets).

Discussion : Ce dispositif est en phase d'évaluation pour en tester les qualités psychométriques.

Cette proposition de communication orale vise à présenter le dispositif et la réflexion sous-tendant sa création, ainsi que les premiers résultats relatifs à son évaluation. Elle permettra aux auditeurs de découvrir les principes de sa mise en œuvre, dans la perspective d'une utilisation future.

Mots-clés : raisonnement clinique, évaluation formative, formations par concordance, consultations simulées

CO 52

\section{Aborder le développement des savoirs et du raisonnement clinique infirmier par la perspective de la concordance des scripts}

\author{
Marie-France DESCHÊNES ${ }^{1,2}$, Johanne GOUDREAU ${ }^{1,2}$ \\ 1 Faculté des sciences infirmières, Université de Montréal, \\ Montréal, Canada
}


${ }^{2}$ Centre d'innovation en formation infirmière, Université de Montréal, Montréal, Canada

Contexte et problématique: Un des défis actuels des institutions d'enseignement est de concevoir et de planifier l'articulation des activités éducatives pour favoriser le développement optimal des savoirs et du raisonnement clinique infirmier. Car prendre des décisions cliniques dans des contextes d'incertitude et de complexité est devenu la norme de la pratique actuelle des infirmiers. Cette réalité place l'infirmier nouvellement diplômé dans des situations où il devra faire preuve d'un raisonnement clinique solide et ce, dès le début de sa carrière. Un déficit de raisonnement clinique dont l'apprentissage n'a pas été suffisamment soutenu pendant la formation initiale potentialise les risques d'erreurs et peut conséquemment porter des préjudices à la qualité et à la sécurité des soins dispensés à la population. Dès lors, il s'avère essentiel de repenser la qualité de l'accompagnement pédagogique et d'ajuster les activités éducatives entre autres, par une cohérence beaucoup plus affinée entre la formation et la pratique du raisonnement clinique infirmier.

Compétence au cœur de la pratique infirmière, le raisonnement clinique se caractérise par les processus métacognitifs et cognitifs récursifs engagés par l'infirmier [1]. En situation de pratique où des zones d'incertitude persistent, un raisonnement clinique est situé ou contextualisé. Il sollicite un savoir aiguisé où l'infirmier s'appuie sur de riches réseaux de savoirs hautement élaborés et structurés, appelés «scripts mentaux» pour résoudre des problèmes cliniques. Mais, comment les scripts peuvent-ils éclairer pertinemment le développement des savoirs infirmiers et subséquemment le développement du raisonnement clinique infirmier? Comment rendre tangibles l'explication et l'utilisation pédagogique de ces savoirs «en acte»?

Exégèse : Le but de cette revue intégrative des écrits est d'aborder la perspective des scripts mentaux face au développement des savoirs imbriqués au raisonnement clinique infirmier et de considérer ses enjeux pédagogiques.

Une conceptualisation des activités pédagogiques favorisant le développement des savoirs et du raisonnement clinique infirmier est élaborée, en s'appuyant sur la théorie des scripts mentaux [2-4]. Cette perspective théorique propose de mettre en branle de nouvelles stratégies éducatives alliant à la fois la conscience situationnelle, l'acuité réflexive et le dialogue cognitif. Elle suggère de surcroît un ancrage théorique et disciplinaire solide pour contribuer au développement de connaissances en pédagogie des sciences infirmières.

La conception théorique abordée par cette revue intégrative propose des stratégies éducatives à mettre en place et ce, en toute congruence aux processus cognitifs mobilisés et liés au raisonnement clinique infirmier en situations authentiques de la pratique professionnelle.

\section{Références}

[1] Goudreau J, Boyer L, Létourneau D. Clinical nursing reasoning in nursing practice: a cognitive learning model based on a think aloud methodology. Quality Advancement in Nursing Education/Avancées en formation infirmière 2014;1(1).

[2] Schmidt HG, Norman GR, Boshuizen HPA. A cognitive perspective on medical expertise. Theory and implications. Acad Med 1990;65(10):611-21.
[3] Charlin B, Tardif J, Boshuizen HPA. Scripts and medical diagnostic knowledge: theory and applications for clinical reasoning instruction and research. Acad Med 2000;75(2):182-90.

[4] Custers EJ. Thirty years of illness scripts: theoretical origins and practical applications. Med Teach 2015;37(5):457-62.

Mots-clés : raisonnement clinique, théorie des scripts, stratégies éducatives, revue intégrative, formation infirmière

\section{CO 53}

\section{Comment favoriser l'expression du raisonnement clinique des étudiants en médecine grâce à la méthode SNAPPS ? Description de la méthode et retour d'expérience dans un service de médecine légale}

\author{
Audrey FARRUGIA ${ }^{1,2}$, Jean Sébastien RAUL ${ }^{1}$, Thierry \\ PELACCIA ${ }^{3,4}$
}

1 Institut de médecine légale, Faculté de médecine de Strasbourg, Strasbourg, France

2 Centre de formation et de recherche en pédagogie des sciences de la santé, Faculté de médecine de Strasbourg, Strasbourg, France

${ }^{3}$ Centre de formation et de recherche en pédagogie des sciences de la santé, Faculté de médecine de Strasbourg, Strasbourg, France

4 SAMU 67, pôle d'anesthésie-réanimation chirurgicale, Hôpitaux universitaires de Strasbourg, Strasbourg, France

Contexte et problématique: La formation des étudiants en médecine doit non seulement leur permettre d'acquérir de solides connaissances scientifiques, mais surtout de mobiliser ces connaissances efficacement pour résoudre les problèmes, dans le cadre de ce que l'on nomme le raisonnement clinique. Le raisonnement clinique désigne l'activité intellectuelle selon laquelle un soignant collecte des données dans l'environnement et les confronte à ses connaissances et expériences antérieures dans le but de les utiliser pour prendre une décision diagnostique et de prise en charge.

Les temps de supervision clinique des étudiants par les cliniciensenseignants constituent des moments privilégiés pour soutenir et accompagner le développement de cette compétence chez les étudiants. Plusieurs stratégies ont été décrites dans la littérature scientifique pour aider les étudiants à développer leur raisonnement clinique. Nous avons choisi d'expérimenter la méthode SNAPPS avec des étudiants de cinquième et sixième année de médecine, en stage dans le service médecine légale de la faculté de médecine de Strasbourg. Cette approche, développée par Wolpaw et al. (2003) permet de révéler le raisonnement clinique des étudiants ainsi que leurs incertitudes, tout en respectant le temps limité dont dispose le clinicien-enseignant pour offrir une telle supervision.

Description : Les auteurs présenteront les principes de la méthode SNAPPS, ses modalités de mise en œuvre et les ajustements réalisés 
afin de la déployer dans un service de médecine légale. Cette activité innovante à la faculté de médecine de Strasbourg a été jugée très utile par les étudiants qui plébiscitent son utilisation dans d'autre stage clinique. Ils soulignent en particulier, au même titre que les cliniciensenseignants, son intérêt pour les aider à se poser les bonnes questions, pour prendre le temps d'y répondre par eux-mêmes lors d'une analyse bibliographique, et pour conforter leurs hypothèses grâce au feedback ciblé du tuteur.

Mots-clés : raisonnement clinique, incertitudes, SNAPSS

\section{CO 54}

\section{Développement et validation d'une évaluation du raisonnement clinique intégrée à une séance de simulation médicale}

\section{Thomas PENNAFORTE, Nathalie LOYE}

Département de mesure et évaluation en éducation, Université de Montréal, Montréal, Canada

Contexte: Le raisonnement clinique est une compétence centrale de l'exercice médical (Franck, 2005). Son évaluation reste cependant un défi (Ilgen et al., 2012). Parmi les tests rapportés, les problèmes de raisonnement clinique (PRC) présentent l'intérêt d'évaluer la génération d'hypothèse, l'identification et l'interprétation de nouvelles données (Groves, 2002). Les tests de concordance de scripts (TCS) sont quant à eux beaucoup plus diffusés et évaluent l'interprétation d'une nouvelle donnée en contexte d'incertitude (Charlin et al., 2000). Toutefois, aucun de ces tests ne reflète l'environnement dans lequel le médecin sera appelé à activer son raisonnement clinique, puisqu'ils sont réalisés en salle de classe. De plus, ils ne prennent pas en considération l'intuition pourtant décrite dans la théorie mixte du raisonnement clinique (Pelaccia, 2011; Evans et Stanovich, 2013). Enfin, leur validité ne repose que sur l'étude de certaines propriétés psychométriques sans s'inscrire dans une véritable démarche de validation. Face à ces limites, la simulation médicale pourrait être utilisée comme contexte d'utilisation des PRC et TCS, car elle présente l'avantage de placer les participants en immersion. Le contrôle des conditions de l'environnement pourrait également permettre d'activer les processus cognitifs réflexifs et intuitifs. Enfin, la validation d'un tel outil d'évaluation par le modèle de Kane (2006) pourrait fournir une variété d'éléments de preuve sur la qualité des interprétations des données récoltées.

Objectifs : Dans le cadre d'une recherche doctorale, les objectifs de cette communication sont de décrire (1) le développement d'une séance de simulation intégrant des questions évaluatives de type PRC et TCS au cours de la mise en situation (les performances aux PRC et TCS seront comparées à celles d'un panel d'expert comme recommandé par la littérature), en insistant sur le processus de collecte de données par un logiciel adapté, et (2) les grands principes de la validation par le modèle de Kane, en insistant sur l'opérationnalisation de la collecte de preuves soutenant l'interprétation des résultats.
Méthode: Les médecins inscrits aux programmes de formation en pédiatrie des trois universités du Québec seront exposés à 4 mises en situation simulées dont les conditions de stress pourront varier. Une variété de données (données démographiques, réponses aux items des tests, marqueurs de stress et données issues de l'enregistrement du parcours visuel) témoignera du comportement des participants dans le simulateur. Ces données seront analysées afin de soutenir les différentes inférences du processus de validation proposé par Kane et pouvoir interpréter les résultats obtenus par les candidats.

Discussion et conclusion: Les retombées attendues sont de deux natures. D'un point de vue pédagogique, ce nouvel outil d'évaluation « prêt à l'emploi » aura une visée formative afin d'identifier les failles des différents processus cognitifs impliqués dans le raisonnement clinique, et ce dans des conditions proches de la réalité. La rétroaction suivant la mise en situation simulée constituera l'élément pédagogique clef et pourrait aboutir à des stratégies de remédiations personnalisées. D'un point de vue scientifique, de nouvelles connaissances relatives à la mise en œuvre du raisonnement clinique seront produites par la possibilité de croiser les nombreuses données de différentes natures collectées pendant la simulation.

Mots-clés : raisonnement clinique, simulation médicale, validation, modèle de Kane

\section{CO 55}

\section{Test de concordance de scripts (TCS) et ancrage : format «classique» ou "générique»?}

\author{
Jean-Paul FOURNIER ${ }^{1}$, Louis SIBERT ${ }^{2}$, Paul URSMAR \\ MILLIEZ ${ }^{3}$, Joly LAURE ${ }^{4}$, Marc BRAUN ${ }^{4}$ \\ ${ }^{1}$ Département de pédagogie médicale, Faculté de médecine de \\ Nice Sophia-Antipolis, Nice, France \\ ${ }^{2}$ Faculté de médecine de Rouen, France, Rouen, France \\ ${ }^{3}$ Faculté de médecine de Caen, Caen, France \\ ${ }^{4}$ Faculté de médecine de Nancy, Nancy, France
}

Contexte: Le TCS sera prochainement utilisé pour les Épreuves Classantes Nationales informatisées (ECNi) qui clôturent les études médicales en France. Les ancrages utilisent habituellement un format «spécifique» à chaque thématique des vignettes (diagnostic, investigations, prise en charge le plus souvent). Plus récemment, un format «générique», commun aux différentes thématiques a été proposé.

Objectif : Cette étude compare les 2 formats en terme de scores et de fidélité.

Méthode: Quatre facultés de médecine ont organisé un test portant sur 3 matières communes aux 4 facultés, administré aux étudiants de $4^{\mathrm{e}}$ année (DFASM 1). Vingt-cinq items comportant chacun 1 vignette et 3 questions ont été rédigés selon les recommandations publiées. Ils ont été préparés dans un des facultés et validés par une autre. Ils ont été présentés sous les 2 formats, en ordre aléatoire. Les experts et les étudiants ont été préalablement initiés aux TCS au moyen d'un tutoriel. L'épreuve comportait donc 50 items et 150 questions. Elle a 
été administrée simultanément aux 4 facultés via la plateforme SIDES, les étudiants composant surs tablettes tactiles. Aucune question ou réponse d'experts n'a été éliminée après administration de l'épreuve. Les scores ont été ramenés à 20. Ils ont été comparés par un test de Student et corrélés par la méthode de Spearman. Les coefficients de Cronbach ont été calculés.

Résultats: Les données étaient disponibles pour 438 étudiants. Trente-huit experts des 3 disciplines concernées ont constitué le panel, chaque expert ne répondant qu'aux items de sa spécialité. Pour l'ensemble de l'épreuve, le score moyen était de 7,03 $\pm 2,00$ $(6,26 \pm 2,60$ à $7,30 \pm 1,60$ selon les facultés $)$ avec un coefficient de Cronbach de 0,90 ( 0,82 à 0,94 selon les facultés). Selon le format d'ancrage utilisé, il n'y avait de différence significative ni pour le score $(6,94 \pm 1,96$ vs. $6,99 \pm 2,10)$, ni pour le coefficient de Cronbach $(0,80$ vs. 0,02$)$. Les scores étaient très significativement corrélés $\left(\mathrm{R}^{2}=0,88, p<0,001\right)$. Les résultats étaient similaires pour chaque faculté.

Discussion : Les 2 formats d'ancrage donnent des scores similaires avec une fidélité équivalente. Le score «générique» a l'avantage d'être à la fois plus proche des bases conceptuelles du TCS (il mesure l'impact d'une information supplémentaire sur une hypothèse) et plus intuitif. Les 2 formats sont disponibles sur la base SIDES.

Mots-clés : test de concordance de scripts, ancrages

\section{Recherche}

\section{CO 56}

\section{L'intention des maîtres de stage lié à l'utilisation d'un référentiel de compétences}

\author{
Pascal DETROZ1, Dider GIET ${ }^{2}$, Valerie MASSART ${ }^{2}$, \\ Johanne HUART ${ }^{1}$ \\ ${ }^{1}$ IFRES, ULG, Liège, Belgique \\ 2 DUMG, ULG, Liège, Belgique
}

Contexte: En s'appuyant notamment sur deux référentiels internationaux: le WONCA tree et le Canmeds une équipe de travail, constituée des enseignants du département de médecine générale de notre institution, accompagnés de 20 médecins en activité, a listé les activités et tâches que devait pouvoir accomplir un clinicien compétent. Ils ont ensuite organisé ces tâches en six grandes catégories. Pour chacune d'elles, une compétence centrale a été identifiée, ainsi qu'une série de familles de situations dans lesquelles cette compétence est mobilisée. Une fois finalisé, le document a été soumis à un panel d'experts de terrain et aux étudiants pour validation afin de s'assurer que le projet soit perçu comme dynamique et mobilisant.

Le référentiel terminé, la seconde étape méthodologique a été la reconfiguration de l'enseignement pour qu'il permette l'atteinte des compétences visées. Le problème qui s'est posé à l'équipe enseignante est qu'une partie importante de la formation se fait en dehors des murs de l'université : elle se passe en de nombreux lieux de stage sous la supervision de maîtres de stage, souvent des médecins en activité ayant pour la plupart peu de lien avec la faculté. Afin d'être efficace, il est cependant nécessaire que l'ensemble des intervenants dans la formation des étudiants, y compris ces maîtres de stages, utilise le référentiel de formation comme référent aux activités d'apprentissages proposées aux étudiants.

Or, une telle utilisation ne coule pas de source.

Objectif : L'objectif de cette étude est de connaître les intentions des maîtres de stage (MdS) quant à cette utilisation. Afin de connaître les éléments prédicteurs de cette intention, nous avons fait appel à la théorie du comportement planifié (Ajzen, 1985, 1991; Ajzen et Fishbein, 2005) pour guider notre démarche. Ce modèle est réputé comme étant le plus puissant pour prédire les comportements. Il a été utilisé dans de très nombreuses études, y compris dans le domaine de la santé (Reid et Wood, 2008 ; Hardeman et al, 2002 ; Stecker et al., 2007)...

Selon cette théorie le comportement peut être directement prédit à partir d'une intention et celle-ci est déterminée par trois facteurs : (1) l'attitude envers le comportement, (2) la norme subjective et (3) le contrôle perçu.

Méthode: Conformément à la méthodologie proposée par Ajzen, nous avons dès lors conçu un questionnaire mesurant de manière directe et indirecte les divers paramètres de ce modèle (attitudes, normes sociales et contrôle perçu) sur la base de sept entretiens avec des maîtres de stage. Ce questionnaire a ensuite été proposé à la population des Maîtres de Stage.

Résultats et discussion : Nous avons obtenu 68 réponses. Après avoir effectué des régressions hiérarchiques pas à pas ascendantes, nous avons conclu qu'ensemble, les attitudes, les normes sociales et le contrôle perçu expliquaient $83 \%$ de l'intention d'utiliser le référentiel. D'autres analyses ont permis de déterminer les leviers et les freins influant directement sur cette intention. Ils seront présentés lors de notre communication.

Mots-clés : théorie du comportement planifié, compétences, maîtres de stage

CO 57

\section{La pratique de la recherche permet-elle la mise en oeuvre d'un processus de réflexion chez les internes?}

\author{
Chloe DELACOUR ${ }^{1}$, Hubert MAISONNEUVE ${ }^{2}$ \\ ${ }^{1}$ Département de médecine générale, Faculté de médecine de \\ Strasbourg, Strasbourg, France \\ ${ }^{2}$ Département de médecine générale, Université de Genève, \\ Genève, Suisse
}

Contexte: La recherche en soin primaire manque d'investigateurs. Le fait de participer à un protocole de recherche pendant ses études est un facteur favorisant la participation à un protocole de recherche au cours de son exercice. Afin de communiquer des éléments motivationnels aux internes, il nous semblait pertinent d'explorer les bénéfices qu'ils pourraient retirer en participant à une étude pendant leur stage ambulatoire. Dans un travail préliminaire, nous 
avions observé que le recueil de données par des internes en stage ambulatoire induisait une réflexion sur sa pratique. Une telle réflexion est susceptible d'apporter des avantages potentiels en termes de professionnalisme et d'apprentissages.

Objectif : L'objectif de notre travail était d'explorer les processus de réflexion d'internes de médecine générale durant leur participation à un protocole de recherche pendant leur stage ambulatoire.

Méthode : Il s'agit d'une recherche qualitative analysant selon une approche phénoménologique les mémoires de recherche rédigés par les internes participant à une étude clinique durant leur stage ambulatoire. Une analyse thématique d'après le cadre conceptuel de la réflexion défini par Nguyen et al. a été conduite de manière déductive en double codage. Une analyse qualitative complémentaire, inductive, cherchait à décrire le contexte de cette recherche.

Résultats : Notre analyse a mis en évidence des traces de réflexion dans l'ensemble des mémoires de recherche. Nous avons observé une moitié de mémoire «réflexifs» i.e. contenant la totalité des composantes de la réflexion définies par Nguyen et al. Des interactions entre les différentes composantes ont été retrouvées à des degrés variables dans tous les mémoires.

Discussion : Nous avons pu identifier dans les mémoires des traces de réflexion potentiellement spontanée et authentique. Ces documents ont cependant constitué la principale limite, apportant des éléments indirects et partiels de la pensée des étudiants.

Le protocole, la rédaction des mémoires, les conflits de valeurs générés par cette expérience, le vécu émotionnel, l'environnement de recherche et les maîtres de stage semblent avoir favorisé la réflexion des internes.

En incitant les internes à questionner leur propre pratique, cette expérience peut contribuer à la construction des compétences de ces futurs professionnels. Les maîtres de stage, en favorisant pour certains la réflexion de leurs internes, pourraient en profiter euxmêmes dans leur pratique.

Conclusion : Nos résultats plaident pour une plus grande intégration de la formation à la recherche au cours du $3^{\mathrm{e}}$ cycle de médecine générale.

Mots-clés : réflexivité, recherche, internes

\section{CO 58}

\section{Comment les étudiants en sciences infirmières utilisent-ils les outils numériques pendant les cours?}

\section{Isabelle SEBRI}

Institut de formation en soins infirmiers, Groupe hospitalier St Vincent, Strasbourg, France

Contexte : Quel enseignant ne s'est pas un jour interrogé sur ce qui se passe derrière les écrans d'ordinateur, qui se sont généralisés en cours, et l'usage qui en est fait par les étudiants?

Objectifs : L'objectif de notre travail était double :

- Identifier la nature des outils numériques (ordinateur portable, tablette électronique, téléphone portable) que les étudiants en sciences infirmières amènent et utilisent pendant le cours ;
- Documenter précisément l'usage que font les étudiants de ces outils pendant le cours.

Méthode: Nous avons mené une étude descriptive, prospective et multicentrique auprès de 1446 étudiants en soins infirmiers de la région Alsace. Les données ont été recueillies entre février et avril 2015, grâce à un questionnaire anonyme autoadministré, rempli par les étudiants à l'issue d'un cours magistral auquel ils venaient de participer.

Résultats : Quatre-vingt-dix-neuf pour cent des étudiants emmènent en cours au moins un outil numérique. Quatre-vingt-dix pour cent d'entre eux ont en leur possession un téléphone portable. Celui-ci est principalement utilisé à des fins de divertissement, en particulier pour envoyer et recevoir des messages, ainsi que pour consulter les courriels. Cinquante-deux pour cent ont un ordinateur portable. Celui-ci est essentiellement utilisé pour des tâches d'apprentissage (la prise de notes, la révision des cours et le travail sur d'autres unités d'enseignement).

Discussion et conclusion : Nous avons documenté qu'une majorité d'étudiants en sciences infirmières amènent en cours un téléphone et/ ou un ordinateur portable dont l'usage est respectivement à but de divertissement et d'apprentissage. Ces résultats sont susceptibles de guider les établissements de formation dans l'élaboration de leur politique institutionnelle en matière d'usages des outils numériques en cours.

Mots-clés : étudiants en sciences infirmières, cours magistral, outils numériques

\section{CO 59}

\section{Valeur accordée aux tâches d'enseignement par des enseignants cliniques d'une Faculté de médecine québécoise}

\author{
Annie Dubeau ${ }^{1}$, Nicolas FERNANDEZ ${ }^{2}$ \\ ${ }^{1}$ Département d'éducation et formation spécialisée, Université \\ du Québec à Montréal (UQAM), Montréal, Canada \\ 2 Centre de pédagogie appliquée aux sciences de la santé, \\ Université de Montréal, Montréal, Canada
}

Contexte : La valeur accordée à la tâche (subjective task-value) est une dimension de la motivation à l'accomplissement qui a été peu étudiée dans les recherches menées dans le domaine de la pédagogie médicale. De manière prédominante, les recherches dans le domaine se prévalent de la théorie d'autodétermination pour observer des écarts entre une motivation intrinsèque et plusieurs niveaux de motivations extrinsèques. Nous postulons que le modèle de la valeur accordée à la tâche, basée sur un cadre socio-cognitif, offre une perspective davantage nuancée sur la motivation à réaliser des tâches spécifiques.

Dans le domaine de la pédagogie médicale, une meilleure compréhension de la valeur accordée aux activités d'enseignement, un déterminant de l'engagement dans une tâche, est souhaitable puisque, par leurs résultats dans des milieux d'enseignement, les médecins sont tenus d'enseigner tout en poursuivant leur travail clinique. Cette double constitution de leur tâche place régulièrement 
les enseignants de médecine devant le choix de s'engager dans l'une des deux composantes.

Objectifs : Cette étude visait à documenter les caractéristiques de la valeur accordée aux activités d'enseignement, par des enseignants en médecine, à partir des 4 dimensions de valeur (importance, intérêt, coût, utilité) développées par Eccles et Wigfield (2002). De plus, notre étude visait à vérifier l'effet des construits de la valeur accordée sur l'engagement dans les tâches d'enseignement (i.e., la persistance/ ténacité et le temps hebdomadaire consacré aux activités d'enseignement).

Méthode: Pour ce faire, nous avons recueilli des données sur la perception de la valeur accordée aux activités d'enseignement par questionnaire auto rapporté envoyé à $n=486$ cliniciens-enseignants affiliés au campus régional de l'une des facultés de médecine de la province du Québec.

Résultats : Nos résultats, basé sur les $n=85$ réponses reçues, révèlent que les enseignants de médecine accordent une valeur élevée à leurs tâches d'enseignement. Dans l'analyse plus fine des quatre dimensions de la valeur accordée, nous constatons que les enseignants sont motivés par l'intérêt qu'ils portent pour la matière enseignée et par l'utilité qu'ils accordent à l'enseignement en regard de la profession et leur pratique clinique. Finalement, les analyses de régression confirment que les construits de la valeur accordée à la tâche ont un effet significatif sur la persévérance et le temps consacré aux tâches d'enseignement, conformément aux prévisions théoriques.

Mots-clés : motivation, enseignement clinique, étude quantitative, engagement

CO 60

\section{L'influence des pairs sur l'apprentissage en petit groupe de l'examen physique}

\author{
Bernard MARTINEAU ${ }^{1}$, Silvia Mamede STUDART \\ SOARES ${ }^{2}$, Linda BERGERON ${ }^{3}$, Henk G. SCHMIDT ${ }^{2}$ \\ 1 Département de médecine familiale et de médecine \\ d'urgence, Université de Sherbrooke, Sherbrooke, Canada \\ ${ }^{2}$ Medical education, Erasmus University Rotterdam, \\ Rotterdam, Pays-Bas \\ ${ }^{3}$ Centre de pédagogie des sciences de la santé, Université de \\ Sherbrooke, Sherbrooke, Canada
}

Contexte : Les directeurs des programmes d'externat et les étudiants eux-mêmes sont préoccupés par la maîtrise insuffisante de l'examen physique (EP) des étudiants à leur entrée en stages, alors que l'EP est un élément essentiel du diagnostic pour les médecins. La plupart des écoles de médecine utilisent des activités d'apprentissages en petits groupes pour enseigner l'EP lors de la formation pré-doctorale. On sait peu de choses sur les facteurs qui affectent l'apprentissage de l'EP dans les environnements en petits groupes, en particulier en ce qui concerne l'influence des pairs lors de ces activités.

Objectifs : Cette thèse comprend une série d'études menées auprès d'étudiants en médecine de deuxième année de l'université de Sherbrooke qui visait à vérifier si l'observation de pairs, le feedback des pairs et l'ordre de pratique influençaient l'acquisition des compétences psychomotrices nécessaires à la maîtrise de 1'EP.

Résultats : Une première étude a démontré que les étudiants ayant la possibilité d'observer un pair performant mieux que ceux qui n'ont pas eu cette possibilité $(83,9 \%$ vs. $75,9 \%, p<.001)$. Il n'y avait pas d'avantage significatif à observer plus d'un pair $(83,7 \%$ vs. $84,1 \%$, $p>.05)$. Une deuxième étude a démontré que l'observation d'une bonne performance d'un pair influence positivement l'acquisition de l'EP ( $81,1 \%$ vs. $68,3 \%, p>.002$.). L'observation d'un pair semble aider, en donnant aux étudiants une image mentale de la performance à apprendre qui est plus proche qui est plus proche de leur capacité à reproduire les gestes enseignés.

Le feedback des pairs passe par les commentaires sur la performance de leurs collègues lors des séances de formation en petits groupes. Une troisième étude a montré que les commentaires des pairs contribuent positivement à la maîtrise de l'EP $(89,5 \%$ vs. $86,2 \%, p=.02)$. Les étudiants qui reçoivent des commentaires par les pairs apprennent davantage. Cependant, cette influence, étudiée dans une étude subséquente, demeure complexe. Aucune caractéristique de la rétroaction par les pairs, que ce soit la quantité ou la spécificité, ne semble expliquer l'effet positif du feedback des pairs lors de l'apprentissage de l'EP.

Beaucoup d'étudiants étant généralement réticents à être les premiers à pratiquer dans un petit groupe, nous avons vérifié dans deux autres études si l'ordre de pratique dans un petit groupe fait la différence. Les données montrent qu'à la fin de la séance de formation, les étudiants ont une performance semblable (quel que soit l'ordre qu'ils pratiquent $[p=0,706]$ ou la quantité de feedback reçus $[p=0,096])$. Conclusion: En conclusion, il semble que la formation en petits groupes de l'EP est utile pour les étudiants, car l'apprentissage est influencé positivement par l'observation et le feedback des pairs. L'utilisation de petits groupes pour apprendre l'examen physique dans les programmes de formation en sciences de la santé est une méthode de choix, non seulement pour des raisons logistiques, mais aussi pour la contribution des pairs à la maîtrise de l'EP de leurs collègues.

Mots-clés: habiletés cliniques, examen physique, pré-doctoral, apprentissage

\section{CO 61 \\ État des lieux du niveau de stress et d'anxiété des étudiants en médecine}

Léa LANCELOT ${ }^{1}$, Sophie PELLOUX ${ }^{2}$, Gwenola DE COURVILLE ${ }^{3}$, Camille BAC ${ }^{2}$, Dominique LABOURET ${ }^{4}$, Marie VIPREY $Y^{1,5}$, Anne-Marie SCHOTT-PETHELAZ ${ }^{1,5}$

${ }^{1}$ Pôle IMER, Hospices civils de Lyon, Lyon, France

2 Faculté de médecine Lyon Est, Université Claude-Bernard Lyon 1, Lyon, France

3 École des psychologues praticiens, Lyon, France

${ }^{4}$ Institut de psychothérapie et d'enseignement en sophrologie, Lyon, France

${ }^{5}$ Laboratoire HESPER EA 7425, Université Claude-Bernard Lyon 1, Lyon, France 
Contexte : Stress et anxiété sont connus pour être corrélés à plusieurs problèmes de santé mentale incluant dépression, idées suicidaires, addictions, perte d'empathie et burn-out (Dyrbye Acad Med 2006). Les étudiants en médecine représentent une population à risque de manifester des niveaux de stress et d'anxiété importants pendant leurs études mais peu de données sont disponibles en France.

Objectif : Nous avons mené une étude transversale pour quantifier le niveau de stress et d'anxiété des étudiants en médecine de la faculté de Lyon Est.

Méthode: Une étude transversale observationnelle anonyme a été menée par auto questionnaire sur l'ensemble de la promotion des étudiants de $4^{\mathrm{e}}$ année de la Faculté de médecine de Lyon Est en 20152016. Le score de Cohen a été utilisé pour mesurer le niveau de stress et la partie anxiété de l'échelle HAD (Hospital Anxiety and Depression scale) pour mesurer le niveau d'anxiété. Les étudiants ont également été interrogés sur les représentations en santé mentale, les sources de stress, les consommations de substances psychotropes, et la faisabilité d'une étude interventionnelle (volonté de participer à des ateliers destinés à les aider à mieux gérer stress et anxiété).

Résultats : Au total, 357 étudiants ont participé à cette étude. Le score moyen de stress ressenti sur l'échelle de Cohen était de 32,7 avec un écart type de 2,55. Un score supérieur à 28 signifiant que la personne à un niveau de stress perçu important. Concernant l'anxiété, $24 \%$ des étudiants avaient une symptomatologie certaine (score de 11 et plus pour l'échelle HAD) et $23 \%$ une symptomatologie douteuse (score entre 8 et 10). Environ $73 \%$ des étudiants se sentaient stressés par les Épreuves Classantes Nationales, $44 \%$ des étudiants ont déjà pensé à arrêter leurs études et $2 \%$ des étudiants ont déjà pensé plusieurs fois au suicide. Trente-six pour cent étudiants ont exprimé le souhait de participer à des ateliers destinés à les aider à mieux gérer stress et anxiété. Discussion et conclusion : Le niveau de stress et d'anxiété est élevé chez les étudiants en médecine. Cette étude transversale incite à la mise en place d'ateliers visant à aider les étudiants en médecine à mieux gérer stress et anxiété tels que des ateliers de mindfullness et de sophrologie. Il serait intéressant d'évaluer la faisabilité et l'impact de ces différentes interventions sur le niveau de stress et d'anxiété.

Mots-clés : stress, anxiété, étude transversale, étudiants en médecine, score de Cohen, échelle HAD

\section{$\mathrm{CO} 62$}

\section{Influence d'une séquence courte de formation interprofessionnelle sur la perception stéréotypique et la connaissance d'autrui chez des étudiants en santé}

Adeline PAIGNON ${ }^{1}$, Françoise CINTER ${ }^{1}$, Pierre
BELLEMARE
${ }^{1}$ Filière soins infirmiers, Haute École de Santé, Genève, Suisse
${ }^{2}$ Filière physiothérapie, Haute École de Santé, Genève, Suisse

Contexte et objectifs : La formation interprofessionnelle fait partie d'une stratégie internationale qui vise à une amélioration de la collaboration entre les professionnels de santé afin d'optimiser la prise en soin centrée sur le patient, acteur dans les prises de décisions le concernant. Les études relèvent que cette collaboration est d'autant plus efficiente si les professionnels connaissent les rôles et les compétences de chaque membre de l'équipe soignante. Toutefois, les stéréotypes partagés par les groupes professionnels sur eux-mêmes et sur les autres ont un impact préjudiciable à cette connaissance mutuelle et donc sur la collaboration interprofessionnelle. L'implémentation de formations interprofessionnelles, dès la formation initiale et sous différentes formes, favorise les interactions et permettrait aux étudiants en santé de confronter et réajuster leurs stéréotypes et leurs connaissances sur autrui. La plupart des études investiguent et observent ces changements sur plusieurs années de formation interprofessionnelle. Nous supposons dans notre étude que ces modifications pourraient apparaître sur des séquences courtes. Ainsi, une séquence courte incluant une séquence initiale de jeu sérieux suivie d'une séquence de simulation, pourrait avoir une influence positive sur les perceptions des connaissances d'autrui et des stéréotypes des étudiants.

Méthode : Trente-huit étudiants des filières physiothérapie, nutrition et diététique, soins infirmiers et médecin ont participés à l'étude. Ils ont complété une version française du questionnaire «Student Stereotypes Rating Questionnaire» évaluant leurs perceptions/ stéréotypes sur leur profession et celles des autres, ainsi qu'un questionnaire, construit pour l'étude, d'auto- et hétéro-attribution des connaissances sur la pathologie spécifique (broncho-pneumonie chronique obstructive) étudiée lors des deux séquences de formation. Ces questionnaires ont été remplis avant la séquence de jeu sérieux et après la simulation.

Résultats : Les résultats mettent en évidence une influence positive des deux séquences de formation sur les stéréotypes attribués entre groupes professionnels en présence. De manière générale, les stéréotypes attribués à autrui sont plus positifs que ceux autoattribués. Concernant les connaissances, le score d'auto-attribution est supérieur à celui de l'hétéro-attribution. Bien que non significative, on observe des modifications entre les deux temps de mesures en hétéro-attribution.

Conclusion: Les résultats tendent à soutenir l'hypothèse selon laquelle un temps court de collaboration interprofessionnelle lors d'un dispositif de formation impliquant un jeu sérieux et une simulation, pourrait conduire à des modifications substantielles des stéréotypes et des connaissances attribuées à autrui entre les étudiants mis en présence. Ces résultats méritent d'être reproduits avec des collectifs estudiantins plus importants afin de vérifier la tendance de ces résultats et les confronter à un groupe n'ayant pas accès à un jeu sérieux préalablement à leur séquence de simulation.

Mots-clés : interprofessionalité, pratique simulée, jeu sérieux, stéréotype

\section{$\mathrm{CO} 63$}

\section{La communication médecin-patient : étude québécoise auprès des médecins en exercice}

\author{
François GOULET, Johanne THIFFAULT
}


Direction de l'amélioration de l'exercice, Collège des médecins du Québec, Montréal, Canada

Contexte : La communication médecin-patient est d'une importance capitale dans les soins de santé. Une bonne communication médecinpatient est reconnue pour avoir une influence positive sur la qualité des soins tant par la compréhension du diagnostic, l'adhérence au traitement, la résolution des symptômes et la satisfaction des patients. Objectifs : Valider un questionnaire et un processus d'évaluation de la communication médecin-patients et l'appliquer à trois groupes de médecins en exercice et à leurs patients.

Méthode : Un questionnaire comprenant 19 items, basé sur le guide du Calgary-Cambridge, a été développé et validé par Campbell et al. en 2001. Ce questionnaire a été traduit et validé, puis soumis à un échantillon de médecins québécois en exercice et à leurs patients. Les mêmes items se retrouvent tant sur le questionnaire destiné aux médecins que celui administré aux patients. Les patients devaient remplir le questionnaire immédiatement après leur consultation médicale alors que les médecins devaient remplir leur questionnaire une seule fois à la fin de leur journée de consultation.

La faisabilité a été évaluée en interviewant les assistants de recherche responsables de la cueillette des données et en analysant les réponses «non évaluable» de chacun des questionnaires. Les évidences sur la validité ont été obtenues par l'analyse factorielle. L'analyse d'items et l'analyse de la généralisabilité ont été utilisées afin d'évaluer la fiabilité de l'outil. Les médecins qui exerçaient en sans rendez-vous ont été séparés des médecins qui exerçaient avec rendez-vous. Une analyse a été faite quant à l'évaluation de la communication obtenue par les patients de celle faite par auto-évaluation de leurs médecins selon le sexe du médecin, le nombre d'années d'exercice et le type de pratique.

Résultats: L'échantillon final consistait en 91 médecins: 33 médecins de famille avec leur propre clientèle (avec rendez-vous), 27 médecins de famille exerçant en sans rendez-vous et 31 médecins spécialistes exerçant auprès de nouvelles clientèles ou auprès des patients en suivi. Au total 1224 patients ont rempli leur questionnaire. Les 3 assistants de recherche ont énoncé des difficultés à recruter les patients. Le taux de réponses «non évaluable» est à $30 \%$ pour 5 items au questionnaire. La validité interne est à 0,70 pour le alpha de Crohnback. L'analyse de généralisabilité pour le coefficient $\mathrm{G}$ est à 0,69 avec 15 patients, la moyenne des patients ayant consulté chaque médecin. Vingt-sept patients par médecin seraient le nombre raisonnable afin d'obtenir un coefficient $\mathrm{G}$ de 0,80 . Patients et médecins sont d'accord pour énoncer que la communication est moins satisfaisante lorsqu'elle se déroule au sans rendez-vous. Aucun lien significatif n'a été observé pour l'auto-évaluation des médecins selon le sexe ou leur nombre d'années d'exercice. Les médecins s'auto-évaluaient un peu plus sévèrement que leurs patients.

Conclusion: Les résultats de cette étude prouvent l'efficacité, la validité et la fiabilité de cette approche pour évaluer la communication médecin-patient.

Mots-clés : communication, médecins en exercice

\section{$\mathrm{CO} 64$}

\section{Éducation clinique guidée par les conceptions individuelles d'identité professionnelle et de bonne pratique en physiothérapie : étude phénoménologique descriptive}

M Isabel Guijarro MARTINEZ1, Ana Luisa LOPEZ VÉLEZ ${ }^{2}$, Luís Fernández ROSA ${ }^{3}$, Francisco García-Muro SAN JOSÉ ${ }^{1}$

${ }^{1}$ Kinésithérapie, faculté de médicine, Université CEU - San Pablo, Madrid, Espagne

2 Faculté d'éducation, Université du Pais Vasco, Vitoria, Espagne

${ }^{3}$ Faculté de médicine, Université CEU - San Pablo, Madrid, Espagne

Contexte: L'éducation clinique, formation que les étudiants en sciences de la santé réalisent dans des centres sanitaires, est reconnue comme un élément fondamental pour sa future pratique professionnelle. Pendant le déroulement de leur formation, les étudiants sont immergés dans le contexte réel de la profession et sont guidés dans leur apprentissage par le travail réalisé par d'autres professionnels de la santé, les tuteurs cliniques.

L'éducation clinique a fait l'objet de nombreuses recherches, mais après révision de la littérature, aucune d'entre elles ne l'ont considérée dans son ensemble comme un "phénomène» c'est-àdire, comme celle qui apparaît à la conscience du sujet tel qu'il apparaît.

Objectif : Face à cette lacune, l'objectif de cette recherche était de découvrir et de décrire le "phénomène» de l'éducation clinique en physiothérapie à partir de l'expérience des tuteurs cliniques sans la limiter par des connaissances théoriques et empiriques actuelles.

Méthode : À cet effet, on a utilisé la méthode phénoménologique descriptive d'Amedeo Giorgi basée sur la phénoménologie d'Edmund Husserl. Cette méthode nous a permis de découvrir la structure générale de l'expérience et de ses constituants ou qualités essentielles et invariantes sans lesquelles l'éducation clinique ne pourrait pas exister telle qu'elle est présente dans la conscience de chacun. Dans la recherche, ont participé sept physiothérapeutes de différentes spécialités en recueillant leur expérience grâce à des entretiens en profondeur et des journaux de bord quotidiens. Afin d'assurer la rigueur scientifique, les critères décrits par Amedeo Giorgi pour la recherche phénoménologique et la triangulation des données ont été appliqués.

Résultats : Après avoir analysé les données, 10 constituants ont été trouvés dont deux d'entre eux ont émergé avec une plus grande pertinence: (1) l'enseignement guidé par le concept d'être un bon physiothérapeute et (2) la conception de la pratique professionnelle de la physiothérapie en tant que cadre pour le développement de l'enseignement et l'apprentissage. 
Discussion: Dans le développement de l'éducation clinique on retrouve l'influence de nombreux facteurs. Dans cette recherche apparaissent les conceptions d'«identité professionnelle» et «bonne pratique» comme objectifs d'enseignement pour les tuteurs cliniques qui, comme éléments directeurs de leur activité, déterminent les résultats d'apprentissage des étudiants. L'autonomie professionnelle, le raisonnement clinique, le travail d'équipe et les meilleures attitudes sont quelques-uns des éléments à partir desquels les diverses activités sont conçues et sur lesquels l'étudiant est évalué même s'ils n'apparaissent pas explicitement dans le système d'évaluation.

Conclusion: Les conceptions de l'«identité professionnelle» et «bonne pratique» comme des éléments subjectifs et individuels peuvent être en accord ou non avec les objectifs curriculaires et supposent un défi pour le développement et la recherche de l'éducation clinique, compte tenu de la variabilité largement reconnue dans l'exercice des différentes professions de la santé et au sein de chacun d'eux. Comment les tuteurs conçoivent leur profession et son exercice? Et comment leurs conceptions sont véhiculées dans son enseignement? Ce sont les questions qu'il nous reste à répondre.

Mots-clés : éducation clinique, kinésithérapie, recherche qualitative, phénoménologie

CO 65

\section{Peut-on utiliser des étudiants-tuteurs pour la constitution des panels lors des Tests de Concordance de Scripts (TCS)?}

\section{Lucile ESPARON-MORGAN, Robin JOUAN, Jean-Paul FOURNIER, Patrick BAQUÉ}

Centre de simulation médicale, Faculté de médecine de Nice Sophia-Antipolis, Nice, France

Contexte : La constitution du panel constitue un des facteurs limitant l'utilisation du TCS en France. Récemment, on a montré que l'on pouvait utiliser des internes dans ce but.

Objectif : L'objectif de cette recherche était de vérifier la pertinence de panels constitués d'étudiants formés à l'enseignement (tuteurs).

Méthode : Nous avons vérifié la progression d'étudiants de $2^{\mathrm{e}}$ et $3^{\mathrm{e}}$ année (DFGSM 2 et 3 ) en raisonnement clinique avant et après des séances de simulation dédiées encadrées par des tuteurs de $5^{\mathrm{e}}$ et $6^{\mathrm{e}}$ année (DFASM 1 et 3 ) au moyen d'un TCS (25 vignettes, 75 questions, scores ramenés à 100). Deux panels ont été constitués : en DFGSM 1: 14 tuteurs et 14 experts; en DFGSM 2:15 tuteurs et 14 experts. Tuteurs et experts ont répondu à toutes les questions.

En post-test, toutes les questions ont été revues (coefficient de discrimination négatif, réponses «aberrantes »). La fidélité des scores a été mesurée par le score de Cronbach.

La comparaison des 2 types de panels a porté sur les scores attribués (corrélation de Pearson), la proportion de questions éliminées (coefficient de discrimination négatif, réponses «aberrantes ») par test de $\mathrm{Chi}^{2}$ avec correction de Yates, et les scores de Cronbach.
Résultats: Cent quarante-cinq étudiants de DFGSM 2, 160 de DFGSM 3 ont participé à l'étude. En DFGSM 2, 86 étudiants ont effectué le pré-test, 56 le post-test et 44 les 2 tests ; en DFGSM 3, 141 étudiants ont effectué le pré-test, 130 le post-test et 125 les 2 tests. Aucune réponse «aberrante» n'a été éliminée en pré- ou post-test. En DFGSM 2 : en pré-test : 4 et 5 questions ont été éliminées dans les panels de tuteurs et d'experts $(p=\mathrm{NS})$. Les scores de Cronbach étaient: 0,87 (tuteurs) et 0,84 (experts). Les scores étaient: $67,04 \pm 9,47$ vs. 63,95 vs. $8,27(p=0,02)$. En post-test: 4 et 7 questions ont été éliminées ( $p=\mathrm{NS})$. Les scores de Cronbach étaient : 0,80 et 0,77 . Les scores étaient: $73,58 \pm 7,16$ vs. $73,63 \pm 6,64$ $(p=\mathrm{NS})$.

En DFGSM 3 : en pré-test : 5 et 6 questions ont été éliminées dans les panels de tuteurs et d'experts $(p=\mathrm{NS})$. Les scores de Cronbach étaient: 0,81 (tuteurs) et 0,78 (experts). Les scores étaient: $55,01 \pm 4,67$ vs. $62,56 \pm 6,11(p<0,001)$. En post-test: 6 et 8 questions ont été éliminées ( $p=\mathrm{NS})$. Les scores de Cronbach étaient: 0,78 et 0,78 . Les scores étaient : $65,16 \pm 7,02$ vs. $68,73 \pm 7,79$ $(p=0,001)$.

Les scores étaient significativement corrélés : pré-test DFGSM 2 : Ro: $0,937, p<0,001$, post-test DFGSM 2: Ro: 0,94, $p<0,001$, DFGSM 3 : pré-test : Ro : $0,435, p<0,001$ et DFGSM 3 post-test : Ro: $0,921, p<0,001$.

Conclusion : Les tuteurs peuvent constituer des panels fiables de TCS.

Mots-clés : test de concordance de scripts, panel, tutorat

\section{CO 66}

\section{Attitudes, prise de conscience et obstacles vis-à-vis de la dentisterie basée sur les preuves chez les étudiants de la faculté de médecine dentaire de Casablanca}

\author{
Nadia KHLIL ${ }^{1}$, Marouane EL MACHROUHI ${ }^{2}$, Ayoub \\ Bousboula ${ }^{1}$, Jamila KISSA ${ }^{1}$, Farid BOURZGUI ${ }^{3}$ \\ ${ }^{1}$ Parodontologie, Faculté de médecine dentaire de Casablanca, \\ Casablanca, Maroc \\ 2 Faculté de médecine dentaire de Casablanca, Casablanca, \\ Maroc \\ ${ }^{3}$ Orthopédie dento-faciale, Faculté de médecine dentaire de \\ Casablanca, Casablanca, Maroc
}

Objectif : Cette étude avait pour objectif d'évaluer les attitudes, la prise de conscience et les obstacles vis-à-vis de la médecine dentaire basée sur les preuves chez les étudiants de la faculté de la médecine dentaire de Casablanca (FMDC).

Méthode: Deux cent quatre-vingt-neuf questionnaires ont été distribués aux étudiants de la FMDC, comportant 24 questions relatives à l'évaluation: des attitudes, la prise de conscience et la pratique actuelle, les ressources pour guider la pratique clinique, la compréhension des termes et les éventuelles barrières à la pratique basée sur la preuve. Les données relevées ont été analysées grâce au logiciel informatique IBM SPSS statistics version 20 . 
Résultats : Le taux de réponse est de 90,5\%. Au total, 78,2\% de l'échantillon est représenté par le sexe féminin. La moyenne d'âge est de 23,89 $\pm 2,05$ ans. La plupart des étudiants ont exprimé des attitudes positives mais une mauvaise compréhension de la pratique fondée sur des preuves. Ils pensaient, en effet, que la recherche scientifique influence la pratique quotidienne. Une littérature contradictoire, le manque de temps ainsi que la difficulté à évaluer le niveau d'une preuve représentaient les principales barrières. Les internes et résidents étaient plus conscients et avaient une meilleure compréhension par rapport aux étudiants de second cycle.

Discussion : Cette étude est la première du genre réalisée sur une population d'étudiants en médecine dentaire dans notre pays. Des stratégies éducatives s'avèrent nécessaires pour accroître la compréhension et l'utilisation de la pratique fondée sur les preuves en dentisterie. Puisse la réforme des études de médecine dentaire relever ce défi.

Mots-clés : attitudes, awareness, barriers, evidence based dentistry

CO 67

\section{Penser le stage de médecine générale en tant que dispositif d'apprentissage : analyse de la dynamique maître de stage - stagiaire et leviers pour l'implication de l'étudiant}

\section{Coralie THEYS, Sophie LECONTE, Louis VAN MAELE}

Centre académique de médecine générale, Université catholique de Louvain, Bruxelles, Belgique

Contexte et objectifs : En Belgique, les étudiants de médecine sont amenés à réaliser des stages en médecine générale. Dans le cadre d'une réforme du programme d'étude, et dans une perspective d'évaluation et d'amélioration du dispositif d'apprentissage en stage, le centre universitaire de médecine générale a souhaité comprendre et caractériser le fonctionnement des stages de médecine générale. Plus spécifiquement, l'objectif ici est d'analyser comment les interactions entre le maître de stage et le stagiaire influencent le degré d'implication de l'étudiant.

Méthode: Il s'agit d'une recherche qualitative dans laquelle nous avons réalisé des entretiens compréhensifs. Ces entretiens ont été menés avec des étudiants et des maîtres de stage qui participent aux stages de médecine générale en formation pré-graduée. Nous avons construit notre échantillonnage afin de diversifier les expériences de stage et les profils rencontrés selon la région d'exercice, le type de pratique, le niveau d'intervention dans le cursus et l'encadrement pédagogique. Ces entretiens ont été menés par salves afin de pouvoir ajuster la grille d'entretien aux nouveaux thèmes émergents, et ce, jusqu'à saturation des données. Selon l'analyse par théorisation ancrée, au fur et à mesure que des catégories conceptualisantes émergeaient, un tableau d'analyse était élaboré. Celui-ci nous a permis de comparer les éléments des différents entretiens.
Résultats : L'implication du stagiaire et la dynamique d'apprentissage entre l'étudiant et le maître de stage s'ancrent dans des dimensions organisationnelles, émotionnelles et inter-actionnelles. Ainsi, la formalisation des objectifs du stage et la coordination des apprentissages de l'étudiant, les attentes et la reconnaissance ressenties par le stagiaire, les discussions entre maître de stage et étudiant sur leur fonctionnement en binôme à l'intérieur d'une consultation sont des exemples de ces dimensions. Elles interviennent comme leviers à l'implication tout au long du stage, de l'arrivée du stagiaire à son intégration dans la consultation.

Conclusion : La dynamique entre le maître de stage et le stagiaire repose sur des éléments concrets qui peuvent être formalisés et travaillés dans le but d'impliquer davantage l'étudiant dans les activités de la consultation. De futures recherches sont nécessaires afin d'analyser si la formalisation de ces éléments auprès des stagiaires et des maîtres de stages améliore la satisfaction des acteurs et l'acquisition de compétences attendues.

Mots-clés : stage, médecine générale, dynamique d'apprentissage

\section{Simulation}

CO 68

Travail interprofessionnel pour la gestion des urgences vitales de l'enfant: de meilleurs leaders pour de meilleures équipes!

\author{
Daniel Aiham GHAZALI I,2, Stéphanie RAGOT ${ }^{3}$, Denis \\ ORIOT $^{4}$ \\ ${ }^{1}$ Urgences, CHU Bichat, Paris, France \\ 2 Centre de simulation Ilumens, Université Paris-Diderot, \\ Paris, France \\ ${ }^{3}$ CIC, Inserm 1402, Université de Poitiers, Poitiers, France \\ ${ }^{4}$ Urgences pédiatriques, CHU de Poitiers, Poitiers, France
}

Contexte : La sûreté d'un patient en urgence vitale dépend des gestes techniques et de la thérapeutique entrepris. Elle dépend également de l'algorithme de prise en charge, c'est-à-dire la séquence dans laquelle sont réalisés les traitements. Enfin, elle dépend des compétences non techniques de gestion de la situation d'urgence par une équipe interprofessionnelle menée par un leader. La simulation haute-fidélité (SHF) permet d'améliorer les performances du leader et de l'équipe. Cependant à notre connaissance, la relation entre ces deux performances n'a jamais été étudiée.

Objectifs : 1. Mesurer les performances du leader et de l'équipe en SHF. 2. Analyse de la corrélation entre les performances.

Méthode: L'étude biomédicale randomisée, prospective a été approuvée par l'ANSM et le CPP. Le protocole de recherche a été publié [1]. Elle incluait 48 participants randomisés en 12 équipes pluri-professionnelles de SMUR (Service Mobile d'Urgences et de Réanimation). Elles étaient composées d'un médecin urgentiste, d'un interne, d'une infirmière et d'un ambulancier. Les performances 
techniques et non techniques de ces équipes ont été évaluées lors d'une séance de SHF d'un état de choc hypovolémique du nourrisson à l'aide d'échelles validées et publiées. La performance technique a été évaluée à l'aide d'une échelle de pose de voie intra-osseuse (VIO) et l'échelle de performance d'équipe Team Average Performance Assessment Scale (TAPAS). La performance non-technique a été évaluée par les échelles Behavioral Assessment Tool (BAT) pour le leader et Clinical Teamwork Scale (CTS) pour l'équipe. La relation entre les différents scores de performance a été étudiée avec un coefficient de corrélation de Pearson. $p<0,05$ était considéré comme significatif.

Résultats : Les scores (normalisés sur 100) étaient: VIO $=65,6$ $\pm 14,4 ; \quad$ TAPAS $=44,6 \pm 18,1 ; \quad$ BAT $=49,5 \pm 22,0 ; \quad$ CTS $=50,3$ $\pm 18,5$. Il y avait une forte corrélation entre la performance du leader et celles de l'équipe : BAT et CTS $(r=0,962, p<0,001)$, et BAT et TAPAS $(r=0,815, p<0,001)$. Lorsque le score BAT du leader était supérieur à 60/100, ceux de l'équipe CTS et TAPAS étaient supérieurs à 50/100. Une faible corrélation a été retrouvée entre VIO et CTS $(r=0,287, p=0,04)$. Il n'y avait pas de corrélation entre BAT et VIO $(p=0,14)$ et entre TAPAS et VIO $(p=0,10)$.

Discussion et conclusion: Les performances technique et nontechnique d'équipe étaient corrélées à celles du leader. La tâche unique de pose de VIO était moins liée à la performance du leader. Ces résultats suggèreraient qu'une formation spécifique dédiée au leadership pourrait améliorer le travail d'équipe et permettrait d'envisager un bénéfice indéniable pour le patient. Les futures études pourraient envisager l'évolution des performances de leader et d'équipe lors de la répétition des simulations.

\section{Référence}

[1] Ghazali A, Ragot S, Brèque C, Guéchi Y, Boureau-Voultoury A, Petitpas F, Oriot D. Randomized controlled trial of multidisciplinary team stress and performance in immersive simulation for management of infant in shock: study protocol. Scand J Trauma Resusc Emerg Med 2016;24:36

Mots-clés : simulation, travail interprofessionnel, leader, évaluation des apprentissages

CO 69

Peut-on faire mourir un patient simulé ? À propos d'une étude portant sur l'impact de la mort d'un mannequin de haute technologie sur le sentiment d'efficacité personnelle des étudiants en médecine

\author{
Anne WEISS ${ }^{1}$, Morgan JAFFRELOT ${ }^{2}$, Thierry PELACCIA ${ }^{3}$ \\ ${ }^{1}$ SAMU/CESU, CHU Strasbourg, Strasbourg, France \\ ${ }^{2}$ CHEM, CHEM Brest, Brest, France \\ ${ }^{3}$ SAMU/MCU faculté, CHU Strasbourg, Strasbourg, France
}

Contexte : La mort du patient simulé est controversée. Pour certains, faire mourir un mannequin est préjudiciable pour l'apprentissage (Beck, 1997) ; pour d'autres, c'est une façon de préparer les étudiants à ces situations (Nickerson et al, 2009). Le sentiment d'efficacité personnelle (SEP) reflète la perception qu'a une personne de sa capacité à réaliser une tâche. C'est un moteur de l'action en situation de soin, qui se forge principalement à partir des expériences passées. Objectif : Dans cette étude, nous avons cherché à mesurer l'impact de la survenue de la mort d'un mannequin dans un environnement simulé sur le SEP des étudiants en médecine concernant leur capacité à gérer un arrêt cardiaque.

Méthode : Nous avons réalisé une étude prospective observationnelle monocentrique. Dans le groupe $1(n=27)$, des étudiants en médecine prégradués étaient prévenus, lors d'un prebriefing, de l'évolution possible de la situation simulée vers la mort du mannequin; ils ne l'étaient pas dans le groupe $2(n=29)$. Le SEP des étudiants a été mesuré à l'issue de la mise en situation simulée et après le débriefing. Résultats: Le SEP des deux groupes était similaire avant le débriefing $(p=0,41)$. Il a progressé significativement à l'issue du débriefing $(p=0,000295)$. Aucune différence significative n'a été retrouvée entre les 2 groupes $(p=0,3816)$.

Conclusion : La mort simulée du mannequin n'a pas d'impact négatif sur le SEP des étudiants, qu'ils aient ou non été prévenus de l'éventualité de survenue d'un tel événement. Notre étude contribue à alimenter la posture consistant à défendre la place de la mort inattendue du mannequin en simulation haute-fidélité.

Mots-clés: simulation, décès, éducation médicale, sentiment d'efficacité personnelle

$\mathrm{CO} 70$

\section{Débriefing en simulation : évaluer la qualité du débriefing et les compétences des formateurs}

\section{Isabelle BORRACCIA, Véronique BRUNSTEIN}

Centre d'enseignement des soins d'urgence, $C H R U$ Strasbourg, Strasbourg, France

Contexte: La simulation est une technique pédagogique d'un grand intérêt sur le plan de l'apprentissage réflexif, sous réserve d'en respecter la structure et la méthode. Le débriefing est une des étapes essentielles dont la qualité peut varier selon plusieurs facteurs. L'évaluation de la pratique du débriefing en simulation est une recommandation de l'HAS qui vise à maintenir et à améliorer les compétences des formateurs utilisant cette technique.

Objectif : Dans cette étude nous avons souhaité évaluer la qualité du débriefing en simulation, en explorant les critères ayant un impact positif ou négatif. Cette étude a été menée auprès de 20 formateurs en différenciant les formateurs experts des novices.

Question de recherche: " $\mathrm{Au}$ regard des recommandations de bonnes pratiques, de novice à expert, quelles sont les différences dans la façon de faire?».

Méthode: Nous avons réalisé cette étude en observation directe à l'aide d'une grille d'observation, testée au préalable. En parallèle, les formateurs procédaient à leur auto-évaluation à l'aide de la grille DASH (Debriefing Assessment for Simulation in Healthcare ${ }^{\circledR}$ $\left.\mathrm{DASH}^{(}\right)$ 
Le recueil des données s'est effectué sur 3 mois, pour 20 débriefings auprès de formateurs novices et experts.

Nous avons procédé à une analyse, comparative basée sur 5 thèmes (les aspects pédagogiques, les capacités de communication, la dynamique de groupe, l'aspect relationnel, les facteurs perturbateurs) Comparaison des deux groupes (experts et novices). Comparaison entre observation et auto-évaluation.

Résultats : Si on se réfère aux recommandations de bonnes pratiques, la tenue des débriefings est dans l'ensemble bien respectée par les formateurs :

Les apprenants sont impliqués, actifs, la bienveillance et le climat de confiance instaurés par les formateurs. Les 3 phases du débriefing (auto-évaluation, analyse, synthèse) sont présentes dans $96 \%$ des cas, et ce de façon égale entre les 2 groupes de formateurs.

Les objectifs du débriefing ne sont annoncés après l'auto-évaluation que dans $41 \%$ des cas. Chez les «formateurs novices» cet oubli est plus marqué puisqu'il concerne $75 \%$ d'entre eux.

Les formateurs favorisent le raisonnement clinique lors du débriefing, néanmoins certains formateurs novices utilisent plutôt des questions fermées et ont tendance à monopoliser la parole. Les temps de silence ne sont pas toujours respectés : $10 \%$ des «formateurs expérimentés 》 et $20 \%$ «des formateurs novices » limitent ainsi le raisonnement des apprenants.

À noter tout de même que certains formateurs ne s'évaluent pas toujours correctement, ce qui peut limiter leur progression et que ceux qui ont posé plus de $50 \%$ de questions fermées se sont auto-évalués sur la grille DASH à plus de 5/7 au niveau de l'item

Discussion et conclusion: Les principaux écarts entre novices et experts résident dans la capacité à questionner les apprenants et à respecter les temps de silence favorisant la réflexivité. Les autoévaluations permettent aux formateurs de cibler leurs difficultés et de mettre en évidence les discordances entre l'auto et l'hétéro-évaluation. Les résultats préliminaires puis la poursuite de cette étude permettront : - La réflexion en équipe sur un questionnement favorisant le raisonnement clinique;

- Un tutorat des formateurs novices permettra de poursuivre l'amélioration.

Mots-clés : débriefing, simulation, grille DASH

CO 71

Émotions en simulation en santé : levier ou frein pour l'apprentissage? Étude qualitative phénoménologique par entretiens semi-dirigés

Amélie GATIN ${ }^{1,2}$, Mathilde HAUET ${ }^{2}$, Marc BRAUN ${ }^{1}$, Claude VAROQUI ${ }^{1}$, Jean Michel KLEFFERT ${ }^{1}$, Hind HANI ${ }^{1}$, Isabelle LACREUSE-TALON ${ }^{3}$, Thierry PELACCIA ${ }^{3}$, Elisabeth STEYER ${ }^{4}$

${ }^{1}$ Cuesim, Université de Lorraine, Nancy, France

${ }^{2}$ Urgences pédiatriques, CHRU Nancy, Nancy, France
${ }^{3}$ UNISIMES, Université de Strasbourg, Strasbourg, France

${ }^{4}$ Département de médecine générale, Université de Lorraine, Nancy, France

Contexte et objectifs : Dans l'éducation en santé, la simulation haute fidélité est une méthode pédagogique innovante où les émotions des apprenants interfèrent avec leurs performances. Les enseignants doivent apprendre à moduler ces émotions pour qu'elles soient bénéfiques à l'apprentissage. Le lien entre émotion et apprentissage a fait l'objet de nombreuses études dans le domaine des sciences de l'éducation. Mais quelles émotions sont générées lors d'une séance de simulation en santé et quels sont leurs impacts sur les apprentissages? Méthode: Nous avons réalisé une étude qualitative phénoménologique monocentrique par entretiens semi-dirigés auprès d'apprenants de séances d'enseignement par simulation haute fidélité au centre de simulation universitaire de Nancy (CUESim) inclus selon une grille de diversité préétablie. Nous avons recherché le type d'émotions provoquées, leurs causes, les stratégies d'adaptation qu'elles génèrent et leurs répercussions ressenties sur les apprentissages. Dix-sept entretiens ont été réalisés, à l'issue de la séance, sur la base du volontariat et après signature d'un consentement éclairé.

Résultats : Parmi les émotions exprimés par les apprenants, les émotions négatives: peur, crainte, appréhension ou anxiété sont prépondérantes et considérées comme mobilisatrices et nécessaires à l'apprentissage. Rarement, elles sont rapportées comme source de débordement psychologique lorsque l'intensité devient trop élevée ou que la valence de ses émotions est extrême comme pour la honte, l'impuissance ou la détresse. Les émotions positives telles que la joie, la fierté, l'anticipation sont moins longuement mentionnées dans les récits. Elles sont souvent consolidées par les enseignants et contribuent au renforcement du sentiment d'auto-efficacité personnel décrit par les apprenants comme un apport important de la simulation. Mais à intensité ou valence positive extrêmes, elles sont parfois responsables d'une absence de prise de conscience des lacunes de performance. Nous décrivons une «zone émotionnelle efficace» optimale pour les apprentissages.

Discussion et conclusion : Les émotions négatives comme positives semblent être un moteur pour l'apprentissage en simulation à condition que le point de rupture ne soit pas dépassé. Cette acmé émotionnelle a une grande variabilité individuelle.

Un outil évaluant, avant la séance de simulation, la capacité de résilience d'un apprenant face aux stresseurs d'une situation simulée, pourrait aider l'enseignant à moduler les déterminants émotionnels dont il a la maîtrise.

Mots-clés : éducation en santé, simulation en santé, apprentissage, émotion, psychologie, étude qualitative

\section{$\mathrm{CO} 72$}

\section{Développer un centre de simulation en} santé: un outil partagé, le kit "SimUcenter»

\author{
Christine AMMIRATI ${ }^{1,2}$, Béatrice JAMAULT ${ }^{3}$, Carole \\ AMSALLEM ${ }^{3}$
}


${ }^{1}$ SimUSanté, Amiens, Amiens, France

2 Centre de recherche en psychologie, Université PicardieJules-Verne, Amiens, France

3 SimUSanté, CHU-Amiens PIcardie, Université PicardieJules-Verne, Amiens, France

Description : Notre centre de simulation hospitalo-universitaire est un espace d'apprentissage innovant partagé par tous les acteurs en santé, de la formation initiale à la formation continue : professionnels, patients et aidants.

Au cœur d'un pôle santé regroupant hôpital universitaire et campus des sciences de la santé, $4000 \mathrm{~m}^{2}$ abritent des équipements pédagogiques de haute technologie et des environnements de travail fidèlement reconstitués : domicile, officine, hôpital. Les 51 espaces de simulation (dont 43 salles reliées à des régies vidéos) sont répartis sur 3 étages avec des salles d'entraînement gestuel, des salles multimédias et des environnements contextualisés parmi lesquels une cellule sanitaire d'hélicoptère, une chaîne de décontamination NRBC et un véhicule pour désincarcération. Un véhicule SimUmobile, permet de décentraliser les formations in-situ.

En un an d'existence, 7086 personnes ont bénéficié d'une formation en santé dont 5304 en formation initiale et 1782 en formation tout au long de la vie. Plus d'une centaine de formations sont aujourd'hui proposées et des champs d'expériences pédagogiques de développent avec d'autres composantes de l'université. Ayant reçu de nombreuses visites d'équipes nationales et internationales en recherche de renseignements, l'équipe de pilotage de ce centre propose de mettre à disposition sur la plateforme Web du centre un «kit SimUcenter», libre d'accès pour échanger sur les questions techniques (équipements, matériels biomédicaux, simulateurs, vidéo...), architecturales (dimensions de salles, conception de locaux spécifiques...), organisationnelles (ressources humaines, répartition des enseignements...), financières (calcul de devis...) que tout centre en construction doit résoudre. Souhaitant partager une expérience de quatre ans de réflexions, l'objectif est de promouvoir les activités pédagogiques innovantes en santé en synergie en facilitant leur mise en place et en facilitant les échanges.

Mots-clés : simulation, centre, outil, développement

\section{$\mathrm{CO} 73$}

\section{L'alliance pédagogique superviseur- stagiaire/résident: pierre angulaire de la supervision clinique}

\section{Luc CÔTÉ1, Dominique BOUCHER ${ }^{2}$, Élisabeth DÉRY ${ }^{3}$,} Jean-François ROUX ${ }^{4}$

1 Département de médecine familiale, Université Laval, Québec, Canada

2 Département de réadaptation (ergothérapie), Université Laval, Québec, Canada

3 Département de réadaptation (orthophonie), Université Laval, Québec, Canada

${ }^{4}$ Département de réadaptation (physiothérapie), Université Laval, Québec, Canada
Contexte et objectifs: Par analogie avec l'alliance thérapeutique dans le travail clinique, l'alliance pédagogique est au cœur de la supervision clinique. En effet, la qualité du lien (relation pédagogique) influence notamment l'ouverture du stagiaire/résident à la rétroaction du superviseur et la confiance du superviseur à l'égard du stagiaire/résident, et ce, peu importe la discipline clinique. De même, l'habileté à développer et maintenir cette alliance figure parmi les responsabilités du clinicien-enseignant, qui plus est lorsque cette alliance se trouve fragilisée, voire rupturée.

Bien que les écrits sur l'alliance pédagogique soient nombreux dans le domaine de la supervision en counseling et de la psychologie, on en retrouve peu en sciences de la santé. Comment les superviseurs et les stagiaires/résidents en sciences de la santé se représentent-ils l'alliance pédagogique en supervision clinique? Quels sont les facteurs facilitants et les obstacles à une bonne alliance pédagogique? Et que font les superviseurs et les stagiaires/résidents lorsqu'il y a «bris» d'alliance?

Méthode : Des réponses à ces questions seront présentées à partir des données issues d'une étude qualitative réalisée à l'été et à l'automne 2016 sur ce sujet. Des superviseurs $(n=45)$ et des stagiaires / résidents $(n=29)$ provenant de quatre programmes en sciences de la santé à l'université Laval (Québec, Canada) - médecine familiale, ergothérapie, physiothérapie et orthophonie - ont participé à des entrevues individuelles semi-dirigées, enregistrées audio. Une analyse thématique de contenu a été faite selon un processus itératif reposant sur la triangulation des chercheurs.

Résultats : Les principaux résultats seront présentés ainsi que leurs retombées pédagogiques en contexte clinique.

Mots-clés : supervision clinique, alliance pédagogique, relation superviseur-stagiaire, résident

\section{$\mathrm{CO} 74$}

\section{Simulation de gestion de conflits professionnels: leçons apprises}

\author{
Arnaud DEMOUSTIER ${ }^{1}$, Vincent Jetté POMERLEAU ${ }^{2}$, \\ Karine PHILIBERT ${ }^{1}$, Caroline BOIS ${ }^{1}$ \\ 1 Sciences infirmières, Université de Sherbrooke, Montréal, \\ Canada \\ ${ }^{2}$ Résident en psychiatrie, Université McGill, Montréal, Canada
}

Contexte : La collaboration professionnelle est une pierre angulaire de l'excellence des soins et de la satisfaction des professionnels de la santé (OMS, 2010). L'un de ses fondements, savoir gérer les conflits, est une compétence attendue des professionnels de la santé, voire une exigence de certains ordres professionnels. Si la pertinence de cette compétence est affirmée, les dispositifs de formation à privilégier ne sont pas pour autant bien identifiés. Toutefois, la simulation utilisée en formation en santé, pourrait y contribuer. Dans un contexte où les ressources sont limitées, adopter des stratégies de développement réfléchies pourrait optimiser les retombées des simulations.

Objectifs: Développer et évaluer une activité de simulation de gestion de conflits en contexte professionnel. 
Méthode : Développer la simulation avec patients standardisés (pt) (niveau 3 selon Alinier, 2007) au fil de deux cohortes en sciences infirmières $(n=302)$ en 4 phases : 1$)$ consultation d'étudiants avec permis de pratique, au sujet de situations de conflits vécues au travail, 2) élaboration des stations, 3) évaluation de la pertinence de l'activité et des ressources proposées, 4) consolidation et déploiement auprès d'une autre clientèle. Suite à une formation sur la praxéologie (3 heures), l'activité propose trois stations pour expérimenter un conflit avec un préposé expérimenté mais négligent, une physiothérapeute désabusée du manque de collaboration interprofessionnelle ou une infirmière auxiliaire surchargée tenant des propos racistes. Chaque station comprend 12 minutes d'échanges pendant lesquelles 6-8 apprenants en équipe peuvent s'alterner pour offrir la répartie au pt puis 8 minutes de rétroaction par le pt. S'ensuivent un retour avec questionnaire et une discussion en grand groupe ( $n=30 ; 30$ minutes). La pertinence perçue de l'activité et des outils proposés, les comportements à privilégier et ceux à éviter sont évalués avec des questions de type Likert (1: pas $\mathrm{du}$ tout d'accord, 7 : tout à fait en accord) et ouvertes. Statistiques descriptives et analyse thématique par comparaison et contraste effectuées (Burnard, 1997).

Résultats : Appréciée dès son lancement, l'activité s'est bonifiée par des ajouts significatifs: formation en ligne pré-activité sur la praxéologie, accès à deux aide-mémoires lors des stations et introduction d'un étudiant comme pt dans l'une des stations. Près de $90 \%$ des répondants de la $2^{\mathrm{e}}$ cohorte rapportent que cette activité les a préparés à gérer un futur conflit professionnel (Likert : $24 \%$ [5], $22,8 \%$ [6], 42,1\% [7]). La majorité reconnait la pertinence des situations. Parmi les stations, celle impliquant l'infirmière génère davantage d'émotivité. Les moins satisfaits invoquent un manque d'organisation, un temps alloué trop court, un manque de réalisme ou d'entraînement préalable. Plus de $70 \%$ mentionnent avoir bénéficié d'une ou plusieurs notions théoriques dont les modèles de praxéologie de St-Arnaud (2003), de leadership ou du message affirmatif comme outil de communication structurée.

Discussion et conclusion: Des pistes pour développer une simulation pertinente sur la gestion de conflits se précisent. Une préparation théorique antérieure est essentielle pour bénéficier de l'expérience. Les simulations avec pt offrent un environnement stimulant pour développer des compétences de gestion de conflits.

Mots-clés : simulation, gestion de conflits, collaboration interprofessionnelle

\section{CO 75}

\section{Implantation de la formation à la collaboration interprofessionnelle dans le cursus médical}

\author{
Touria ABOUSSAOUIRA ${ }^{1}$, Patrick KALASON ${ }^{2}, \quad$ K. \\ ELHATTABI ${ }^{3}$, F.Z. BENSARDI ${ }^{3}$, Meriem ESSAIDI ${ }^{4}$ \\ 1 Faculté de médecine et de pharmacie de Casablanca, \\ Casablanca, Maroc \\ K.
}

${ }^{2}$ Université Mohamed 6 des sciences de la santé, Casablanca, Maroc

${ }^{3}$ Université Hassan II, Faculté de médecine et de pharmacie de Casablanca, service des urgences chirurgicales, CHU Ibn Rochd Casablanca, Casablanca, Maroc

4 École Nationale de Commerce et de Gestion (ENCG), Casablanca, Maroc

Contexte et problématique : La compétence de collaboration a été identifiée comme l'une des compétences transversales à acquérir en milieu professionnel qui structure le fonctionnement des équipes de ces milieux. Le Référentiel de compétences en matière d'inter professionnalisme (CPIS, 2010) présente six principaux domaines à la pratique des soins: Communication interprofessionnelle, Soins centrés sur le patient et ses proches, Clarification des rôles, Leadership collaboratif, Travail d'équipe et Résolution de conflits interprofessionnels. Le développement de la compétence de collaboration devient, de ce fait, essentiel chez les étudiants. Elle permet de construire des pratiques de soins optimales et d'améliorer la qualité de leurs interactions avec les patients.

La formation et l'exercice de la pratique collaborative exigent actuellement des normes orientant le développement de ces standards de qualité (AFISS, 2011).

Description: Ce travail propose un canevas de formation à la collaboration interprofessionnelle: approche, outils et modalités pédagogiques favorables à l'apprentissage interprofessionnel dont un module en ligne, communauté virtuelle d'apprenants, atelier de discussions interprofessionnelles, projet d'équipe, simulation, stage clinique et activité réflexive. Les modalités d'évaluation proposées pour cet apprentissage sont les grilles d'auto-évaluation, écrits réflexives et $1^{\prime}$ évaluation $360^{\circ}$ qui sont combinées pour fournir à l'apprenant une meilleure rétroaction de leurs acquis en pratique collaborative et au partenariat de soins.

\section{Références}

[1] Pelaccia T. 2016. Comment former et évaluer les étudiants en médecine et en sciences de la santé. De Boeck Superieur: Louvain-laNeuve.

[2] Rossler KL, Kimble LP. Capturing readiness to learn and collaboration as explored with an interprofessional simulation scenario. Nurse Educ Today 2016;36:348-536.

\section{CO 76}

\section{Redéfinition du compagnonnage en chirurgie par l'usage de la simulation de patients numériques}

\section{Marie Reine BOUDAREL}

Mines Nancy-Campus Artem, Nancy, France

Contexte: L'émergence des technologies informatiques et numériques au service de la pédagogie, les supports multimédias (cours assistés par ordinateur, web, vidéo $2 \mathrm{D}, 3 \mathrm{D}$ et $4 \mathrm{D}$ ) et les outils 
collaboratifs distants facilitent l'acquisition des connaissances devenues disponibles de façon nomade et directement mobilisables en ligne. Ces outils invitent à reconstruire des ingénieries pédagogiques innovantes et hybrides faisant évoluer le rôle de l'enseignant et celui de l'apprenant.

Dans le domaine de l'enseignement en santé la formation initiale et continue des professionnels concernés est devenue complexe ces dernières années du fait de nombreux facteurs :

- le développement des actes ciblés et efficients (par exemple de la chirurgie ambulatoire) nécessitant un savoir-faire, une performance et une certification reconnue ;

- la complexité des nouvelles techniques/technologies (cœlioscopie, endovalvulaire, robotique, etc.), caractérisée par une nouvelle distanciation entre le patient et l'opérateur nécessitant une adaptation aux nouveaux environnements professionnels et relationnels (exemple: une ré-coordination visuelle et gestuelle difficile à enseigner sur site);

- la loi HAS «plus jamais la première fois sur le patient » qui intègre la nécessité d'une mise en pratique avant d'exercer un nouveau geste sur le malade.

Plus spécifiquement, la question de la formation continue pour les chirurgiens nécessite de répondre à une contrainte temporelle forte dans la mesure où dans cette profession aux ressources rares, les apprenants disposent de peu de disponibilités pour suivre des formations.

La combinaison de ces contraintes invite à proposer l'intégration des outils numériques dans la formation dans le but d'augmenter les connaissances et les compétences.

Objectifs : Il nous a semblé pertinent d'analyser, en utilisant les méthodes de recherche-action, un nouveau dispositif formatif mis en œuvre au sein du DU de chirurgie endovalvulaire proposé par l'École de Chirurgie de Nancy. Le propos de recherche consiste à tenter d'évaluer l'impact de l'intégration des outils numériques et de voir dans quelle mesure ils peuvent permettre d'augmenter la qualité de la pratique tout en améliorant les modalités formatives.

Méthode: L'observation s'est déroulée entre février 2016 et novembre 2016, durée totale de la formation pour un groupe d'apprenants.

Résultats : Notre propos sera d'expliciter les éléments d'évolution de ce diplôme qui préexistait et dont l'ingénierie pédagogique a été reconçue. Nous présenterons les modalités de recherche-action concernant les dispositifs mis en œuvre: enseignement à distance, utilisation d'une plateforme collaborative, utilisation de simulateurs, séances de présentiel pour en venir aux résultats perçus pour les enseignants et pour les apprenants de cette nouvelle forme de pédagogie combinant toutes les modalités : transmissif, études de cas, travaux pratiques virtuels et réels, pédagogie inversée, formation par les pairs. Nous en arriverons ainsi à qualifier ce que nous pouvons appeler un triple compagnonnage : numérique, cognitif et relationnel issu de la combinaison de toutes les modalités dans la temporalité impartie à la formation.

\section{$\mathrm{CO} 77$ \\ Nouveau concours informatisé : une expérience novatrice pour le Cambodge}

\author{
Julien ARON ${ }^{1}$, Pascal MILLET ${ }^{2}$, Thomas FASSIER ${ }^{3}$ \\ ${ }^{1}$ Faculty of Medicine, University of Health Sciences, Phnom \\ Penh, Cambodge \\ ${ }^{2}$ CHU de Bordeaux, Université de Bordeaux, Bordeaux, France \\ ${ }^{3}$ UDREM, Unité de développement et recherche en éducation \\ médical, Université de Genève, Genève, Suisse
}

Contexte et problématique: L'amélioration de la qualité des examens est un enjeu majeur (Norcini et al., 2011). L'informatisation confère des avantages techniques, mais ne garantit pas la qualité docimologique. L'objectif de cette communication est de décrire le nouveau concours de l'internat au Cambodge, organisé par l'université des sciences de santé (University of Health Sciences, $U H S)$.

Description : Le concours de l'internat existe au Cambodge depuis 1996, comme en France avant les Épreuves Classantes Nationales (ECN). Il s'agissait d'une épreuve de 2 questions rédactionnelles et 1 orale, sur 45 items sans tableau de spécification («blueprint»). En 2014, l'UHS a initié une réforme pour améliorer sa qualité docimologique. (1) Un groupe de travail a été nommé (disciplines concernées et ministères de tutelle). (2) Un tableau de spécification a été élaboré $(5$ objectifs $\times 100$ items, adaptés par consensus à partir des ECN) et transmis aux étudiants. (3) Le format de questions Single-Best Answer (SBA) a été choisi (Schuwirth et van der Vleuten, 2003). (4) Des ateliers de rédaction de QCMs ont été organisés, avec check-list qualité et relecture par les paires. (5) Les techniciens informaticiens ont été formés à Moodle.

Résultats: Le concours d'admission 2014 visait à sélectionner 131 parmi 464 candidats. Il a associé une épreuve informatisée de 80 questions en 100 minutes, suivie d'un oral de 10 minutes (questions tirées au sort, selon le blueprint) pour les candidats admissibles (score supérieur à la moyenne). Le classement a été établi sur la note pondérant écrit et oral $(70 \% / 30 \%$ respectivement):

- Critères qualité (d'après Downing et Yudkowsky, 2009; Norcini et al., 2011);

- Forces (1) Validité de contenu: utilisation du blueprint, standardisation au format SBA. (2) Fiabilité: cohérence interne (Cronbach $\alpha$ 0,86). (3) Faisabilité : coût contrôlé. (4) Acceptabilité : épreuves d'entraînement, anonymat (reconnaissance digitale) et correction instantanée très appréciés. (5) «Effet catalyseur (catalytic effect)» : perception améliorée de la responsabilité sociale de l'UHS ;

- Faiblesses (1) Validité : limitée par contraintes logistiques (130 postes informatiques, épreuve sur un jour). (2) Fiabilité : absence d'évaluation des notes orales; examen en langue étrangère. (3) Acceptabilité : plaintes sur Facebook mettant à risque le processus. 
(4) «Effet d'apprentissage (learning effect)»: tendance au bachotage limité aux items inscrits au programme.

Conclusion: L'informatisation du concours de l'internat au Cambodge permet d'initier un processus d'évaluation et d'amélioration de la qualité des examens qui se poursuit actuellement. En 2016, l'oral a été supprimé et l'épreuve rallongée. Les enjeux techniques actuels sont l'augmentation du nombre de postes informatiques et le rallongement de l'épreuve. Les coopérations internationales restent essentielles pour la formation des auteurs et l'élargissement de la banque de questions.

\section{Références}

[1] Downing SM, Yudkowsky R. 2009. Assessment in Health Professions Education (1st ed.). New York: Routledge.

[2] Norcini J et al. Criteria for good assessment: consensus statement and recommendations from the Ottawa 2010 Conference. Med Teach 2011;33(3):206-214.

[3] Schuwirth LW, van der Vleuten CP. ABC of learning and teaching in medicine: written assessment. BMJ 2003;326(7390):643-645.

Mots-clés : évaluation, aide au développement, relations internationales

\section{$\mathrm{CO} 78$}

\section{Une «chambre des erreurs" virtuelle pour réactiver les vigilances}

Joris MULLER, Céline HERNANDEZ, Béatrice TURCAN,
Gilles NOEPPEL, Dominique SCHMITT, Thierry LAVIGNE

CHRU de Strasbourg, Strasbourg, France

Contexte et problématique : La «chambre des erreurs » est un outil de simulation en santé permettant de prendre conscience de dangers souvent négligés. Cependant, sa mise en œuvre est un frein à son utilisation dans nos environnements de travail contraints par l'espace et le temps: il faut immobiliser un lieu (chambre, salle de soin, local...), disposer de personnel pour l'animer et demander aux apprenants de se déplacer. De plus, il est difficile de suivre l'évolution d'un apprenant dans le temps sur différentes situations. Notre objectif était de proposer une «chambre des erreurs» virtuelle, facilement accessible par tous, répondant aux mêmes finalités pédagogiques qu'une «chambre des erreurs» réelle.

Solution proposée/description : Avec l'aide d'une société spécialisée dans le développement de jeux sérieux, nous avons développé un logiciel permettant d'observer et d'interagir dans un environnement de santé réaliste (chambre, bloc opératoire, zone de soin...) basé sur des photographies $360^{\circ}$. Après un écran de briefing expliquant la situation, le jeu plonge l'apprenant dans un environnement de soin qui lui est familier. Il doit observer attentivement afin de rechercher des erreurs (ex. : risque infectieux, identitovigilance, pharmacovigilance...). La prise en main est simple afin de la rendre accessible à tous. Pour chaque élément interactif dans la scène, deux choix sont proposés : adapté ou non. Selon le choix de l'utilisateur, un message pédagogique apparaît. Une fois qu'il pense avoir trouvé l'ensemble des erreurs, un écran de débriefing fait apparaître tous les éléments interactifs de la scène avec les résultats. Lors d'une autre session de jeu, l'apprenant retrouvera ses anciens scores afin de pouvoir apprécier son évolution.

Les sessions de jeu sont prévues pour être courtes afin de laisser la possibilité de répéter l'expérience sur une autre scène ou d'échanger avec d'autres personnes pour renforcer les apprentissages. Le logiciel peut être utilisé sur différents supports : casque de réalité virtuelle, smartphone, tablette tactile ou simplement un ordinateur avec un navigateur internet.

Le formateur dispose d'une interface afin de suivre l'évolution des apprenants et peut ainsi proposer un parcours pédagogique adapté. Les différentes scènes sont personnalisables afin de recréer l'environnement de travail et les erreurs souhaitées par le formateur. Conclusion : Notre outil rend vise à rendre la « chambre des erreurs » accessible et peut avoir de multiples usages: formation initiale, formation continue et évaluation des connaissances. 